\title{
ON THE INELASTIC 2-SOLITON COLLISION FOR GKDV EQUATIONS WITH GENERAL NONLINEARITY
}

\author{
CLAUDiO MUÑOZ C.
}

\begin{abstract}
We study the problem of 2-soliton collision for the generalized Korteweg-de Vries equations, completing some recent works of Y. Martel and F. Merle 24, 25. We classify the nonlinearities for which collisions are elastic or inelastic. Our main result states that in the case of small solitons, with one soliton smaller than the other one, the unique nonlinearities allowing a perfectly elastic collision are precisely the integrable cases, namely the quadratic (KdV), cubic $(\mathrm{mKdV})$ and Gardner nonlinearities.
\end{abstract}

\section{Introduction and Main Results}

In this work we consider the generalized Korteweg-de Vries equation (gKdV) on the real line

$$
u_{t}+\left(u_{x x}+f(u)\right)_{x}=0, \quad \text { in } \mathbb{R}_{t} \times \mathbb{R}_{x} .
$$

Here $u=u(t, x)$ is a real-valued function, and $f: \mathbb{R} \rightarrow \mathbb{R}$ a nonlinear function, often refered as the nonlinearity of (11). This equation represents a mathematical generalization of the Korteweg-de Vries equation $(\mathrm{KdV})$, namely the case $f(s)=s^{2}$,

$$
u_{t}+\left(u_{x x}+u^{2}\right)_{x}=0, \quad \text { in } \mathbb{R}_{t} \times \mathbb{R}_{x}
$$

other physically important cases are the cubic one $f(s)=s^{3}$, and the quadratic-cubic nonlinearity, namely $f(s)=s^{2}-\mu s^{3}, \mu \in \mathbb{R}$. In the former case, the equation (11) is often refered as the (focusing) modified $\mathrm{KdV}$ equation $(\mathrm{mKdV})$, and in the latter, it is known as the Gardner equation.

Concerning the KdV equation, it arises in Physics as a model of propagation of dispersive long waves, as was pointed out by J. S. Russel in 1834 [27. The exact formulation of the KdV equation comes from Korteweg and de Vries (1895) [15. This equation was re-discovered in a numerical work by N. Zabusky and M. Kruskal in 1965 [34.

After this work, a great amount of literature has emerged, physical, numerical and mathematical, for the study of this equation, see for example [5, 14, 30, 18, 9, 28, 27]. Although under different points of view, among the main topics treated are the following: existence of explicit solutions and their stability, local and global well posedness, long time behavior properties and, of course, related generalized models, hierarchies and their properties.

This continuous, focused research on the KdV equation can be in part explained by some striking algebraic properties. One of the first properties is the existence of localized, rapidly decaying, stable and smooth solutions called solitons. Given three real numbers $t_{0}, x_{0}$ and $c>0$, solitons are solutions of (2) of the form

$$
u(t, x):=Q_{c}\left(x-x_{0}-c\left(t-t_{0}\right)\right), \quad Q_{c}(s):=c Q\left(c^{1 / 2} s\right),
$$

and where $Q$ satisfies the second order nonlinear differential equation

$$
Q^{\prime \prime}-Q+Q^{2}=0, \quad Q(x)=\frac{3}{2 \cosh ^{2}\left(\frac{1}{2} x\right)} .
$$

Date: February, 2009.

2000 Mathematics Subject Classification: Primary 35Q51, 35Q53; Secondary 37K10, 37K40.

Keywords and phrases: KdV equation, Integrability theory, Collision of solitons.

This research was supported in part by a CONICYT-Chile and an Allocation de Recherche grants. 
The 3-parameter family of solitons (3) contains three important symmetries of the equation, namely scaling and translation in space and time invariances. From the Noëther theorem, these two last symmetries are related to conserved quantities, invariant under the KdV flow, usually called Mass and Energy, represented below in (7)-(8) (in a general form). Moreover, due to the mass and energy conservation, the Sobolev space $H^{1}(\mathbb{R})$ appears as an ideal space to study long time properties of $\mathrm{KdV}$.

Even more striking is the fact that $\mathrm{KdV}, \mathrm{mKdV}$ and the Gardner equation, being infinite dimensional dynamical systems, possess an infinity number of conserved quantities, a consequence of the so-called complete integrability property. This one is closely related to the existence of a Lax pair for these equations (see Lax, [17]). Another important property is the following well known fact: given any Schwartz initial data, the corresponding solution to the Cauchy problem for (2) exists globally in time and decouples, as $t \rightarrow+\infty$, into a radiation part going leftward plus a nonlinear multisoliton component going to the right, see [31.

The dynamical problem of 2 -soliton collision is a classical problem in nonlinear wave propagation (see 24] for a review and references therein). By 2-soliton collision we mean the following problem: given two solitons, solutions of (11), largely separated at some early time and having different velocities, we expect that they have to collide at some finite time. The resulting solution after the collision is precisely the object of study. In particular, one considers if any change in size, position, or shape, even destruction of the solitons, after some large time, may be present.

Let us review some relevant works in this direction. First, the works of Fermi, Pasta and Ulam [7] and Zabusky and Kruskal 34 exhibited numerical results showing a remarkable phenomena related to solitons collision. More precisely, they put in evidence the elastic character of the collision between two solitons. By elastic we mean that collision keeps the solitons unchanged and does not produce any residual term of positive mass for large times. The unique consequence of the collision is a shift translation on each soliton, depending on their sizes. Next, the work of Lax [17] developed a mathematical framework to study these problems. After this, the inverse scattering method (we refer e.g. to [1] and 27] for a review) provided explicit formulas for $N$-soliton solutions (Hirota [10]). Indeed, let $c_{1}>c_{2}>0$ and $\delta_{1}, \delta_{2} \in \mathbb{R}$ be arbitrary given numbers. There exists an explicit solution $U=U_{c_{1}, c_{2}}(t, x)$ of (2) which satisfies

$$
\left\|U(t, \cdot)-\sum_{j=1}^{2} Q_{c_{j}}\left(\cdot-c_{j} t-\delta_{j}\right)\right\|_{H^{1}(\mathbb{R})} \underset{t \rightarrow-\infty}{\longrightarrow} 0, \quad\left\|U(t, \cdot)-\sum_{j=1}^{2} Q_{c_{j}}\left(\cdot-c_{j} t-\delta_{j}^{\prime}\right)\right\|_{H^{1}(\mathbb{R})} \underset{t \rightarrow+\infty}{\longrightarrow} 0,
$$

for some $\delta_{j}^{\prime}$ such that the shifts $\Delta_{j}=\delta_{j}^{\prime}-\delta_{j}$ depend only on $c_{1}, c_{2}$. This solution, called 2-soliton, represents the pure collision of two solitons, with no residual terms before and after the collision. In other words, the collision is elastic.

These properties are also valid for the cubic mKdV, (see [1, p. 390) and for the Gardner equation (see [8, 32] and references there in). In particular, complete integrability and elastic collisions are still present. Let us recall that for the Gardner equation

$$
u_{t}+\left(u_{x x}+u^{2}-\mu u^{3}\right)_{x}=0,
$$

given $\mu \in \mathbb{R}$, soliton solutions exist for all $c>0$ in the case $\mu<0$, and provided $c<\frac{2}{9 \mu}$ if $\mu>0$. These solutions are explicit and given by $u(t, x)=Q_{\mu, c}(x-c t)$, where $Q_{\mu, c}$ is the Schwartz function 32 .

$$
Q_{\mu, c}(x):=\frac{3 c}{1+\rho \cosh (\sqrt{c} x)} ; \quad \rho:=\left(1-\frac{9}{2} \mu c\right)^{1 / 2} .
$$

In particular, no soliton-solution exists provided $\mu>0$, and $c>0$ large enough, where the character of the equation becomes defocusing.

We point out that these techniques are known to be too rigid to be applied to more general models, and have no equivalent for the case of the gKdV equation (11) with a general nonlinearity. The first purpose of this paper is to confirm this belief under reasonable hypothesis on the nonlinearity: the collision of two solitons is not elastic in general, except by $\mathrm{KdV}, \mathrm{mKdV}$ and the Gardner equations. Before establishing our main result we explain the framework where the problem must be posed. 
The complete integrability property has been studied in many other differential equations, as NLS, KPI, Benjamin-Ono, etc.; see for example [1]. In particular, when complete integrability is lost, very little is known. We mention the recent works of Perelman [29], Holmer, Marzuola and Zworski [11, 12, 13] and Abou Salem, Fröhlich and Sigal [3] on the problem of 2-soliton collision for the nonlinear Schrödinger equation (NLS) under the action of a potential and considering higher velocities.

1.1. Setting and hypothesis. Let us come back to the general equation (1). Assume that the nonlinearity $f \in C^{3}(\mathbb{R})$. The Cauchy problem for equation (1) (namely, adding the initial condition $\left.u(t=0)=u_{0}\right)$ is locally well-posed for $u_{0} \in H^{1}(\mathbb{R})$ (see Kenig, Ponce and Vega [16]).

For $H^{1}(\mathbb{R})$ solutions, in the general case, unlike the integrable cases, only the following two quantities are conserved by the flow:

$$
M(t):=\int_{\mathbb{R}} u^{2}(t, x) d x=\int_{\mathbb{R}} u_{0}^{2}(x) d x=M(0), \quad \text { (Mass), }
$$

and

$$
\begin{aligned}
E(t):=\frac{1}{2} & \int_{\mathbb{R}} u_{x}^{2}(t, x) d x-\int_{\mathbb{R}} F(u(t, x)) d x \\
& =\frac{1}{2} \int_{\mathbb{R}}\left(u_{0}\right)_{x}^{2}(x) d x-\int_{\mathbb{R}} F\left(u_{0}(x)\right) d x=E(0), \quad(\text { Energy })
\end{aligned}
$$

where we have denoted

$$
F(s):=\int_{0}^{s} f(\sigma) d \sigma .
$$

In the case of a pure power $f(s)=s^{m}, m<5$, any $H^{1}(\mathbb{R})$ solution is global in time thanks to the conservation of energy (8). For $m=5$, solitons are shown to be unstable and the Cauchy problem for the corresponding gKdV equation has finite-time blow-up solutions, and see [21] and references there in. It is believed that for $m>5$ the situation is the same. The origin grosso modo of this instability comes from the lack of control for the injection $H^{1}(\mathbb{R}) \rightarrow L^{p}(\mathbb{R})$ for $p \geq 5$. Indeed, from the Galiardo-Nirenberg inequality

$$
\int_{\mathbb{R}}|v|^{p+1} \leq C(p)\left(\int_{\mathbb{R}} v^{2}\right)^{\frac{p+3}{4}}\left(\int_{\mathbb{R}} v_{x}^{2}\right)^{\frac{p-1}{4}},
$$

valid for any $v \in H^{1}(\mathbb{R})$, one can see that the energy (8) cannot be controlled by the usual $H^{1}$-norm. Consequently, in this work, we will discard high-order nonlinearities at leading order. Indeed, we will consider nonlinearities $f$ of the form

$$
f \in C^{m+2}(\mathbb{R}), \quad f(u):=u^{m}+f_{1}(u), \quad m=2,3,4, \quad \text { with } \quad \lim _{s \rightarrow 0} \frac{\left|f_{1}(s)\right|}{|s|^{m}}=0 .
$$

Moreover, using stability properties of the solitons, we will have only global in time solutions, namely $u(t) \in H^{1}(\mathbb{R})$ for all time $t \in \mathbb{R}$.

The positive sign leading in front of $f$ (see (10)) allows the existence of solitons for (21) of the form

$$
u(t, x):=Q_{c}\left(x-x_{0}-c t\right),
$$

with $c>0$ small enough and $x_{0} \in \mathbb{R}$, where the function $Q_{c}$ satisfies the elliptic equation

$$
Q_{c}^{\prime \prime}+f\left(Q_{c}\right)=c Q_{c}, \quad Q_{c} \in H^{1}(\mathbb{R}) .
$$

From Berestycki and Lions [4] and (1), it follows that there exists $c_{*}(f)>0$ (possibly $\left.+\infty\right)$ defined by

$$
c_{*}(f):=\sup \left\{c>0 \text { such that for all } c^{\prime} \in(0, c) \text {, exists } Q_{c^{\prime}} \text { positive solution of (11) }\right\} .
$$

For all $c>0$, if a solution $Q_{c}>0$ of (11) exists then it can be chosen even on $\mathbb{R}$ and exponentially decreasing on $\mathbb{R}^{+}$(and similarly if $Q_{c}<0$ ). Moreover, in [22], the authors have showed that $0<c<c_{*}(f)$ is a sufficient condition for asymptotic stability in the energy space $H^{1}$ around the soliton $Q_{c}$, see also Proposition 3.3 for details. 
Finally, in this paper, we consider only nonlinear stable solitons in the sense of Weinstein 33, i.e. such that

$$
\left.\frac{d}{d c^{\prime}} \int Q_{c^{\prime}}^{2}(x) d x\right|_{c^{\prime}=c}>0 .
$$

Note that since $m=2,3,4$ in (10), this condition is automatically satisfied for $c>0$ small enough (in the pure power case $f(s)=s^{m}$, this condition is satisfied for any $c>0$ provided $m<5$, see [33).

1.2. Previous analytic results on 2-soliton collision in non-integrable cases. As pointed out in 24, the problem of describing the collision of two traveling waves or solitons is a general problem for nonlinear PDEs, which is almost completely open, except in the integrable cases described above. On the other hand, these problems have been studied since the 60 's from both experimental and numerical points of view.

We deal with these questions for (11) with a general nonlinearity $f(u)$ in a particular setting: we consider two positive solitons $Q_{c_{1}}, Q_{c_{2}}, 0<c_{2}<c_{1}<c_{*}(f)$, and we assume $c_{2}$ small compared with $c_{1}$.

Under these assumptions, Martel and Merle 24] considered the collision problem for (28) in the quartic case, $f(s)=s^{4}$, with one soliton small with respect to the other. They showed that the collision is almost elastic, but inelastic, by showing the nonexistence of pure 2-soliton solution.

Theorem 1.1 (Non-existence of a pure 2-soliton solution, quartic case 24]). Let $f(s):=s^{4}$ and $0<c:=\frac{c_{2}}{c_{1}}<1$. There exists a constant $c_{0}>0$ such that if $c<c_{0}$ then the following holds. Let $u(t) \in H^{1}(\mathbb{R})$ be the unique solution of (1) such that

$$
\lim _{t \rightarrow-\infty}\left\|u(t)-Q_{c_{1}}\left(\cdot-c_{1} t\right)-Q_{c_{2}}\left(\cdot-c_{2} t\right)\right\|_{H^{1}(\mathbb{R})}=0 .
$$

Then there exist $x_{1}^{+}, x_{2}^{+}, c_{1}^{+}>c_{2}^{+}>0$ and constants $T_{0}, K>0$ large enough such that

$$
w^{+}(t, \cdot):=u(t, \cdot)-Q_{c_{1}^{+}}\left(\cdot-x_{1}^{+}-c_{1}^{+} t\right)-Q_{c_{2}^{+}}\left(\cdot-x_{2}^{+}-c_{2}^{+} t\right)
$$

satisfies

(1) Support on the left of solitons.

$$
\lim _{t \rightarrow+\infty}\left\|w^{+}(t)\right\|_{H^{1}\left(x>\frac{1}{10} c_{2} t\right)}=0 .
$$

(2) Parameters perturbation. The limit scaling parameters $c_{1}^{+}$and $c_{2}^{+}$satisfy

$$
\frac{1}{K} c^{\frac{17}{6}} \leq \frac{c_{1}^{+}}{c_{1}}-1 \leq K c^{\frac{11}{6}}, \quad \text { and } \quad \frac{1}{K} c^{\frac{8}{3}} \leq 1-\frac{c_{2}^{+}}{c} \leq K c^{\frac{1}{3}} .
$$

In particular, $c_{1}^{+}>c_{1}$ and $c_{2}^{+}<c_{2}$.

(3) Non zero residual term. For every $t \geq T_{0}$, the adapted $H^{1}$-norm of $w^{+}(t)$ satisfies

$$
\frac{1}{K} c_{1}^{\frac{7}{12}} c^{\frac{17}{12}} \leq\left\|w_{x}^{+}(t)\right\|_{L^{2}(\mathbb{R})}+\sqrt{c_{1} c}\left\|w^{+}(t)\right\|_{L^{2}(\mathbb{R})} \leq K c_{1}^{\frac{7}{12}} c^{\frac{11}{12}} .
$$

Remark 1.1. The existence and uniqueness of the solution of (1) satisfying (13) was proved in [19].

Remark 1.2. Note that $\left\|Q_{c_{2}}\right\|_{H^{1}(\mathbb{R})} \sim c^{\frac{1}{12}} \gg K c^{\frac{5}{12}} \geq\left\|w^{+}(t)\right\|_{L^{2}(\mathbb{R})}$ for $c$ small. In other words the defect $w^{+}$is really small compared with $Q_{c_{2}}$.

The next question arising from this result is to generalize these results to (11) under assumption (10). In this case, Martel and Merle [25] proved that the collision is still stable, giving upper bounds on the residual terms appearing after the collision. In particular, their result extends the positive part of Theorem 1.1 .

Theorem 1.2 (Behavior after collision of a pure 2-soliton solution, 25]).

Let $f$ satisfying (10). Let $0<c_{2}<c_{1}<c_{*}(f)$ be such that the positive solution $Q_{c_{1}}$ of (11) satisfies (12). Then there exists $c_{0}=c_{0}\left(c_{1}\right) \in\left(0, c_{1}\right)$ such that if $c_{2}<c_{0}\left(c_{1}\right)$ then the following holds. Let $u(t)$ be the solution of (21) satisfying

$$
\lim _{t \rightarrow-\infty}\left\|u(t)-Q_{c_{1}}\left(\cdot-c_{1} t\right)-Q_{c_{2}}\left(\cdot-c_{2} t\right)\right\|_{H^{1}(\mathbb{R})}=0 .
$$


Then, there exist $\rho_{1}(t), \rho_{2}(t), c_{1}^{+}>c_{2}^{+}>0$ and $K>0$ such that

$$
\begin{gathered}
w^{+}(t, x):=u(t, x)-Q_{c_{1}^{+}}\left(x-\rho_{1}(t)\right)-Q_{c_{2}^{+}}\left(x-\rho_{2}(t)\right) \\
\text { satisfies } \sup _{t \in \mathbb{R}}\left\|w^{+}(t)\right\|_{H^{1}(\mathbb{R})} \leq K c_{2}^{\frac{1}{m-1}} \text { and for } q=q_{m}:=\frac{2}{m-1}+\frac{1}{4}, \\
\lim _{t \rightarrow+\infty}\left\|w^{+}(t)\right\|_{H^{1}\left(x>\frac{1}{10} c_{2} t\right)}=0, \quad \limsup _{t \rightarrow+\infty}\left\|w^{+}(t)\right\|_{H^{1}(\mathbb{R})} \leq K c_{2}^{q-\frac{1}{2}-\frac{1}{100}}, \\
\lim _{t \rightarrow+\infty}\left|\rho_{1}^{\prime}(t)-c_{1}^{+}\right|+\left|\rho_{2}^{\prime}(t)-c_{2}^{+}\right|=0 .
\end{gathered}
$$

Moreover, $\lim _{t \rightarrow+\infty} E\left(w^{+}(t)\right)=: E^{+}$and $\lim _{t \rightarrow+\infty} \int_{\mathbb{R}}\left(w^{+}\right)^{2}(t)=: M^{+}$exist and the following bounds hold

$$
\frac{1}{2} \limsup _{t \rightarrow+\infty} \int_{\mathbb{R}}\left(\left(w_{x}^{+}\right)^{2}+c_{2}\left(w^{+}\right)^{2}\right)(t) \leq 2 E^{+}+c_{2} M^{+} \leq \liminf _{t \rightarrow+\infty} \int_{\mathbb{R}}\left(\left(w_{x}^{+}\right)^{2}+2 c_{2}\left(w^{+}\right)^{2}\right)(t) .
$$

Finally, the limit parameters $c_{1}^{+}$and $c_{2}^{+}$satisfy the following bounds

$$
\frac{1}{K}\left(2 E^{+}+c_{2} M^{+}\right) \leq \frac{c_{1}^{+}}{c_{1}}-1 \leq K\left(2 E^{+}+c_{2} M^{+}\right),
$$

and

$$
\frac{1}{K} c_{2}^{q-\frac{3}{4}}\left(2 E^{+}+c_{1} M^{+}\right) \leq 1-\frac{c_{2}^{+}}{c_{2}} \leq K c_{2}^{q-\frac{3}{4}}\left(2 E^{+}+c_{1} M^{+}\right) .
$$

Remark 1.3. In Theorem 1.2, if $c_{1}^{+}=c_{1}$ and $c_{2}^{+}=c_{2}$ (or equivalently $E^{+}=M^{+}=0$ ), then the solution $u(t)$ is a pure 2-soliton solution and the collision is elastic.

In 25, the question of whether the collision is elastic or inelastic in the general case -and thus the nonexistence of pure 2-soliton solutions- was left open, see [25], Remark 1. More precisely, the authors conjectured a classification result concerning the nonlinearities $f(s)$ allowing small stable solitons. This affirmation asserts that under reasonable stability properties, the unique nonlinearities for which any 2-soliton collision is pure are the integrable cases, $f(s)=s^{2}, f(s)=s^{3}$ and a linear combination of both nonlinearities. Theorem 1.1 from [24] was the first step in this direction. By extending some techniques from [24, 25] and developping new computations, we are able to provide a satisfactory answer to this open question.

1.3. Main results. Consider the framework introduced in Theorem 1.2 . In addition to this result, we have the following

Theorem 1.3 (Non-existence of pure 2-soliton solution, general case). Let $f$ be as in (10), with $m=2$ or 3 , and

$$
f \in C^{p+1}(\mathbb{R}), \quad f^{(p)}(0) \neq 0 \quad \text { for some } \quad p \geq 4 .
$$

For $0<c_{2} \ll c_{1} \ll 1$ equation (1) has no pure 2-soliton solution of sizes $c_{1}, c_{2}$. In particular Theorem 1.2 holds with $c_{1}^{+}>c_{1}$ and $c_{2}^{+}<c_{2}$.

Remark 1.4. The nonzero condition $f^{(p)}(0) \neq 0$ for some $p \geq 4$ rules out the integrable cases $f(s)=s^{m}, m=2$ or 3 and the Gardner nonlinearity $f(s)=s^{2}-\mu s^{3}$.

Remark 1.5. We do not treat the degenerate cases $f(s)=s^{m}+f_{1}(s)$, for $f_{1}(s) \neq 0$ but $f_{1}^{(p)}(0)=0$ for all $p \geq 4$. These cases seem to be not physically relevant.

Remark 1.6. The result of Theorem 1.3 in the quartic case $m=4$ follows directly from the proof of Theorem 1.1] in 24] together with the techniques used in the present paper. This remark and Theorem 1.3 allow to classify the nonlinearities for which 2 -soliton collision is elastic. In particular, with the restriction mentioned in Remark 1.5. we obtain that pure 2-soliton solutions are present for any pair of solitons with different velocities if and only if $f$ corresponds to the integrable cases, $f(s)=s^{2}, s^{3}$ and linear combinations. We recall that for $m \geq 5$ in (10), solitons have been shown to be unstable (see [6]). It is believed that collision may produce blow-up solutions in finite time. 
Theorem 1.3 is a consequence of the following reduction of the problem. Let $p$ be the smallest integer greater or equal than 4 satisfying (20). Let $c_{1}>0$ small. Consider the transformation

$$
\tilde{u}(t, x):=c_{1}^{-\frac{1}{m-1}} u\left(c_{1}^{-\frac{3}{2}} t, c_{1}^{-\frac{1}{2}} x\right),
$$

which maps $Q_{c_{1}}\left(x-c_{1} t\right)$ to $Q(x-t)$ and $Q_{c_{2}}\left(x-c_{2} t\right)$ to $Q_{c}(x-c t)$, with $c:=\frac{c_{2}}{c_{1}}$. If $u=u(t, x)$ is solution of (1) then $\tilde{u}$ is solution of the equation

$$
\left\{\begin{array}{l}
\tilde{u}_{t}+\left(\tilde{u}_{x x}+\tilde{f}(\tilde{u})\right)_{x}=0, \\
\text { with } \tilde{f}(\tilde{u}):=\tilde{u}^{m}+\tilde{f}_{1}(\tilde{u}), \quad \tilde{f}_{1}(\tilde{u}):=c_{1}^{-\frac{m}{m-1}} f_{1}\left(c_{1}^{\frac{1}{m-1}} \tilde{u}\right) .
\end{array}\right.
$$

Note that $\tilde{f}_{1}$ satisfies (10). Then, for the case $m=3, \tilde{f}_{1}$ can be expanded as

$$
\begin{aligned}
\tilde{f}_{1}(\tilde{u}) & =c_{1}^{-\frac{3}{2}}\left[\frac{1}{p !} f_{1}^{(p)}(0)\left(c_{1}^{\frac{1}{2}} \tilde{u}\right)^{p}+O\left(\left(c_{1}^{\frac{1}{2}} \tilde{u}\right)^{p+1}\right)\right] \\
& =: \quad \varepsilon \tilde{u}^{p}+|\varepsilon|^{1+\frac{1}{p-3}} \hat{f}_{1}(\tilde{u}),
\end{aligned}
$$

where $\varepsilon:=\frac{1}{p !} f_{1}^{(p)}(0) c_{1}^{\frac{p-3}{2}}$ is small and $\hat{f}_{1}$ satisfies the decay relation $\lim _{s \rightarrow 0}|s|^{-p} \hat{f}_{1}(s)=0$.

For the quadratic case, we need more care because of the Gardner nonlinearity. We have

$$
\begin{aligned}
\tilde{f}_{1}(\tilde{u}) & =c_{1}^{-2}\left[\frac{1}{6} f_{1}^{(3)}(0)\left(c_{1} \tilde{u}\right)^{3}+\frac{1}{p !} f_{1}^{(p)}(0)\left(c_{1} \tilde{u}\right)^{p}+O\left(\left(c_{1} \tilde{u}\right)^{p+1}\right)\right] \\
& =\frac{1}{6} f_{1}^{(3)}(0) c_{1} \tilde{u}^{3}+\frac{1}{p !} f_{1}^{(p)}(0) c_{1}^{p-2} \tilde{u}^{p}+O\left(c_{1}^{p-1} \tilde{u}^{p+1}\right) \\
& =: \mu(\varepsilon) \tilde{u}^{3}+\varepsilon \tilde{u}^{p}+|\varepsilon|^{1+\frac{1}{p-2}} \hat{f}_{1}(\tilde{u}),
\end{aligned}
$$

where $\varepsilon:=\frac{1}{p !} f_{1}^{(p)}(0) c_{1}^{p-2} \neq 0$ by hypothesis, and $\mu(\varepsilon):=\frac{1}{6} f_{1}^{(3)}(0) c_{1}=\hat{\mu} \varepsilon^{\frac{1}{p-2}}, \hat{\mu} \in \mathbb{R}$. Here both $\varepsilon$ and $\mu$ are small (depending on $c_{1}$ ) and $\hat{f}_{1}$ satisfies the decay relation $\lim _{s \rightarrow 0}|s|^{-p} \hat{f}_{1}(s)=0$. Note that in this framework, the quadratic case can be seen as a particular case of the Gardner nonlinearity, for which $\hat{\mu}=0$.

Finally, we drop the tilde on $\tilde{u}$ and $\tilde{f}$ and the hat on $\hat{f}_{1}$. We are now reduced to the $\varepsilon$-dependent equation

$$
u_{t}+\left(u_{x x}+f(u)\right)_{x}=0
$$

where $\mu(\varepsilon)=\hat{\mu} \varepsilon^{\frac{1}{p-2}}, \hat{\mu} \in \mathbb{R}$,

$$
f=f_{\varepsilon} \in C^{p+1}(\mathbb{R}), f(u)=\left\{\begin{array}{l}
u^{2}+\mu(\varepsilon) u^{3}+\varepsilon u^{p}+\varepsilon^{1+\frac{1}{p-2}} f_{1}(u), m=2, \\
u^{3}+\varepsilon u^{p}+\varepsilon^{1+\frac{1}{p-3}} f_{1}(u), m=3,
\end{array} \lim _{s \rightarrow 0} \frac{f_{1}(s)}{|s|^{p}}=0,\right.
$$

and $\varepsilon$ is small, and $p \geq 4$. For notational commodity we will skip the $\varepsilon$-dependence on the functions considered along this work, except in some computations performed in Appendix B, Lastly, note that for $\varepsilon$ small $Q$ and $Q_{c}$ satisfy (12), see also Remark 3.2 .

In this framework, we now claim the main result of this paper:

Theorem 1.4 (Non-existence of pure 2-soliton solution, general case). Suppose $m=2,3$ and $f$ satisfying (22) for $p \geq 4$. There exists a constant $\varepsilon_{0}>0$ such that if

$$
0<|\varepsilon|<\varepsilon_{0}, \quad \text { and } \quad 0<c \leq|\varepsilon|^{m-1+\frac{1}{25}},
$$

then the following holds. Let $u(t)$ be solution of (21) satisfying

$$
\lim _{t \rightarrow-\infty}\left\|u(t)-Q(\cdot-t)-Q_{c}(\cdot-c t)\right\|_{H^{1}(\mathbb{R})}=0
$$

there exist $K, T_{0}>0$ such that

(1) Non zero residual term. There exist $\rho_{1}(t), \rho_{2}(t), c_{1}^{+}>c_{2}^{+}>0$ such that

$$
w^{+}(t):=u(t, x)-Q_{c_{1}^{+}}\left(x-\rho_{1}(t)\right)-Q_{c_{2}^{+}}\left(x-\rho_{2}(t)\right)
$$

satisfies, for every $t \geq T_{0}$,

$$
\lim _{t \rightarrow+\infty}\left\|w^{+}(t)\right\|_{H^{1}\left(x>\frac{1}{10} c t\right)}=0,
$$




$$
\begin{aligned}
& \text { and for } q= \frac{2}{m-1}+\frac{1}{4}, \\
& \qquad \frac{1}{K}|\varepsilon| c^{q+\frac{1}{2}} \leq\left\|w_{x}^{+}(t)\right\|_{L^{2}(\mathbb{R})}+\sqrt{c}\left\|w^{+}(t)\right\|_{L^{2}(\mathbb{R})} \leq K|\varepsilon| c^{q} .
\end{aligned}
$$

(2) The asymptotic scaling parameters $c_{1}^{+}$and $c_{2}^{+}$satisfy

$$
\begin{aligned}
& \frac{1}{K} \varepsilon^{2} c^{2 q+1} \leq c_{1}^{+}-1 \leq K \varepsilon^{2} c^{2 q}, \text { and } \\
& \frac{1}{K} \varepsilon^{2} c^{3 q+\frac{1}{4}} \leq 1-\frac{c_{2}^{+}}{c} \leq K \varepsilon^{2} c^{3 q-\frac{7}{4}} .
\end{aligned}
$$

Before sketching the proof of this Theorem, some remarks are in order.

Remark 1.7 (Comments on the assumptions). In the present paper, as in [24, 25, we study the collision of two solitons $Q_{c_{1}}$ and $Q_{c_{2}}$. The assumption $c_{2}$ small allows to linearize in $c_{2}$, and then to reduce the non existence of a pure 2 -soliton solution to the computation of a coefficient depending only on $c_{1}$. For general $f$, as in (10), and general $c_{1}>0$, it is an open question to compute this coefficient, see Remark 2.7 (for $p=4$, a special algebraic structure allowed to compute this coefficient, see [24]).

According to this, we compute the asymptotics of this coefficient as $c_{1}$ is small (or equivalent, $\varepsilon$ is small), see Appendix B. This is the only place where $\varepsilon$ small is needed. This asymptotic allows us to conclude under the additional restriction $0<c<|\varepsilon|^{m-1+\frac{1}{25}}$ (see Proposition 2.11] and estimate (105)). The exponent $\frac{1}{25}$ has no special meaning, and can be taken as small as we want, as long as $c$ is taken even smaller.

Two open questions then arise:

(1) Can we relax in (23) the second condition on $c$ ?

(2) For general $f$, do there exist special values of $c_{1}$ for which the coefficient is zero? The residue from the collision would then be of smaller order in $c_{2}$.

Remark 1.8. For $m=2$, the smoothness condition (22) allows nonlinearities of type $f(s)=$ $s^{2}+\nu \varepsilon^{p-2} s^{p}$, with $p \geq 4$ (possibly non integer). For $m=3$, the same conclusion follows for nonlinearities of the type $f(s)=s^{3}+\varepsilon s^{p}, p=4$ and $p \geq 5$ (possibly non integer). See also Appendix $\mathrm{A}$ and final remarks in Appendix B.

Remark 1.9. Although this theorem asserts that collision is indeed inelastic, near-elastic, the appearance of smaller solitons on the left of the solitons is not discarded by our proof, and (25). However, we believe that, at least under the condition of Theorem 1.4 there are no such small solitons.

1.4. Sketch of the proof. Our proof will follow closely the approach described by Martel, Merle and Mizumachi [24, 25, 26. The argument is as follows: we consider the solution (unique, see [19] $u(t)$ of (21) satisfying (14) at time $t \sim-\infty$. Then, we separate the analysis among three different time intervals: $t \ll-c^{-\frac{1}{2}},|t| \leq c^{-\frac{1}{2}}$ and $c^{-\frac{1}{2}} \ll t$. On each interval the solution possesses a specific behavior which we briefly describe:

(1) $\left(t \ll-c^{-\frac{1}{2}}\right)$. In this interval of time we prove that $u(t)$ remains close to a 2 -soliton solution with no changes on scaling and shift parameters. This result is possible for negative long enough times, such that both solitons are still far from each other, and is a consequence of [19].

(2) $\left(|t| \leq c^{-\frac{1}{2}}\right)$. This is the interval where solitons collision leads the dynamic of $u(t)$. The novelty in the method is the construction of an approximate solution of (21) with high order of accuracy such that $(a)$ at time $t \sim-c^{-\frac{1}{2}}$ this solution is close to a 2-soliton solution and therefore to $u(t),(b)$ it describes the 2 -soliton collision in this interval, $(c)$ at time $t \sim c^{-\frac{1}{2}}$, when solitons are sufficiently separated, it possesses an extra, nonzero, residual term product of the collision, and characterized by a number $d(\varepsilon) \neq 0$ (cf. (56)$(57))$, and $(d)$ it is possible to extend the solution $u(t)$ to the whole interval $\left[-c^{\frac{1}{2}}, c^{\frac{1}{2}}\right]$ being still close to our approximate solution, uniformly on time, modulo modulation on 
a translation parameter. This property confirms that our Ansatz is indeed the correct approximate solution describing the collision.

(3) $\left(t \gg c^{-\frac{1}{2}}\right)$ Here some stability properties (see Proposition 3.3) will be used to establish the convergence of the solution $u(t)$ to a 2 -soliton solution with modified parameters. Moreover, by using a monotony argument, it will be possible to show that the residue appearing after the collision at time $t \sim c^{-\frac{1}{2}}$ is still present at infinity. This gives the conclusion of the Theorem.

The plan of this paper is as follows. In Section 2 we construct the aforementioned approximate solution and compute the error term produced in terms of a set of linear problems. Then we solve such linear systems and finally we give the first basic estimates concerning this solution. We finally prove that it is indeed close to a 2 -soliton solution. In section 3 we construct an actual solution $u$ close to the approximate solution for small times, and state some stability results to study the long time behavior of the solution $u$. Finally, in section 4 , we prove Theorem using above results.

\section{Construction of an approximate 2-SOliton solution}

The objective of this section is to construct an approximate solution of the gKdV equation (21),

which will precisely describe the collision of two solitons. Hereafter, we assume the hypothesis of Theorem 1.4. We suppose both solitons are positive (the negative case, for $m=3$, can be treated in the same way).

Secondly, note that $Q$ and $Q_{c}$ have velocity (and size) 1 and $c$ respectively; so that working with $u(t, x+t)$ instead of $u(t, x)$ we can assume that the great soliton $Q$ is fixed at $x=0$ and the small soliton has velocity $c-1<0$. Of course, $v(t, x):=u(t, x+t)$ satisfies now the translated equation

Finally, denote

$$
v_{t}+\left(v_{x x}-v+f(v)\right)_{x}=0 \quad \text { on } \mathbb{R}_{t} \times \mathbb{R}_{x} .
$$

$$
T_{c}:=c^{-\frac{1}{2}-\frac{1}{100}}>0 .
$$

This quantity can be understood as the time of interaction between the two solitons. The exponent $\frac{1}{100}$ can be replaced by any small positive number without relevant modifications.

The following result deals with the problem of describing the collision in the interval of time $\left[-T_{c}, T_{c}\right]$ :

Proposition 2.1 (Construction of an approximate solution of the gKdV equation). Let $m=2,3$ and $f$ as in (22). There exist constants $c_{0}=c_{0}(f)>0$ and $K_{0}=K_{0}(f)$ such that for all $0<c<c_{0}$ there exists a function $\tilde{u}=\tilde{u}_{1, c}(t, x)$ such that the following hold:

(1) Approximate solution on $\left[-T_{c}, T_{c}\right]$. For all $t \in\left[-T_{c}, T_{c}\right]$,

$$
\left\|\tilde{u}_{t}+\left(\tilde{u}_{x x}-\tilde{u}+f(\tilde{u})\right)_{x}\right\|_{H^{2}(\mathbb{R})} \leq K_{0} c^{\frac{3}{m-1}+\frac{3}{4}} .
$$

(2) Closeness to the sum of two solitons: For all time $t \in\left[-T_{c}, T_{c}\right]$, the function $\tilde{u}$ belongs to $H^{1}(\mathbb{R})$ and satisfies

$$
\left\|\tilde{u}(t)-Q(x-\alpha)-Q_{c}(x+(1-c) t)\right\|_{H^{1}(\mathbb{R})} \leq K_{0} c^{\frac{1}{m-1}},
$$

where $\alpha=\alpha(t, x)$ is a smooth bounded function, to be defined below, see (31).

Remark 2.1. The proof of this proposition requires several steps, starting in Subsection 2.1 to finally ending in Subsection 2.3. Proposition 2.10. However, the proof is intuitively clear to describe: our approximate solution will consists of a linear combination of a nonlinear basis well behaved under the gKdV flow, together a variable decomposition resembling the classical separation of variables from second order linear PDEs. This description was first introduced by Martel and Merle 24, 25].

First of all we explain how the approximate solution is composed. We follow [24]. 
2.1. Decomposition of the approximate solution. We look for $\tilde{u}(t, x)$, the approximate solution for (28), carring out a specific structure. We first introduce a set of indices, depending on the cases we deal with. Let

$$
\Sigma_{2}:=\{(k, l)=(1,0),(1,1),(2,0),(2,1),(1,2),(3,0)\},
$$

for the quadratic case $(m=2)$, and

$$
\Sigma_{3}:=\{(k, l)=(1,0),(1,1),(2,0),(2,1),(3,0),(4,0)\},
$$

for the cubic one $(m=3)$.

We recall now an order relation for indices $(k, l),\left(k^{\prime}, l^{\prime}\right) \in \Sigma_{m}$ introduced in 24. We say that

$$
\left(k^{\prime}, l^{\prime}\right)<(k, l) \text { if and only if }\left\{\begin{array}{l}
k^{\prime}<k \text { and } l^{\prime} \leq l, \text { or } \\
k^{\prime} \leq k \text { and } l^{\prime}<l .
\end{array}\right.
$$

We set two variables denoting the position of each soliton. For the small soliton, let

$$
y_{c}:=x+(1-c) t \quad \text { and } \quad R_{c}(t, x):=Q_{c}\left(y_{c}\right),
$$

and for the great soliton,

$$
y:=x-\alpha\left(y_{c}\right) \quad \text { and } \quad R(t, x):=Q(y)
$$

where for $\left(a_{k, l}\right)_{(k, l) \in \Sigma_{m}}$,

$$
\alpha(s):=\int_{0}^{s} \beta\left(s^{\prime}\right) d s^{\prime}, \quad \beta(s):=\sum_{(k, l) \in \Sigma_{m}} a_{k, l} c^{l} Q_{c}^{k}(s) .
$$

The correction term $\alpha$ is intended to describe the shift on the position of the great soliton. Note that $\alpha$ might be nonzero even in the integrable case, see (4). Moreover, in the quartic case $m=4, \varepsilon=0$, one has $|\alpha| \rightarrow+\infty$ as $c \rightarrow 0$, see [22]. Along this work $\alpha$ will be a bounded function, uniformly on $c$.

The form of $\tilde{u}(t, x)$ is, as it should be expected, the sum of the two soliton plus a correction term:

$$
\begin{gathered}
\tilde{u}(t, x):=Q(y)+Q_{c}\left(y_{c}\right)+W(t, x), \\
W(t, x):=\sum_{(k, l) \in \Sigma_{m}} c^{l}\left(Q_{c}^{k}\left(y_{c}\right) A_{k, l}(y)+\left(Q_{c}^{k}\right)^{\prime}\left(y_{c}\right) B_{k, l}(y)\right),
\end{gathered}
$$

where $a_{k, l}, A_{k, l}, B_{k, l}$ are unknowns to be determined.

The motivation in [24] for choosing $W$ of the form (33) is precisely the closeness of the family of functions

$$
\left\{c^{l} Q_{c}^{k}, c^{l}\left(Q_{c}^{k}\right)^{\prime}, k \geq 1, l \geq 0\right\}
$$

under multiplication and differentiation, due to the specific form of the equation of $Q_{c}$ (see Lemma 2.1 in [24]). In the case of equation (28), for a general nonlinearity this structure is preserved up to a lower order term (see Lemma A.2).

We want to measure the size of the error produced by inserting $\tilde{u}$ as defined in (32)-(33) in the equation (28). For this, let

$$
S[\tilde{u}](t, x):=\tilde{u}_{t}+\left(\tilde{u}_{x x}-\tilde{u}+f(\tilde{u})\right)_{x} .
$$

Our first result in the above direction is the following

Proposition 2.2 (Decomposition of $S(\tilde{u}))$. Let

$$
\mathcal{L} w:=-w_{y y}+w-f^{\prime}(Q) w .
$$


Then,

$$
\begin{aligned}
S[\tilde{u}](t, x)= & \sum_{(k, l) \in \Sigma_{m}} c^{l} Q_{c}^{k}\left(y_{c}\right)\left[a_{k, l}(-3 Q+2 f(Q))^{\prime}(y)-\left(\mathcal{L} A_{k, l}\right)^{\prime}(y)+F_{k, l}(y)\right] \\
& +\sum_{(k, l) \in \Sigma_{m}} c^{l}\left(Q_{c}^{k}\right)^{\prime}\left(y_{c}\right)\left[a_{k, l}\left(-3 Q^{\prime \prime}\right)(y)+\left(3 A_{k, l}^{\prime \prime}+f^{\prime}(Q) A_{k, l}\right)(y)-\left(\mathcal{L} B_{k, l}\right)^{\prime}(y)+G_{k, l}(y)\right] \\
& +\mathcal{E}(t, x)
\end{aligned}
$$

where $F_{k, l}, G_{k, l}$ and $\mathcal{E}$ satisfy, for any $(k, l) \in \Sigma_{m}$,

(i) Dependence property of $F_{k, l}$ and $G_{k, l}$ : The expressions of $F_{k, l}$ and $G_{k, l}$ depend only on $\left(a_{k^{\prime}, l^{\prime}}\right),\left(A_{k^{\prime}, l^{\prime}}\right),\left(B_{k^{\prime}, l^{\prime}}\right)$ for $\left(k^{\prime}, l^{\prime}\right)<(k, l)$.

(ii) Parity property of $F_{k, l}$ and $G_{k, l}$ : Assume that for any $\left(k^{\prime}, l^{\prime}\right)$ such that $\left(k^{\prime}, l^{\prime}\right)<(k, l)$ $A_{k^{\prime}, l^{\prime}}$ is even and $B_{k^{\prime}, l^{\prime}}$ is odd, then $F_{k, l}$ is odd and $G_{k, l}$ is even.

Moreover, $F_{1,0}=\left(f^{\prime}(Q)\right)^{\prime}$ and $G_{1,0}=f^{\prime}(Q)$, and higher order terms are given in Appendix $A$.

(iii) Estimate on $\mathcal{E}:$ Assume both $\left(A_{k, l}\right)$ and $\left(B_{k, l}\right)$ bounded, and $\left(A_{k, l}^{\prime}\right),\left(B_{k, l}^{\prime}\right) \in \mathcal{Y}$ for $(k, l) \in$ $\Sigma_{m}$. Then there exists $\kappa>0$ such that for all $j=0,1,2$, and for every $(t, x) \in\left[-T_{c}, T_{c}\right] \times \mathbb{R}$,

$$
\left|\partial_{x}^{j} \mathcal{E}(t, x)\right| \leq \kappa c^{m-1} Q_{c}\left(y_{c}\right) .
$$

Remark 2.2. Note that $(\mathcal{L} w)_{y}$, as defined in (36), represents the linear operator associated to the gKdV equation (28). Thus, the expression for $S[\tilde{u}]$ above stated can be seen as a generalization of the linearized $\mathrm{gKdV}$ equation, with the addition of some correction terms.

Proof. We postpone the proof of the Proposition 2.2. merely calculative, to Appendix A We note that this Proposition has been already stated in [25, but here we will need an improved version, describing explicitly every term $F_{k, l}, G_{k, l}$ up to a fixed high order. For the details, see Appendix $\mathrm{A}$

Note that if we want to improve the approximation $\tilde{u}$, the unknown functions $A_{k, l}$ and $B_{k, l}$ for a fixed $(k, l)$ must be chosen satisfying a sort of modified linear gKdV system where the source terms are composed of preceding, well-known, $A_{k^{\prime}, l^{\prime}}$ and $B_{k^{\prime}, l^{\prime}}$ functions. Indeed, if we choose (formally) $A_{k, l}$ and $B_{k, l}$ such that for any $(k, l) \in \Sigma_{m}$

$$
\left(\Omega_{k, l}\right) \quad\left\{\begin{array}{l}
\left(\mathcal{L} A_{k, l}\right)^{\prime}+a_{k, l}(3 Q-2 f(Q))^{\prime}=F_{k, l}, \\
\left(\mathcal{L} B_{k, l}\right)^{\prime}+3 a_{k, l} Q^{\prime \prime}-3 A_{k, l}^{\prime \prime}-f^{\prime}(Q) A_{k, l}=G_{k, l},
\end{array}\right.
$$

then the error term will be reduced to the quantity

$$
S[\tilde{u}]=\mathcal{E}(t, x) .
$$

Of course the solvability theory for the linear systems $\left(\Omega_{k, l}\right)$ and the measure of this error term must be stated in a rigorous form. This will be established in the following section.

2.2. Resolution of linear systems $\left(\Omega_{k, l}\right)$. First, we recall some preliminary notation and results from [24. We denote by $\mathcal{Y}$ the set of $C^{\infty}$ functions $f$ such that

$$
\forall j \in \mathbb{N}, \exists K_{j}, r_{j}>0, \forall x \in \mathbb{R}, \quad\left|f^{(j)}(x)\right| \leq K_{j}(1+|x|)^{r_{j}} e^{-|x|} .
$$

We recall some well-known results concerning a resonance function and the operator $\mathcal{L}$.

Claim 1 (25]). The function $\varphi(x)=-\frac{Q^{\prime}(x)}{Q(x)}$ is odd and satisfies:

(i) $\lim _{x \rightarrow-\infty} \varphi(x)=-1 ; \lim _{x \rightarrow+\infty} \varphi(x)=1$;

(ii) $\forall x \in \mathbb{R},\left|\varphi^{\prime}(x)\right|+\left|\varphi^{\prime \prime}(x)\right|+\left|\varphi^{(3)}(x)\right| \leq C e^{-|x|}$.

(iii) $\varphi^{\prime} \in \mathcal{Y},\left(1-\varphi^{2}\right) \in \mathcal{Y}$.

Lemma 2.3 (Properties of $\mathcal{L}$, see [25]). The operator $\mathcal{L}$ defined in $L^{2}(\mathbb{R})$ by (36) has domain $H^{2}(\mathbb{R})$, is self-adjoint and satisfies the following properties:

(i) There exist a unique $\lambda_{0}>0, \chi_{0} \in H^{1}(\mathbb{R}), \chi_{0}>0$ such that $\mathcal{L} \chi_{0}=-\lambda_{0} \chi_{0}$. 
(ii) The kernel of $\mathcal{L}$ is $\left\{\lambda Q^{\prime}, \lambda \in \mathbb{R}\right\}$. Let $\Lambda Q:=\frac{d}{d c} Q_{c \mid c=1}$, then $\mathcal{L}(\Lambda Q)=-Q$.

(iii) (Inverse) For all $h \in L^{2}(\mathbb{R})$ such that $\int_{\mathbb{R}} h Q^{\prime}=0$, there exists a unique $\widetilde{h} \in H^{2}(\mathbb{R})$ such that $\int_{\mathbb{R}} \widetilde{h} Q^{\prime}=0$ and $\mathcal{L} \widetilde{h}=h$; moreover, if $h$ is even (resp. odd), then $\widetilde{h}$ is even (resp. odd).

(iv) For $h \in H^{2}(\mathbb{R}), \mathcal{L} h \in \mathcal{Y}$ implies $h \in \mathcal{Y}$.

(v) (Coercivity) If $\frac{d}{d \widetilde{c}} \int_{\mathbb{R}} Q_{\widetilde{c} \mid \widetilde{c}=c}^{2}>0$ then there exists $\lambda_{c}>0$ such that if

$$
\int_{\mathbb{R}} w Q_{c}=\int_{\mathbb{R}} w Q_{c}^{\prime}=0 \quad \text { then } \quad \int_{\mathbb{R}}\left(w_{x}^{2}+c w^{2}-f^{\prime}\left(Q_{c}\right) w^{2}\right) \geq \lambda_{c} \int_{\mathbb{R}} w^{2} .
$$

(vi) There exist unique even solutions $P$ and $\bar{P}$ of the ordinary differential equations

$$
\begin{gathered}
\mathcal{L} P=3 Q^{\prime \prime}+f^{\prime}(Q) Q, \quad P \in \mathcal{Y}, \\
\mathcal{L} \bar{P}=f^{\prime}(Q), \quad \bar{P} \in \mathcal{Y} . \\
\text { Moreover, } P:=-\left(x Q^{\prime}+\Lambda Q+Q\right) .
\end{gathered}
$$

Remark 2.3. Item (vi) from above Lemma is new; the proof follows directly from (ii), (iii) and (iv). On the other hand, for general nonlinearities $\bar{P}$ is not explicit.

2.2.1. Existence theory for a model problem. We recall that linear systems $\left(\Omega_{k, l}\right)$ are very similar and then proving existence reduces to prove the result for a model problem. This idea comes from [24, but we will need a simplified version, from [26].

Proposition 2.4 (Existence for a model problem, see [25]). Let $F \in \mathcal{Y}$, odd, and $G \in \mathcal{Y}$, even. Let $\gamma, \kappa \in \mathbb{R}$. Then, there exist $a, b \in \mathbb{R}, \widetilde{A} \in \mathcal{Y}$ even, and $\widetilde{B} \in \mathcal{Y}$ odd, such that

$$
A=\widetilde{A}+\gamma, \quad \text { and } \quad B=\widetilde{B}+b \varphi+\kappa Q^{\prime}
$$

satisfy

$$
(\Omega) \quad\left\{\begin{array}{l}
(\mathcal{L} A)^{\prime}+a(3 Q-2 f(Q))^{\prime}=F \\
(\mathcal{L} B)^{\prime}+3 a Q^{\prime \prime}-3 A^{\prime \prime}-f^{\prime}(Q) A=G
\end{array}\right.
$$

Moreover,

$$
a=\frac{-1}{\int_{\mathbb{R}} \Lambda Q Q}\left\{\gamma \int_{\mathbb{R}} P+\int_{\mathbb{R}} G Q-\int_{\mathbb{R}} F \int_{0}^{x} P\right\}
$$

and

$$
b=\frac{1}{2}\left[\gamma \int_{\mathbb{R}} \bar{P}+a \int_{\mathbb{R}} \Lambda Q-\int_{\mathbb{R}} F \int_{0}^{x} \bar{P}+\int_{\mathbb{R}} G\right] .
$$

Proof. We give a sketch of the proof for the sake of completeness. The original result comes from [25, and here it is even simpler since we deal only with $F, G \in \mathcal{Y}$.

Set $A:=\widetilde{A}+\gamma, B:=\widetilde{B}+b \varphi$, where $\gamma$ is given, while $b$ is a parameter to be found. Since $(\mathcal{L} 1)^{\prime}=(1-f(Q))^{\prime}=-(f(Q))^{\prime}$, we obtain the following system for $\widetilde{A}, \widetilde{B}$ :

$$
\left\{\begin{array}{l}
(\mathcal{L} \widetilde{A})^{\prime}+a(3 Q-2 f(Q))^{\prime}=F+\gamma(f(Q))^{\prime}, \\
(\mathcal{L} \widetilde{B})^{\prime}+3 a Q^{\prime \prime}-3 \widetilde{A}^{\prime \prime}-f^{\prime}(Q) \widetilde{A}=G+\gamma f^{\prime}(Q)-b(\mathcal{L} \varphi)^{\prime} .
\end{array}\right.
$$

Note that $F \in \mathcal{Y}$ is odd, therefore $\mathcal{H}(x)=\int_{-\infty}^{x} F(z) d z+\gamma f(Q)$ belong to $\mathcal{Y}$ and is even. By integration of the first line, we are reduced to solve

$$
\left\{\begin{array}{l}
\mathcal{L} \widetilde{A}+a(3 Q-2 f(Q))=\mathcal{H} \\
(\mathcal{L} \widetilde{B})^{\prime}+3 a Q^{\prime \prime}-3 \widetilde{A}^{\prime \prime}-f^{\prime}(Q) \widetilde{A}=G+\gamma f^{\prime}(Q)-b(\mathcal{L} \varphi)^{\prime}
\end{array}\right.
$$

Since $\int_{\mathbb{R}} \mathcal{H} Q^{\prime}=0$ (by parity) and $\mathcal{H} \in \mathcal{Y}$, by Lemma 2.3, there exists $\bar{H} \in \mathcal{Y}$, even, such that $\mathcal{L} \bar{H}=\mathcal{H}$.

Define $\hat{P}$ to be the unique even solution of

$$
\mathcal{L} \hat{P}=3 Q-2 f(Q), \quad \hat{P} \in \mathcal{Y} .
$$


Indeed, $\hat{P}$ has an explicit formula

$$
\hat{P}=-\left(x Q^{\prime}+\Lambda Q\right), \quad \text { with } \mathcal{L}(\Lambda Q)=-Q .
$$

It follows that $\widetilde{A}:=-a \hat{P}+\bar{H}$ is even, belongs to $\mathcal{Y}$ and solves the first line of the previous system. Note that at this stage, the parameters $a$ and $b$ are still free.

Now, we only need to find $\widetilde{B} \in \mathcal{Y}$, odd, such that $(\mathcal{L} \widetilde{B})^{\prime}=-a Z_{0}+D-b(\mathcal{L} \varphi)^{\prime}$, where

$$
D:=3 \bar{H}^{\prime \prime}+f^{\prime}(Q) \bar{H}+G+\gamma f^{\prime}(Q) \in \mathcal{Y}, \text { even, } Z_{0}:=3 Q^{\prime \prime}+3 \hat{P}^{\prime \prime}+f^{\prime}(Q) \hat{P} \in \mathcal{Y} \text {, even. }
$$

Let

$$
E:=\int_{0}^{x}\left(D-a Z_{0}\right)(z) d z-b \mathcal{L} \varphi
$$

This function a priori is in $L^{\infty}(\mathbb{R})$, independent of $a, b$.

Claim 2. There exist numbers $a$ and $b$ such that $E \in \mathcal{Y}$ and $\int_{\mathbb{R}} E Q^{\prime}=0$.

Assuming Claim 2, we fix $a, b$ so that $E \in \mathcal{Y}$ and $\int_{\mathbb{R}} E Q^{\prime}=0$. It follows from Lemma 2.3 that there exists $\widetilde{B} \in \mathcal{Y}$, odd, such that $\mathcal{L} \widetilde{B}=E$. The final solution is then given by $A:=\widetilde{A}+\gamma$ and $B:=\widetilde{B}+b \varphi+\kappa Q^{\prime}$, where $\kappa$ is a free parameter, because $\mathcal{L} Q^{\prime}=0$ (see Lemma 2.3, (ii)).

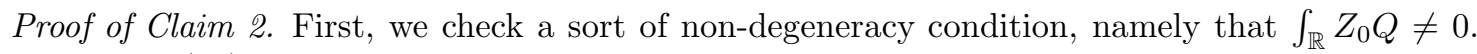
Indeed, by (38)

$$
\int_{\mathbb{R}} Z_{0} Q=-3 \int_{\mathbb{R}} Q^{\prime 2}+\int_{\mathbb{R}} \mathcal{L} P \hat{P}=-3 \int_{\mathbb{R}} Q^{\prime 2}+\int_{\mathbb{R}} P(3 Q-2 f(Q)) .
$$

We recall now the following auxiliary result.

Claim 3 (25, Claim 2.2). We have

$$
3 \int_{\mathbb{R}} Q^{\prime 2}-\int_{\mathbb{R}}(3 Q-2 f(Q)) P=\int_{\mathbb{R}} \Lambda Q Q \neq 0 .
$$

Remark 2.4. Indeed,

$$
\int_{\mathbb{R}} \Lambda Q Q=\left.\frac{1}{2} \partial_{c} \int_{\mathbb{R}} Q_{c}^{2}\right|_{c=1}>0
$$

thanks to (12) provided $\varepsilon$ small enough (independent of $c$ ).

Let us continue with the proof of Claim 2, By the preceding result, it suffices to choose $a:=\frac{\int_{\mathbb{R}} D Q}{\int_{\mathbb{R}} Z_{0} Q}$, and $b:=\int_{0}^{+\infty}\left(D-a Z_{0}\right)(z) d z\left(\right.$ note that $\left.\lim _{ \pm \infty} \mathcal{L} \varphi=\lim _{ \pm \infty} \varphi= \pm 1\right)$. This finishes the proof of Claim 2 .

We return to the proof of Proposition 2.4 Now we find the constants $a$ and $b$ in terms of known quantities in $(\Omega)$. First, we multiply the equation of $B$ by $Q$ and use $\mathcal{L} Q^{\prime}=0$. We get

$$
\begin{aligned}
-3 a \int_{\mathbb{R}} Q^{\prime 2} & =\int_{\mathbb{R}}\left(3 Q^{\prime \prime}+f^{\prime}(Q) Q\right) A+\int_{\mathbb{R}} G Q \\
& =\int_{\mathbb{R}}(\mathcal{L} A) P+\int_{\mathbb{R}} G Q .
\end{aligned}
$$

Second, we multiply the equation of $A$ by $\int_{0}^{x} P(s) d s$. We obtain

$$
\begin{aligned}
\int_{\mathbb{R}}(\mathcal{L} A)^{\prime} \int_{0}^{x} P & =-\int_{\mathbb{R}}(\mathcal{L} A) P+\gamma \int_{\mathbb{R}} P \\
& =a \int_{\mathbb{R}}(3 Q-2 f(Q)) P+\int_{\mathbb{R}} F \int_{0}^{x} P .
\end{aligned}
$$

Thus, combining the two identities, we get:

$$
-a\left\{3 \int_{\mathbb{R}} Q^{\prime 2}-\int_{\mathbb{R}}(3 Q-2 f(Q)) P\right\}=\gamma \int_{\mathbb{R}} P+\int_{\mathbb{R}} G Q-\int_{\mathbb{R}} F \int_{0}^{x} P .
$$


and the expression for $a$ follows from Claim 3

To find out $b$, we integrate the equation for $B$ in $(\Omega)$ over $\mathbb{R}$ to obtain

$$
2 b=\int_{\mathbb{R}} f^{\prime}(Q) A+\int_{\mathbb{R}} G .
$$

Now we consider $\bar{P}$ the function defined in (39). We multiply the equation for $A$ by $\int_{0}^{x} \bar{P}(s) d s$ and then we integrate. We get

$$
\int_{\mathbb{R}} f^{\prime}(Q) A=\gamma \int_{\mathbb{R}} \bar{P}-a \int_{\mathbb{R}} \bar{P}(3 Q-2 f(Q))-\int_{\mathbb{R}} F \int_{0}^{x} \bar{P} .
$$

Now, note that

$$
\int_{\mathbb{R}} \bar{P}(3 Q-2 f(Q))=\int_{\mathbb{R}} \mathcal{L} \hat{P} \bar{P}=\int_{\mathbb{R}} \hat{P} f^{\prime}(Q)=\int_{\mathbb{R}} \hat{P}(1-\mathcal{L} 1)=\int_{\mathbb{R}} \hat{P}-\int_{\mathbb{R}}(3 Q-2 f(Q)) .
$$

From (42) we replace the explicit value of $\hat{P}$ and we use the equation satisfied by $Q$, namely $Q^{\prime \prime}-Q+f(Q)=0$, to obtain

$$
\int_{\mathbb{R}} \bar{P}(3 Q-2 f(Q))=-\int_{\mathbb{R}} \Lambda Q .
$$

With $a$ previously known we replace this quantity in (43) to obtain (41). This finishes the proof.

We have now a good solvability theory for the linear systems $\left(\Omega_{k, l}\right)$, that avoids the emergency of linearly growing solutions at this order. As an example, the general theory constructed in [24] for the quartic KdV equation deals with possibly growing solutions, see 24] Proposition 2.3.

Here, for each system $\left(\Omega_{k, l}\right),(k, l) \in \Sigma_{m}$, we will look for solutions such that

$$
A_{k, l}=\widetilde{A}_{k, l}+\gamma_{k, l}, \quad B_{k, l}=\widetilde{B}_{k, l}+b_{k, l} \varphi+\kappa_{k, l} Q^{\prime}, \quad a_{k, l}, b_{k, l}, \kappa_{k, l} \in \mathbb{R}
$$

where $\widetilde{A}_{k, l} \in \mathcal{Y}$ is even and $\widetilde{B}_{k, l} \in \mathcal{Y}$ is odd. (see Proposition 2.10 for a justification of this choice).

This election will have several good properties, but we will emphasize a crucial one. Let $(k, l) \in \Sigma_{m}$ fixed. We say that $(k, l)$ satisfies the (IP) property (IP = important property) if and only if

$$
\text { (IP) }\left\{\begin{array}{l}
\text { Any derivative of } A_{k, l} \text { or } B_{k, l} \text { is a localized } \mathcal{Y} \text {-function. } \\
\text { Moreover, for }(k, l)=(1,0) \text { we have } A_{1,0} \in \mathcal{Y} \text {. }
\end{array}\right.
$$

This property, although depending on the specific pair $(k, l)$, will be useful to quickly discard localized terms composing $F_{k, l}, G_{k, l}$, and seeing essentially the bounded but non localized terms. Indeed, note that thanks to Claim 1 any solution as in (44) satisfies this property. For the details, see Appendix $\mathrm{A}$.

We start by solving the first system.

2.2.2. Resolution of the system $\left(\Omega_{1,0}\right)$. From Proposition 2.2 (ii) the system $\left(\Omega_{1,0}\right)$ is given by

$$
\begin{aligned}
& \left(\mathcal{L} A_{1,0}\right)^{\prime}=-a_{1,0}(3 Q-2 f(Q))^{\prime}+\left(f^{\prime}(Q)\right)^{\prime}, \\
& \left(\mathcal{L} B_{1,0}\right)^{\prime}=3 A_{1,0}^{\prime \prime}+f^{\prime}(Q) A_{1,0}-3 a_{1,0} Q^{\prime \prime}+f^{\prime}(Q)
\end{aligned}
$$

This first system is easily solvable, as shows the following

Lemma 2.5 (Resolution of $\left.\left(\Omega_{1,0}\right)\right)$. There exists a solution $\left(A_{1,0}, B_{1,0}, a_{1,0}\right)$ of 455 - 46) of the form (44) and such that $A_{1,0} \in \mathcal{Y}$ is even (and $\gamma_{1,0}=0$ ), $B_{1,0}$ is odd and $a_{1,0}, b_{1,0}$ are given by the formulae

$$
a_{1,0}=\frac{\int_{\mathbb{R}} \Lambda Q}{\int_{\mathbb{R}} \Lambda Q Q}, \quad b_{1,0}=\frac{1}{2} a_{1,0} \int_{\mathbb{R}} \Lambda Q+\frac{1}{2} \int_{\mathbb{R}} \bar{P} .
$$

Moreover, $A_{1,0}$ is given by

$$
A_{1,0}=\bar{P}-a_{1,0} \hat{P} .
$$


(cf. (45), (42) and (39)). Finally, we choose $B_{1,0}$ such that $\int_{\mathbb{R}} Q^{\prime} B_{1,0}=0$.

Remark 2.5. Note that from the value of $\hat{P}=-\left(x Q^{\prime}+\Lambda Q\right)$ and (48) we get

$$
b_{1,0}=\frac{1}{2}\left[a_{1,0} \int_{\mathbb{R}} Q+\int_{\mathbb{R}} A_{1,0}\right] .
$$

Proof. Note that both $\left(f^{\prime}(Q)\right)^{\prime}$ and $f^{\prime}(Q)$ are odd and even $\mathcal{Y}$-functions respectively, so thanks to Proposition 2.4, a solution with the desired properties does exist. We will chose $\gamma_{1,0}:=0$. The value of $a_{1,0}$ and $b_{1,0}$ comes from (40)-(41), after some simple computations. These computations have been carried out in [25], but by completeness we rewrite them. Indeed, note that we only need to verify that

$$
\int_{\mathbb{R}} f^{\prime}(Q)(Q+P)=-\int_{\mathbb{R}} \Lambda Q
$$

In fact, from (38), the explicit value of $P$ and Claim 2.3 (ii), we have

$$
\int_{\mathbb{R}} f^{\prime}(Q)(Q+P)=-\int_{\mathbb{R}} f^{\prime}(Q)\left(x Q^{\prime}+\Lambda Q\right)=\int_{\mathbb{R}} f(Q)-\int_{\mathbb{R}}(1-\mathcal{L} 1) \Lambda Q=\int_{\mathbb{R}}(f(Q)-Q)-\int_{\mathbb{R}} \Lambda Q,
$$

but $f(Q)-Q=-Q^{\prime \prime}$, so we are done.

On the other hand, note that $\mathcal{L}(1+\bar{P})=1$, thus

$$
\int_{\mathbb{R}} f^{\prime}(Q)(\bar{P}+1)=\int_{\mathbb{R}} \mathcal{L} \bar{P}(1+\bar{P})=\int_{\mathbb{R}} \bar{P} 1 .
$$

This give finally the expected value of $b_{1,0}$.

Finally, the constant $\kappa_{1,0}$ in the expression of $B_{1,0}$ is a free parameter that we will fix such that $\int_{\mathbb{R}} B_{1,0} Q^{\prime}=0$ for convenience in some future computations (see Proposition 2.4 and (185) in Appendix (B). We have

$$
0=\int_{\mathbb{R}} Q^{\prime} B_{1,0}=\int_{\mathbb{R}} \widetilde{B}_{1,0} Q^{\prime}+b_{1,0} \int_{\mathbb{R}} \varphi Q^{\prime}+\kappa_{1,0} \int_{\mathbb{R}} Q^{\prime 2} .
$$

where we can obtain $\kappa_{1,0}$.

2.2.3. Resolution of the system $\left(\Omega_{2,0}\right)$. From Proposition A.1 (iii) in Appendix A, the system $\left(\Omega_{2,0}\right)$ is given by

$$
\begin{aligned}
& \left(\mathcal{L} A_{2,0}\right)^{\prime}=a_{2,0}(3 Q-2 f(Q))^{\prime}+F_{2,0}, \\
& \left(\mathcal{L} B_{2,0}\right)^{\prime}=3 A_{2,0}^{\prime \prime}+f^{\prime}(Q) A_{2,0}-3 a_{2,0} Q^{\prime \prime}+G_{2,0}
\end{aligned}
$$

where the source terms are given by

(1) Case $m=2$,

$$
\begin{aligned}
F_{2,0}=- & \left(3 A_{1,0}^{\prime}+3 B_{1,0}^{\prime \prime}+f^{\prime}(Q) B_{1,0}\right)+\frac{1}{2}\left(f^{\prime \prime}(Q)\left(2 A_{1,0}+A_{1,0}^{2}\right)\right)^{\prime} \\
& -a_{1,0}\left(3 A_{1,0}^{\prime \prime}-Q+f^{\prime}(Q)\left(1+A_{1,0}\right)\right)^{\prime}+3 a_{1,0}^{2} Q^{(3)}+\frac{1}{2}\left(f^{\prime \prime}(Q)-2\right)^{\prime},
\end{aligned}
$$

and

$$
\begin{aligned}
G_{2,0}= & \frac{1}{2}\left(f^{\prime \prime}(Q)-2\right)-\left(A_{1,0}+3 B_{1,0}^{\prime}\right)+\frac{1}{2} f^{\prime \prime}(Q)\left(2 A_{1,0}+A_{1,0}^{2}\right)+\frac{3}{2} a_{1,0}^{2} Q^{\prime \prime} \\
& -\frac{1}{2} a_{1,0}\left(9 A_{1,0}^{\prime}+3 B_{1,0}^{\prime \prime}+f^{\prime}(Q) B_{1,0}\right)^{\prime}+\frac{1}{2}\left(f^{\prime \prime}(Q)\left(B_{1,0}+A_{1,0} B_{1,0}\right)\right)^{\prime} .
\end{aligned}
$$

(2) Case $m=3$,

$$
F_{2,0}=\left(\frac{1}{2} f^{\prime \prime}(Q)\left(1+A_{1,0}\right)^{2}\right)^{\prime}+3 a_{1,0}^{2} Q^{(3)}-a_{1,0}\left(f^{\prime}(Q)+3 A_{1,0}^{\prime \prime}+f^{\prime}(Q) A_{1,0}\right)^{\prime}
$$


and

$$
\begin{aligned}
G_{2,0}= & \frac{1}{2} f^{\prime \prime}(Q)\left(1+A_{1,0}\right)^{2}+\frac{3}{2} a_{1,0}^{2} Q^{\prime \prime}-\frac{1}{2} a_{1,0}\left(9 A_{1,0}^{\prime}+3 B_{1,0}^{\prime \prime}+f^{\prime}(Q) B_{1,0}\right)^{\prime} \\
& +\frac{1}{2}\left(f^{\prime \prime}(Q)\left(1+A_{1,0}\right) B_{1,0}\right)^{\prime} .
\end{aligned}
$$

Proposition 2.6 (Resolution of $\left(\Omega_{2,0}\right)$ ). Let $f$ be as in 22). There exists a constant $\varepsilon_{0}>0$ not depending on $c$ such that the following holds.

(1) (Case $m=2)$ There exists a solution $\left(A_{2,0}, B_{2,0}, a_{2,0}\right)$ of $\left(\Omega_{2,0}\right)$ satisfying (44) and such that

$$
\begin{gathered}
\lim _{+\infty} A_{2,0}=-\frac{1}{2} b_{1,0}^{2}=\gamma_{2,0}, \quad A_{2,0}-\gamma_{2,0} \in \mathcal{Y}, \\
\lim _{+\infty} B_{2,0}=b_{2,0}, \quad B_{2,0}-b_{2,0} \varphi \in \mathcal{Y},
\end{gathered}
$$

but for all $|\varepsilon| \in\left(0, \varepsilon_{0}\right)$

$$
d(\varepsilon):=b_{2,0}(f)+\frac{1}{6} b_{1,0}^{3}(f)=c_{2, p} \varepsilon+o(\varepsilon), \quad \text { with } \quad c_{2, p} \neq 0 \text { for all } p \geq 4 .
$$

(2) (Case $m=3$ ) There exists a solution $\left(A_{2,0}, B_{2,0}, a_{2,0}\right)$ of $\left(\Omega_{2,0}\right)$ such that $A_{2,0} \in \mathcal{Y}$ is even, $B_{2,0}$ is bounded, odd and

$$
\lim _{+\infty} B_{2,0}=b_{2,0}, \quad B_{2,0}-b_{2,0} \varphi \in \mathcal{Y}
$$

but for all $|\varepsilon| \in\left(0, \varepsilon_{0}\right)$,

$$
d(\varepsilon):=b_{2,0}(f)=c_{3, p} \varepsilon+o(\varepsilon), \quad \text { and } \quad c_{3, p} \neq 0 \text { for all } p \geq 4 .
$$

Moreover, in both cases the solution found satisfies (IP).

Remark 2.6. Note that in the case $m=2$, one has $c_{2, p}=0$ for $p=3$ (see (60) ). This cancelation is consequence of the complete integrability of the Gardner equation.

Proof. Note that in both cases, $m=2$ and $m=3$ the source terms $F_{2,0}, G_{2,0}$ belongs to $\mathcal{Y}$, with the former being an odd function and the last one being even. Thus the existence of solutions to (50)-(51) with the desired properties follows directly from Proposition 2.4 above.

In particular we will choose $\gamma_{2,0}:=-\frac{1}{2} b_{1,0}^{2}$ for the quadratic case and $\gamma_{2,0}:=0$ in the cubic one.

Let us now check that, being fixed $\gamma_{1,0}, a_{1,0}, b_{1,0}$ and $\gamma_{2,0}$, the value of $a_{2,0}$ and $b_{2,0}$ is uniquely determined. Indeed, from (40)-(41)

$$
a_{2,0}=-\frac{1}{\int_{\mathbb{R}} \Lambda Q Q}\left[\gamma_{2,0} \int_{\mathbb{R}} P+\int_{\mathbb{R}} G_{2,0} Q-\int_{\mathbb{R}} F_{2,0} \int_{0}^{x} P\right],
$$

and

$$
b_{2,0}=\frac{1}{2}\left[\gamma_{2,0} \int_{\mathbb{R}} \bar{P}+a_{2,0} \int_{\mathbb{R}} \Lambda Q-\int_{\mathbb{R}} F_{2,0} \int_{0}^{x} \bar{P}+\int_{\mathbb{R}} G_{2,0}\right] .
$$

We claim (56) and (57) with

$$
c_{2, p}:=-\left[\frac{(p-3)(2 p-1)\left(24-23 p+3 p^{2}+2 p^{3}\right)}{36\left(p^{2}-1\right)(p-2)}\right] \int_{\mathbb{R}}\left[\frac{3}{2 \cosh ^{2}\left(\frac{1}{2} x\right)}\right]^{p},
$$

and

$$
c_{3, p}:=-\left[\frac{(p-1)(p-3)\left(p^{2}-3 p+8\right)}{8(p-2)(p+1)}\right] \int_{\mathbb{R}}\left[\frac{\sqrt{2}}{\cosh x}\right]^{p} .
$$

The end of the proof of (56)-(57), and (60)-(61) is a lengthy but straightforward computation. For the sake of continuity we postpose the proof to Appendix B.

Remark 2.7. An explicit expression for $d(\varepsilon)$ for any nonlinearity has escaped to us (see Claim 11), and we only have in our hands an asymptotic expression for small values of $\varepsilon$. We believe, however, that it may exist a -necessarily- large $\varepsilon_{0}$ for which $d\left(\varepsilon_{0}\right)=0$, and even more, a pure 2-soliton solution may exist at any order. 
Remark 2.8. The expressions (56)-(57) above say roughly speaking that the second order linear system $\left(\Omega_{2,0}\right)$ has a solution that does not obey (at third order derivatives) the Taylor expansion of a small soliton shifted. Indeed,

$$
Q_{c}\left(y_{c}+b_{1,0} \varphi\right) \sim Q_{c}\left(y_{c}\right)+b_{1,0} \varphi Q_{c}^{\prime}\left(y_{c}\right)+\frac{1}{2} b_{1,0}^{2} Q_{c}^{\prime \prime}\left(y_{c}\right)+\frac{1}{6} b_{1,0}^{3} Q_{c}^{(3)}\left(y_{c}\right) .
$$

Note that (cf. (128) and (129))

$$
Q_{c}^{\prime \prime}\left(y_{c}\right) \sim c Q_{c}\left(y_{c}\right)-Q_{c}^{m}\left(y_{c}\right), \quad Q_{c}^{(3)}\left(y_{c}\right) \sim c Q_{c}^{\prime}\left(y_{c}\right)-\left(Q_{c}^{m}\right)^{\prime}\left(y_{c}\right),
$$

and thus for a perfect collision we should have $b_{2,0}=-\frac{1}{6} b_{1,0}^{3}$ for $m=2$ and $b_{2,0}=0$ for $m=3$, as in the integrable cases. This formal discussion will be justified in the proof of Proposition 2.10,

2.2.4. Resolution of system $\left(\Omega_{1,1}\right)$, cases $m=2,3$. Now we consider the first mixed system, $\left(\Omega_{1,1}\right)$. Note that this system has a different order depending on the power of leading nonlinearity: for $m=2, c Q_{c}$ is of quadratic order in $Q_{c}$, meanwhile, in the cubic one, $c Q_{c}$ is a term of cubic order.

From Proposition A.1 the system $\left(\Omega_{1,1}\right)$ is given by

$$
\begin{aligned}
& \left(\mathcal{L} A_{1,1}\right)^{\prime}=a_{1,1}(3 Q-2 f(Q))^{\prime}+\left(3 A_{1,0}^{\prime}+3 B_{1,0}^{\prime \prime}+f^{\prime}(Q) B_{1,0}\right), \\
& \left(\mathcal{L} B_{1,1}\right)^{\prime}=3 A_{1,1}^{\prime \prime}+f^{\prime}(Q) A_{1,1}-3 a_{1,1} Q^{\prime \prime}+3 B_{1,0}^{\prime}
\end{aligned}
$$

For this system, we recall its source terms

$$
F_{1,1}:=3 A_{1,0}^{\prime}+3 B_{1,0}^{\prime \prime}+f^{\prime}(Q) B_{1,0}, \quad G_{1,1}:=3 B_{1,0}^{\prime} .
$$

Note that as $(k, l)=(1,0)$ satisfies the (IP) property, we have both $F_{1,1}, G_{1,1} \in \mathcal{Y}$.

Lemma 2.7 (Resolution of $\left.\left(\Omega_{1,1}\right), m=2,3\right)$. There exists a solution $\left(A_{1,1}, B_{1,1}, a_{1,1}\right)$ of $\left(\Omega_{1,1}\right)$ such that $A_{1,1}$ is even, $B_{1,1}$ is odd and

$$
\begin{gathered}
\lim _{+\infty} A_{1,1}=\gamma_{1,1}:=\frac{1}{2} b_{1,0}^{2}, \quad A_{1,1}-\gamma_{1,1} \in \mathcal{Y}, \\
\lim _{+\infty} B_{1,1}=b_{1,1}, \quad B_{1,1}-b_{1,1} \varphi \in \mathcal{Y} .
\end{gathered}
$$

Besides, this solution implies that (IP) holds for $(k, l)=(1,1)$.

Proof. From Proposition 2.2, it is clear that $F_{1,1}$ and $G_{1,1}$ given in (64) satisfy the assumptions of Proposition 2.4. The choice of $\gamma_{1,1}$ will be justified in Proposition 2.10 In the rest of this paper, we will not need the expression of $b_{1,1}$ (note that it would be possible to compute it as in the proof of Proposition 2.67).

2.2.5. Resolution of high order systems, quadratic case. From now on, we consider the triplet

$$
\left(A_{k, l}, B_{k, l}, a_{k, l}\right)
$$

defined for all $(k, l) \in \Sigma_{m}, 1 \leq k+l \leq 2$ in Lemma 2.5. Proposition 2.6 and Lemma 2.7. We now solve the systems $\left(\Omega_{k, l}\right)$ for $k+l=3$. Denote $\delta_{33}:=1$ and $\delta_{p 3}:=0$ for $p \geq 4$.

Lemma 2.8 (Resolution of $\left(\Omega_{k, l}\right)$ for $k+l=3$ and $m=2$ ). For all $(k, l) \in \Sigma_{2}$ such that $k+l=3$, $F_{k, l}$ is odd and $G_{k, l}$ even; both are in the class $\mathcal{Y}$, and there exists a solution $\left(A_{k, l}, B_{k, l}, a_{k, l}\right)$ of $\left(\Omega_{k, l}\right)$ such that $A_{k, l}$ is even, $B_{k, l}$ is odd and

$$
\begin{aligned}
& \lim _{+\infty} A_{k, l}=\gamma_{k, l}, \quad A_{k, l}-\gamma_{k, l} \in \mathcal{Y}, \\
& \lim _{+\infty} B_{k, l}=b_{k, l}, \quad B_{k, l}-b_{k, l} \varphi \in \mathcal{Y} .
\end{aligned}
$$

Moreover, we will choose the particular values

$$
\begin{aligned}
\gamma_{3,0} & :=\frac{5}{36} b_{1,0}^{4}+\frac{10}{3} d(\varepsilon) b_{1,0}+\frac{1}{2} \mu(\varepsilon) b_{1,0}^{2}, \quad \gamma_{2,1}:=\frac{1}{24} b_{1,0}^{4}-b_{1,0} b_{1,1}-4 d(\varepsilon) b_{1,0}, \\
\gamma_{1,2} & :=-\frac{3}{24} b_{1,0}^{4}+b_{1,0} b_{1,1},
\end{aligned}
$$

where $d(\varepsilon)$ satisfies (56)-(57). 
Proof. The proof of this result is easy after the validity of the following claim:

For all $(k, l) \in \Sigma_{2}$ such that $k+l=3$, we have $F_{k, l} \in \mathcal{Y}$ is odd, $G_{k, l} \in \mathcal{Y}$ is even.

Assuming (65), Lemma 2.8 is a direct consequence of Proposition 2.4

Let us prove (65). From the Appendix $\mathrm{A}$ and Proposition A.1 several (bounded but) nonlocalized terms appear in the expression of $F_{k, l}$ and $G_{k, l}$ for $k+l=3$, but all these terms eventually cancel.

Indeed, thanks to the (IP) property, terms containing derivatives of $B_{1,0}, A_{1,1}$ and $A_{2,0}$ are in $\mathcal{Y}$ as well as terms of the kind $f^{\prime}(Q) B_{1,0}$ and so on. Thus, we focus on the terms containing only $B_{1,0}, A_{1,1}$ and $A_{2,0}$ without derivatives nor multiplication by functions of $Q$. Note also that $A_{1,0} \in \mathcal{Y}$, so we also discard it. For simplicity of notation, we will skip the variables $y_{c}$ and $y$.

Now, we recollect all the non-localized terms (due to $B_{1,0}, A_{1,1}$ and $A_{2,0}$ ) in $S[\tilde{u}]$ of order $c^{l} Q_{c}^{k}$ or $c^{l}\left(Q_{c}^{k}\right)^{\prime}$ with $k+l=3$. We have only three cases: the pairs $(3,0),(2,1)$ and $(1,2)$. From Proposition A.1 we obtain

(1) (Case $(3,0))$. Here

$$
F_{3,0}=\widetilde{F}_{3,0}, \quad G_{3,0}=\widetilde{G}_{3,0}-\frac{2}{3}\left(B_{1,0}^{2}+2 A_{2,0}\right), \text { with } \widetilde{F}_{3,0}, \widetilde{G}_{3,0} \in \mathcal{Y} ;
$$

(2) (Case $(2,1))$. Here

$$
F_{2,1}=\widetilde{F}_{2,1}, \quad G_{2,1}=\widetilde{G}_{2,1}+\left(B_{1,0}^{2}+A_{1,1}+3 A_{2,0}\right) \text {, with } \widetilde{F}_{2,1}, \widetilde{G}_{2,1} \in \mathcal{Y} ;
$$

(3) (Case $(1,2)$ ). Here $F_{1,2}, G_{1,2} \in \mathcal{Y}$.

Using the following relations among the limits of $A_{2,0}, A_{1,1}$ and $B_{1,0}^{2}$ at $\pm \infty$ (see Proposition 2.6 and Lemma 2.7):

$$
\lim _{ \pm \infty} A_{2,0}=-\frac{1}{2} \lim _{ \pm \infty} B_{1,0}^{2}, \quad \lim _{ \pm \infty} A_{1,1}=-\lim _{ \pm \infty} A_{2,0},
$$

we observe that the source functions in $\left(\Omega_{k, l}\right)$ are in fact all localized. This proves (65).

2.2.6. Resolution of high order systems, cubic case. Finally we claim the existence of bounded solutions for the third and fourth order systems in the cubic case. The proof of these results is identical to the previous Lemma.

Lemma 2.9 (Resolution of $\left(\Omega_{3,0}\right),\left(\Omega_{4,0}\right)$ and $\left(\Omega_{2,1}\right)$ for $\left.m=3\right)$. For all $(k, l) \in \Sigma_{3}$ with $k \geq 2$ there exists a solution $\left(A_{k, l}, B_{k, l}, a_{k, l}\right)$ of $\left(\Omega_{k, l}\right)$ such that $A_{k, l}$ is even, $B_{k, l}$ is odd and

$$
\begin{gathered}
\lim _{+\infty} A_{k, l}=\gamma_{k, l}, \quad A_{k, l}-\gamma_{k, l} \in \mathcal{Y}, \\
\lim _{+\infty} B_{k, l}=b_{k, l}, \quad B_{k, l}-b_{k, l} \varphi \in \mathcal{Y} .
\end{gathered}
$$

In particular, we choose

$$
\gamma_{3,0}:=-\frac{1}{2} b_{1,0}^{2}, \gamma_{2,1}:=-4 b_{1,0} d(\varepsilon), \gamma_{4,0}:=3 d(\varepsilon) b_{1,0}+\frac{1}{2} \varepsilon b_{1,0}^{2} \delta_{p 4} .
$$

In this case $d(\varepsilon):=b_{2,0}(\varepsilon)$ (cf. 577)).

Proof. We note that, thanks to the (IP) property and Proposition A.1 the only a priori non localized source term is

$$
G_{2,1}=3 A_{2,0}+\tilde{G}_{2,0}, \text { with } \tilde{G}_{2,0} \in \mathcal{Y} .
$$

Then the conclusion of the Lemma follows from the fact that, from Proposition 2.6, in the cubic case, we have a priori chosen $A_{2,0} \in \mathcal{Y}$.

For further purposes, we recall the important quantities (see (29) and (56)-(57))

$$
T_{c}=c^{-\frac{1}{2}-\frac{1}{100}}, \quad d(\varepsilon)=b_{2,0}(\varepsilon)+\frac{1}{6} b_{1,0}^{3}(\varepsilon) \delta_{m 2},
$$

with $\delta_{m 2}=0$ for $m=3$, and $\delta_{22}=1$. 
2.3. Recomposition of the approximate solution. Proof of Proposition 2.1. Having solved several linear systems we now are able to prove Proposition 2.1. Indeed, we have now the enough knowlegde about the notation, so we can go further and claim the following improved result on $\tilde{u}$.

Proposition 2.10 (Construction of a symmetric approximate solution of gKdV, improved version). The solution $\tilde{u}$ above constructed satisfies, for any $0<c<c_{0}$, the following properties:

(1) For all $(t, x) \tilde{u}(t, x)=\tilde{u}(-t,-x)$.

(2) For every time $t \in\left[-T_{c}, T_{c}\right]$,

$$
\|S[\tilde{u}](t)\|_{H^{2}(\mathbb{R})} \leq K c^{\frac{3}{m-1}+\frac{3}{4}} .
$$

(3) Closeness to the sum of two soliton solution: For all time $t \in\left[-T_{c}, T_{c}\right]$, the function $\tilde{u}$ is in $H^{1}(\mathbb{R})$ and satisfies the estimate

$$
\left\|\tilde{u}(t)-Q(y)-Q_{c}\left(y_{c}\right)\right\|_{H^{1}(\mathbb{R})} \leq K_{0} c^{\frac{1}{m-1}} .
$$

(4) Closeness to a shifted two soliton solution plus a strange term: Denote

$$
\Delta_{1}:=\sum_{(k, l) \in \Sigma_{m}} a_{k, l} c^{l} \int_{\mathbb{R}} Q_{c}^{k}, \quad \tilde{b}_{1,1}:=b_{1,1}-\frac{1}{6} b_{1,0}^{3}, \quad \Delta_{2}:=2\left(b_{1,0}+c \tilde{b}_{1,1} \delta_{m 2}\right) .
$$

Then $\tilde{u}$ satisfies at time $\pm T_{c}$

$$
\begin{aligned}
& \| \tilde{u}\left( \pm T_{c}\right)-Q\left(\cdot \mp \frac{1}{2} \Delta_{1}\right)-Q_{c}\left(\cdot \pm(1-c) T_{c} \mp \frac{1}{2} \Delta_{2}\right) \\
& \left.\quad \pm d(\varepsilon)\left(Q_{c}^{2}\right)^{\prime}\left(\cdot \pm(1-c) T_{c} \mp \frac{1}{2} \Delta_{2}\right)\right\} \|_{H^{1}(\mathbb{R})} \leq K c^{\frac{3}{m-1}+\frac{1}{4}},
\end{aligned}
$$

provided for each $(k, l) \in \Sigma_{m}$, the constants $\gamma_{k, l}$ must be chosen as in Lemma [2.5, Proposition 2.6, Lemmas 2.7, 2.8 and 2.9. Recall that $d(\varepsilon)$ satisfies (56)-(57).

Remark 2.9. The quantity $\bar{b}_{1,1}$ in (70) represents the difference between the expected value of $b_{1,1}$ given by the integrable case and the actual one; namely, for $\varepsilon=0$ we have $\bar{b}_{1,1}=0$.

Proof. Let us start by proving (68). This follows from from Proposition A.1 and the choice of $a_{k, l}, A_{k, l}, B_{k, l}$ for $(k, l) \in \Sigma_{m}$, solving each linear system $\left(\Omega_{k, l}\right)$, so that

$$
S[\tilde{u}]=\mathcal{E}(t, x) .
$$

Now we deal with (69). This is an easy consequence of the fact that $y=x-\alpha, y_{c}=x+(1-c) t$, and

$$
\tilde{u}(t)-Q(y)-Q_{c}\left(y_{c}\right)=W(t, x), \quad\|W(t)\|_{H^{1}(\mathbb{R})} \leq K_{0} c^{\frac{1}{m-1}} .
$$

Proof of (71). We begin with some preliminary estimates.

Claim 4.

$$
\|\alpha\|_{L^{\infty}} \leq K c^{\frac{1}{m-1}-\frac{1}{2}}, \quad\left\|\alpha^{\prime}\right\|_{L^{\infty}} \leq K c^{\frac{1}{m-1}} .
$$

Suppose $f=f(y) \in \mathcal{Y}$. Then for all $t \in\left[-T_{c}, T_{c}\right]$,

$$
\left\|f(y) Q_{c}^{k}\left(y_{v}\right)\right\|_{L^{2}(\mathbb{R})}+\frac{1}{\sqrt{c}}\left\|f(y)\left(Q_{c}^{k}\right)^{\prime}\left(y_{v}\right)\right\|_{L^{2}(\mathbb{R})} \leq K c^{\frac{k}{m-1}} e^{-(1-c) \sqrt{c}|t|},
$$

and for $g=g(y) \in L^{\infty}(\mathbb{R})$,

$$
\left\|g(y) Q_{c}^{k}\left(y_{v}\right)\right\|_{L^{2}(\mathbb{R})}+\frac{1}{\sqrt{c}}\left\|g(y)\left(Q_{c}^{k}\right)^{\prime}\left(y_{v}\right)\right\|_{L^{2}(\mathbb{R})} \leq K c^{\frac{k}{m-1}-\frac{1}{4}} .
$$

In particular, if $t=T_{c}$ and $f \in \mathcal{Y}$, we have, for $c>0$ small,

$$
\begin{gathered}
\left\|f(y) Q_{c}\left(y_{c}\right)\right\|_{H^{1}(\mathbb{R})} \leq K c^{10} \\
\left\|Q(y)-Q\left(x-\frac{1}{2} \Delta_{1}\right)\right\|_{H^{1}(\mathbb{R})} \leq K c^{10} .
\end{gathered}
$$


Proof. The proof of these estimates are similar to Claim C.1 in the Appendix C of [26]. See also Claim 2.6 in 24. In particular for the proof we use Lemma A.2 from Appendix A] We skip the details.

We continue the proof of (71).

For the sake of brevity, we will prove only the case $m=3$. The case $m=2$ is identical to Lemma 2.6 in [26].

Note that from Claim 4

$$
\left\|Q_{c}\left(y_{c}-b_{1,0}\right)-Q_{c}+b_{1,0} Q_{c}^{\prime}-\frac{1}{2} b_{1,0}^{2} Q_{c}^{\prime \prime}\right\|_{H^{1}(\mathbb{R})} \leq K c^{\frac{7}{4}},
$$

and

$$
\left\|\left(Q_{c}^{2}\right)^{\prime}\left(y_{c}-b_{1,0}\right)-\left(Q_{c}^{2}\right)^{\prime}+b_{1,0}\left(Q_{c}^{2}\right)^{\prime \prime}\right\|_{H^{1}(\mathbb{R})} \leq K c^{\frac{9}{4}},
$$

(here we have used the fact $\left\|Q_{c}^{(3)}\right\|_{H^{1}(\mathbb{R})} \leq K c^{\frac{7}{4}}$ and $\left.\left\|\left(Q_{c}^{2}\right)^{(3)}\right\|_{H^{1}(\mathbb{R})} \leq K c^{\frac{9}{4}}\right)$. From the identities

$$
Q_{c}^{\prime \prime}=c Q_{c}-Q_{c}^{3}-\varepsilon Q_{c}^{p}+O\left(Q_{c}^{p+1}\right),\left(Q_{c}^{2}\right)^{\prime \prime}=4 c Q_{c}^{2}-3 Q_{c}^{4}+O\left(Q_{c}^{5}\right),
$$

we obtain

$$
\begin{aligned}
\| Q_{c}\left(y_{c}-b_{1,0}\right)-d(\varepsilon)\left(Q_{c}^{2}\right)^{\prime}\left(y_{c}-b_{1,0}\right) \\
\quad-\left[Q_{c}-b_{1,0} Q_{c}^{\prime}+\frac{1}{2} b_{1,0}^{2} c Q_{c}-\frac{1}{2} b_{1,0}^{2} Q_{c}^{3}-\frac{1}{2} \varepsilon b_{1,0}^{2} \delta_{p 4} Q_{c}^{4}\right] \\
\quad+d(\varepsilon)\left[\left(Q_{c}^{2}\right)^{\prime}-4 b_{1,0} c Q_{c}^{2}+3 b_{1,0} Q_{c}^{4}\right] \|_{H^{1}(\mathbb{R})} \leq K c^{\frac{7}{4}} .
\end{aligned}
$$

On the other hand, using the fact that $\lim _{+\infty} A_{k, l}=\gamma_{k, l}, \lim _{+\infty} B_{k, l}=b_{k, l}$, and Claim 4 we get

$$
\begin{gathered}
\| \tilde{u}\left(T_{c}\right)-Q-Q_{c}-b_{1,0} Q_{c}^{\prime}-\gamma_{2,0} Q_{c}^{2}-b_{2,0}\left(Q_{c}^{2}\right)^{\prime}-\gamma_{1,1} c Q_{c} \\
\quad-\gamma_{2,1} c Q_{c}^{2}-\gamma_{3,0} Q_{c}^{3}-\gamma_{4,0} Q_{c}^{4} \|_{H^{1}(\mathbb{R})} \leq K c^{7 / 4} .
\end{gathered}
$$

Combining this estimate and (79), we find

$$
\begin{aligned}
& \| \tilde{u}\left(T_{c}\right)-\left\{Q(y)+Q_{c}\left(y_{c}-b_{1,0}\right)-d(\varepsilon)\left(Q_{c}^{2}\right)^{\prime}\left(y_{c}-b_{1,0}\right)\right\} \\
& +\left(\gamma_{1,1}-\frac{1}{2} b_{1,0}^{2}\right) c Q_{c}+\gamma_{2,0} Q_{c}^{2}+\left(b_{2,0}-d(\varepsilon)\right)\left(Q_{c}^{2}\right)^{\prime}+\left(\gamma_{2,1}+4 d(\varepsilon) b_{1,0}\right) c Q_{c}^{2} \\
& +\left(\gamma_{3,0}+\frac{1}{2} b_{1,0}^{2}\right) Q_{c}^{3}+\left(\gamma_{4,0}-3 d(\varepsilon) b_{1,0}-\frac{1}{2} \varepsilon b_{1,0}^{2} \delta_{p 4}\right) Q_{c}^{4} \|_{H^{1}(\mathbb{R})} \leq K c^{\frac{7}{4}} .
\end{aligned}
$$

It follows that with the choice

we obtain

$$
\begin{aligned}
\gamma_{1,1} & =\frac{1}{2} b_{1,0}^{2}, \quad \gamma_{2,0}=0, \quad \gamma_{3,0}=-\frac{1}{2} b_{1,0}^{2}, \quad b_{2,0}=d(\varepsilon), \\
\gamma_{2,1} & =-4 d(\varepsilon) b_{1,0} \quad \text { and } \quad \gamma_{4,0}=3 d(\varepsilon) b_{1,0}+\frac{1}{2} \varepsilon b_{1,0}^{2} \delta_{p 4} .
\end{aligned}
$$

$$
\left\|\tilde{u}\left(T_{c}\right)-Q(y)-Q_{c}\left(y_{c}-b_{1,0}\right)+d(\varepsilon)\left(Q_{c}^{2}\right)^{\prime}\left(y_{c}-b_{1,0}\right)\right\|_{H^{1}(\mathbb{R})} \leq K c^{\frac{7}{4}} .
$$

The case $t=-T_{c}$ is similar and we left the proof to the reader.

Together with (76), we complete the proof of (71). This justifies in particular the choices of $\gamma_{k, l},(k, l) \in \Sigma_{m}$ done in preceding Lemmas.

2.4. Existence of the approximate pure 2-soliton collision solution. The fact that $d(\varepsilon) \neq 0$ (see Proposition 2.6) in Proposition 2.10 means formally that the collision is not elastic and that the residue due to the collision is of order $\left(Q_{c}^{2}\right)^{\prime}$. However, the approximate solution $\tilde{u}(t, x)$ given in Lemma 2.10 being symmetric, it contains the residue at both $-T_{c}$ and $T_{c}$ (see (171)). To match the solution $u(t)$ considered in Theorem 1.4 which is pure at $-\infty$, we need to introduce a modified approximate solution, which, at main order, will contain a residue only at time $t=T_{c}$. This will be clear after the following

Proposition 2.11. There exists a function $\hat{u}=\hat{u}(t, x)$, of the form given by (33) such that for some constants $K, c_{0}>0$ and $0<c<c_{0}$, the following estimates hold: 
(1) $\hat{u}(t, x) \not \equiv \hat{u}(-t,-x)$ for every $t, x$.

(2) Almost solution. For any $t \in\left[-T_{c}, T_{c}\right]$,

$$
\|S[\hat{u}](t)\|_{H^{1}(\mathbb{R})} \leq K c^{\frac{2}{m-1}+\frac{3}{4}}\left[c^{\frac{1}{m-1}}+|d(\varepsilon)| c^{\frac{1}{4}}\right],
$$

(recall that $d(\varepsilon)$ measures the residue after the collision, introduced in (56)-(57)).

(3) Closeness to a two-soliton solution at time $t=-T_{c}$. With the defintions of shifts given in (70), the modified function $\hat{u}$ is close to a two solitons solution at time $-T_{c}$ :

$$
\left\|\hat{u}\left(-T_{c}\right)-\left\{Q\left(\cdot+\frac{1}{2} \Delta_{2}\right)+Q_{c}\left(\cdot+(1-c) T_{c}+\frac{1}{2} \Delta_{2}\right)\right\}\right\|_{H^{1}(\mathbb{R})} \leq K c^{\frac{2}{m-1}+\frac{1}{4}}\left[c^{\frac{1}{m-1}}+|d(\varepsilon)| c^{\frac{1}{2}}\right] .
$$

(4) Non-matching with a two-soliton solution at time $t=T_{c}$ :

$$
\begin{aligned}
& \| \hat{u}\left(T_{c}\right)-Q\left(\cdot-\frac{1}{2} \Delta_{1}\right)-Q_{c}\left(\cdot+(1-c) T_{c}-\frac{1}{2} \Delta_{2}\right) \\
& \quad+2 d(\varepsilon)\left(Q_{c}^{2}\right)^{\prime}\left(\cdot+(1-c) T_{c}-\frac{1}{2} \Delta_{2}\right) \|_{H^{1}(\mathbb{R})} \leq K c^{\frac{2}{m-1}+\frac{1}{4}}\left[c^{\frac{1}{m-1}}+|d(\varepsilon)| c^{\frac{1}{2}}\right] .
\end{aligned}
$$

where, from (56)-(57),

$$
\forall 0<|\varepsilon| \leq \varepsilon_{0}, \quad d(\varepsilon)=c_{m, p} \varepsilon+o(\varepsilon),
$$

and

$$
\left|\Delta_{1}-a_{1,0} \int_{\mathbb{R}} Q_{c}\right| \leq K c^{\frac{2}{m-1}-\frac{1}{2}}, \quad\left|\Delta_{2}-2 b_{1,0}\right| \leq K c .
$$

(5) Comparison residue versus error terms: The residue in (83) satisfies

$$
\begin{aligned}
\left\|2 d(\varepsilon)\left(Q_{c}^{2}\right)^{\prime}\left(\cdot+(1-c) T_{c}-\frac{1}{2} \Delta_{2}\right)\right\|_{H^{1}(\mathbb{R})} & \sim|d(\varepsilon)| c^{\frac{2}{m-1}+\frac{1}{4}} \\
& \gg c^{\frac{2}{m-1}+\frac{1}{4}}\left[c^{\frac{1}{m-1}}+|d(\varepsilon)| c^{\frac{1}{2}}\right],
\end{aligned}
$$

provided $c^{\frac{1}{m-1}} \ll|d(\varepsilon)|$.

Remark 2.10. The approximate solution $\hat{u}$ above mentioned describes the collision of two pure solitons that at time $t \sim T_{c}$ (after colliding) differ by a term of order $|d(\varepsilon)| c^{\frac{2}{m-1}+\frac{1}{4}}$ of the ingoing solitons before the collision, at time $t \sim-T_{c}$, provided (23) holds.

For even small values of $\varepsilon$ such that condition (23) does not hold, we need to go further in our approximate solution and solve even more linear systems. We believe that in this case, more involved, the conclusions of this paper are the same.

Let us return to the proof of Proposition 2.11.

Proof. Let $\hat{u}:=\tilde{u}+w_{\#}$, where

$$
w_{\#}(t, x):=-d(\varepsilon)\left(Q_{c}^{2}\right)^{\prime}\left(y_{c}\right)(1+\bar{P}(y)),
$$

and $\bar{P}$ was defined in (39). Now $w_{\#}$ can be expressed in the form

$$
w_{\#}(t, x)=Q(y)+Q_{c}\left(y_{c}\right)+\sum_{(k, l) \in \Sigma_{m}} c^{l}\left\{\hat{A}_{k, l}(y) Q_{c}^{k}\left(y_{c}\right)+\hat{B}_{k, l}(y)\left(Q_{c}^{k}\right)^{\prime}\left(y_{c}\right)\right\},
$$

where $\hat{A}_{k, l}=A_{k, l}, \hat{B}_{k, l}=B_{k, l}+w_{\#} \delta_{(k, l),(2,0)}$. Here $\delta_{(2,0),(2,0)}=1$ and $\delta_{(k, l),(2,0)}=0$ otherwise.

Let us prove (83). Replacing $\tilde{u}=\hat{u}-w_{\#}$ in (71), we have

$$
\begin{aligned}
& \| \hat{u}\left(T_{c}\right)-Q\left(\cdot-\frac{1}{2} \Delta_{1}\right)-Q_{c}\left(\cdot+(1-c) T_{c}-\frac{1}{2} \Delta_{2}\right) \\
& \quad+d(\varepsilon)\left(Q_{c}^{2}\right)^{\prime}\left(\cdot+(1-c) T_{c}-\frac{1}{2} \Delta_{2}\right)-w_{\#}\left(T_{c}\right) \|_{H^{1}(\mathbb{R})} \leq K c^{\frac{3}{m-1}+\frac{1}{4}} .
\end{aligned}
$$


Thus, using (75) (note that $\bar{P} \in \mathcal{Y}$ )

$$
\begin{aligned}
& \left\|\hat{u}\left(T_{c}\right)-Q\left(\cdot-\frac{1}{2} \Delta_{1}\right)-Q_{c}\left(\cdot+(1-c) T_{c}-\frac{1}{2} \Delta_{2}\right)+2 d(\varepsilon)\left(Q_{c}^{2}\right)^{\prime}\left(\cdot+(1-c) T_{c}-\frac{1}{2} \Delta_{2}\right)\right\|_{H^{1}(\mathbb{R})} \\
& \leq K c^{\frac{3}{m-1}+\frac{1}{4}}+\left\|d(\varepsilon)\left(Q_{c}^{2}\right)^{\prime}\left(\cdot+(1-c) T_{c}-\frac{1}{2} \Delta_{2}\right)+w_{\#}\left(T_{c}\right)\right\|_{H^{1}(\mathbb{R})} \\
& \leq K c^{\frac{3}{m-1}+\frac{1}{4}}+K|d(\varepsilon)|\left\|\left(Q_{c}^{2}\right)^{\prime}\left(\cdot-\frac{1}{2} \Delta_{2}\right)-\left(Q_{c}^{2}\right)^{\prime}\right\|_{H^{1}(\mathbb{R})} \\
& \leq K c^{\frac{3}{m-1}+\frac{1}{4}}+K|d(\varepsilon)| c^{\frac{2}{m-1}+\frac{3}{4}} .
\end{aligned}
$$

Similarly, at time $t=-T_{c}$

$$
\begin{aligned}
& \| \hat{u}\left(-T_{c}\right)-Q\left(\cdot+\frac{1}{2} \Delta_{1}\right)-Q_{c}\left(\cdot-(1-c) T_{c}+\frac{1}{2} \Delta_{2}\right) \\
& \quad-d(\varepsilon)\left(Q_{c}^{2}\right)^{\prime}\left(\cdot-(1-c) T_{c}+\frac{1}{2} \Delta_{2}\right)-w_{\#}\left(-T_{c}\right) \|_{H^{1}(\mathbb{R})} \leq K c^{\frac{3}{m-1}+\frac{1}{4}}
\end{aligned}
$$

so that

$$
\begin{aligned}
\| \hat{u}\left(-T_{c}\right) & -Q\left(\cdot+\frac{1}{2} \Delta_{1}\right)-Q_{c}\left(\cdot-(1-c) T_{c}+\frac{1}{2} \Delta_{2}\right) \|_{H^{1}(\mathbb{R})} \\
\leq & K c^{\frac{3}{m-1}+\frac{1}{4}}+K|d(\varepsilon)|\left\|\left(Q_{c}^{2}\right)^{\prime}\left(\cdot+\frac{1}{2} \Delta_{2}\right)-\left(Q_{c}^{2}\right)^{\prime}\right\|_{H^{1}(\mathbb{R})} \\
\leq & K c^{\frac{3}{m-1}+\frac{1}{4}}+K|d(\varepsilon)| c^{\frac{2}{m-1}+\frac{3}{4}} .
\end{aligned}
$$

Note that (84) is clearly a consequence of (70).

Finally, we prove (81). Note that (cf. Appendix B for the definitions)

$$
\begin{aligned}
S[\hat{u}] & =S\left[\tilde{u}+w_{\#}\right] \\
& =S[\tilde{u}]+\mathbf{I I I}\left(w_{\#}\right)+\left[f\left(\tilde{u}+w_{\#}\right)-f(\tilde{u})-f^{\prime}(Q) w_{\#}\right]_{x}
\end{aligned}
$$

The following estimates allow to conclude (81). We claim

Claim 5. With the choice of $w_{\#}$ given in (85),

$$
\left\|\mathbf{I I I}\left(w_{\#}\right)\right\|_{H^{1}(\mathbb{R})} \leq K|d(\varepsilon)| c^{1+\frac{2}{m-1}} .
$$

and

$$
\left\|\left[f\left(\tilde{u}+w_{\#}\right)-f(\tilde{u})-f^{\prime}(Q) w_{\#}\right]_{x}\right\|_{H^{1}(\mathbb{R})} \leq K|d(\varepsilon)| c^{\frac{1}{2}+\frac{3}{m-1}}
$$

Proof. The proof is similar to the proof of Proposition A.1 above. We only sketch the main ideas.

Let us prove (86). First, note that for $\bar{P}$ defined in (39)

$$
(\mathcal{L}(1+\bar{P}))^{\prime}=\left(1-f^{\prime}(Q)+f^{\prime}(Q)\right)^{\prime}=0 .
$$

This property will be useful in what follows. From the calculations performed in (138), (88) and the fact that $(1+\bar{P})^{\prime} \in \mathcal{Y}$, we note that (cf. (131) and (137) for the definition of $\mathbf{I I I}(\cdot)$ and $\Sigma_{m}^{\prime}$ respectively)

$$
\begin{aligned}
\operatorname{III}\left((1+\bar{P})\left(Q_{c}^{2}\right)^{\prime}\right)= & -(\mathcal{L}(1+\bar{P}))^{\prime}\left(Q_{c}^{2}\right)^{\prime}-c(1+\bar{P})\left(Q_{c}^{2}\right)^{\prime \prime}+(1+\bar{P})\left(Q_{c}^{2}\right)^{(3)} \\
& +\sum_{(k, l) \in \Sigma_{m}^{\prime}} c^{l}\left[\tilde{F}_{k, l} Q_{c}^{k}+\tilde{G}_{k, l}\left(Q_{c}^{k}\right)^{\prime}\right]+O\left(c Q_{c}^{3}+Q_{c}^{5}+c^{2} Q_{c}\right) \\
= & \sum_{(k, l) \in \Sigma_{m}^{\prime}} c^{l}\left[\tilde{F}_{k, l} Q_{c}^{k}+\tilde{G}_{k, l}\left(Q_{c}^{k}\right)^{\prime}\right]+O\left(c\left(Q_{c}^{2}\right)^{\prime}+c Q_{c}^{3}+Q_{c}^{5}+c^{2} Q_{c}\right),
\end{aligned}
$$

where both $\tilde{F}_{k, l}$ and $\tilde{G}_{k, l}$ are in $\mathcal{Y}$. Moreover, $\tilde{F}_{3,0}=0$. From here, the definition of $w_{\#}$ in (85) and Claim 4 , we obtain

$$
\left\|\mathbf{I I I}\left(w_{\#}\right)\right\|_{H^{2}(\mathbb{R})} \leq K|d(\varepsilon)| c^{\frac{2}{m-1}+1} .
$$

Now, we deal with (87). We note that

$$
\begin{aligned}
\text { (87) }= & {\left[f\left(\tilde{u}+w_{\#}\right)-f(\tilde{u})-f^{\prime}(\tilde{u}) w_{\#}\right]_{x}+\left[\left(f^{\prime}(\tilde{u})-f^{\prime}(Q)\right) w_{\#}\right]_{x} } \\
& {\left[\frac{1}{2} f^{\prime \prime}(\tilde{u}) w_{\#}^{2}+O\left(w_{\#}^{3}\right)\right]_{x}+\left[f^{\prime \prime}(Q)(\tilde{u}-Q) w_{\#}+\frac{1}{2} f^{(3)}(Q)(\tilde{u}-Q)^{2} w_{\#}+O\left((\tilde{u}-Q)^{3} w_{\#}\right)\right]_{x} . }
\end{aligned}
$$


From here, using the expresion for $w_{\#}$ and Claim 4, we obtain

$$
\|\left(\text { [87) } \|_{H^{2}} \leq K|d(\varepsilon)|\left[|d(\varepsilon)| c^{\frac{3}{2}+\frac{4}{m-1}}+c^{\frac{1}{2}+\frac{4}{m-1}}+c^{\frac{1}{2}+\frac{3}{m-1}}\right] \leq K|d(\varepsilon)| c^{\frac{1}{2}+\frac{3}{m-1}} .\right.
$$

This finishes the proof.

This Claim allows us to finish the proof of the Proposition.

\section{Preliminary Results for stability of the 2-Soliton structure}

In this section several stability results will allow to study the long time behavior of the 2-soliton soliton solution. First of all, we recall a general result proved in 25] concerning the existence and properties of an actual function $u=u(t, x)$, solution of (28) in the interval $\left[-T_{c}, T_{c}\right]$ and close enough to our approximate solution $\hat{u}$. This will be done in the next subsection.

Next, we study the stability of a solution $u(t)$ of (21) for long time, namely $t \geq T_{c}$. These results have been proved in great generality by Martel and Merle in [22, [25], and [23. In particular, we will use the stability and asymptotic stability of the two solitons (Proposition 3.3) to show the persistence of the 2-soliton structure for long time.

Finally, a key result is the decomposition result from Lemma 3.4, which will be essential to show the persistence of the residual term (cf. (83)) at infinity.

3.1. Dynamic stability in the interaction region. For any $c>0$ sufficiently small, we will consider the function $\hat{u}(t)$ of the form

$$
\hat{u}(t, x)=Q(y)+Q_{c}\left(y_{c}\right)+\sum_{(k, l) \in \Sigma_{m}} c^{l}\left\{Q_{c}^{k}\left(y_{c}\right) \hat{A}_{k, l}(y)+\left(Q_{c}^{k}\right)^{\prime}\left(y_{c}\right) \hat{B}_{k, l}(y)\right\}
$$

defined in Proposition 2.11 (the notation was introduced in (32) and (33)). Recall the error term

$$
S[\hat{u}](t)=\hat{u}_{t}+\left(\hat{u}_{x x}-u+f(\hat{u})\right)_{x} .
$$

Proposition 3.1 (Exact solution close to the approximate solution $\hat{u},[25]$ ). Let $\theta>\frac{1}{m-1}$ and $\varepsilon$ small enough such that (12) holds for $Q$. There exists $c_{0}>0$ such that the following holds for any $0<c<c_{0}$. Suppose that for all $t \in\left[-T_{c}, T_{c}\right]$

$$
\|S[\hat{u}](t)\|_{H^{2}(\mathbb{R})} \leq K \frac{c^{\theta}}{T_{c}}
$$

and for some $T_{0} \in\left[-T_{c}, T_{c}\right]$,

$$
\left\|u\left(T_{0}\right)-\hat{u}\left(T_{0}\right)\right\|_{H^{1}(\mathbb{R})} \leq K c^{\theta},
$$

where $u(t)$ is an $H^{1}$ solution of (28). Then, there exist $K_{0}=K_{0}(\theta, K, f)$ and a $C^{1}$ function $\rho:\left[-T_{c}, T_{c}\right] \rightarrow \mathbb{R}$ such that, for all $t \in\left[-T_{c}, T_{c}\right]$,

$$
\|u(t)-\hat{u}(t, \cdot-\rho(t))\|_{H^{1}(\mathbb{R})} \leq K_{0} c^{\theta}, \quad\left|\rho^{\prime}(t)-1\right| \leq K_{0} c^{\theta} .
$$

Remark 3.1. The proof of the above Proposition is nontrivial and requires some refined techniques such as modulation theory, coercivity properties and the introduction of a modified energy functional adapted to a two soliton collision. It is necessary to emphasize that one of the key elements in the proof is the smallness of the error term $S[\hat{u}]$ along the collision. For the sake of completeness, we will draw the main lines of the argument, see 25] for the actual complete proof.

Proof. It suffices to show the result on the interval $\left[T_{0}, T_{c}\right]$. By using the transformation $x \rightarrow-x$, $t \rightarrow-t$, the proof is the same on $\left[-T_{c}, T_{0}\right]$.

Let $K^{*}>1$ be a constant to be fixed later. Since $\left\|u\left(T_{0}\right)-\hat{u}\left(T_{0}\right)\right\|_{H^{1}(\mathbb{R})} \leq c^{\theta}$, by continuity in time in $H^{1}(\mathbb{R})$, there exists $T_{0}<T^{*} \leq T_{c}$ such that

$$
\begin{gathered}
T^{*}=\sup \left\{T \in\left[T_{0}, T_{c}\right] \text { such that for all } t \in\left[T_{0}, T\right], \text { there exists } r(t) \in \mathbb{R}\right. \text { with } \\
\left.\|u(t)-\hat{u}(t, \cdot-r(t))\|_{H^{1}(\mathbb{R})} \leq K^{*} c^{\theta}\right\} .
\end{gathered}
$$


The objective is to prove that $T^{*}=T_{c}$ for $K^{*}$ large. For this, we argue by contradiction, assuming that $T^{*}<T_{c}$ and reaching a contradiction with the definition of $T^{*}$ by proving some independent estimates on $\|u(t)-\hat{u}(t, \cdot-r)\|_{H^{1}(\mathbb{R})}$ on $\left[T_{0}, T^{*}\right]$.

An argument using the Implicit function theorem allows to construct a modulation parameter and to estimate its variation in time:

Claim 6. Assume that $0<c<c\left(K^{*}\right)$ small enough. There exists a unique $C^{1}$ function $\rho(t)$ such that, for all $t \in\left[T_{0}, T^{*}\right]$,

$$
z(t, x)=u(t, x+\rho(t))-\hat{u}(t, x) \quad \text { satisfies } \quad \int_{\mathbb{R}} z(t, x) Q^{\prime}(y) d x=0 .
$$

Moreover, we have, for all $t \in\left[T_{0}, T^{*}\right]$,

$$
\begin{gathered}
\left|\rho\left(T_{0}\right)\right|+\left\|z\left(T_{0}\right)\right\|_{H^{1}(\mathbb{R})} \leq K c^{\theta},\|z(t)\|_{H^{1}(\mathbb{R})} \leq 2 K^{*} c^{\theta} \\
z_{t}+\left(z_{x x}-z+f(z+\hat{u})-f(\hat{u})\right)_{x}=-S[\hat{u}](t)+\left(\rho^{\prime}(t)-1\right)(\hat{u}+z)_{x} \\
\left|\rho^{\prime}(t)-1\right| \leq K\|z(t)\|_{H^{1}(\mathbb{R})}+K\|S[\hat{u}](t)\|_{H^{1}(\mathbb{R})},
\end{gathered}
$$

The purpose of the modulation theory is to establish a lower bound in the following energy functional for $z(t)$ :

$$
\mathcal{F}(t):=\frac{1}{2} \int_{\mathbb{R}}\left(\left(\partial_{x} z\right)^{2}+\left(1+\alpha^{\prime}\left(y_{c}\right)\right) z^{2}\right)-\int_{\mathbb{R}}(F(\hat{u}+z)-F(\hat{u})-f(\hat{u}) z) .
$$

Indeed, this functional enjoys two useful properties: it has a very small time variation and it is coercive up to the direction $Q$ :

Lemma 3.2 (Coercivity of $\mathcal{F})$. Assume that $0<c<c\left(K^{*}\right)$ small enough. There exists $K>0$ (independent of $K^{*}$ and $c$ ) such that

(1) Coercivity of $\mathcal{F}$ under orthogonality conditions:

$$
\forall t \in\left[T_{0}, T^{*}\right], \quad\|z(t)\|_{H^{1}(\mathbb{R})}^{2} \leq K \mathcal{F}(t)+K\left|\int_{\mathbb{R}} z(t) Q(y)\right|^{2} .
$$

(2) Control of the direction $Q$ :

$$
\forall t \in\left[T_{0}, T^{*}\right], \quad\left|\int_{\mathbb{R}} z(t) Q(y)\right| \leq K c^{\theta}+K c^{\frac{1}{p-1}-\frac{1}{4}}\|z(t)\|_{L^{2}}+K\|z(t)\|_{L^{2}}^{2} .
$$

(3) Control of the variation of the energy functional:

$$
\mathcal{F}\left(T^{*}\right)-\mathcal{F}\left(T_{0}\right) \leq K c^{2 \theta}\left(\left(K^{*}\right)^{2}\left(1+K^{*}\right) c^{\frac{1}{2(m-1)}-\frac{1}{8}}+K^{*}\right)
$$

These estimates allow us, after fixing $K^{*}$ large enough and possibly taking $c$ even smaller, to show that actually

$$
\left\|z\left(T^{*}\right)\right\|_{H^{1}(\mathbb{R})}^{2} \leq \frac{1}{2}\left(K^{*}\right)^{2} c^{2 \theta} .
$$

contradicting the definition of $T^{*}$, thus proving that $T^{*}=T_{c}$.

Once the existence of an actual solution (close to our approximate solution $\hat{u}$ in the interval $\left.\left[-T_{c}, T_{c}\right]\right)$ is established, one would like to investigate the behavior in long time of this solution. We treat this problem in the next subsection. 
3.2. Stability and asymptotic stability for large time. Here we consider the stability of the 2 -soliton structure after the collision, and for a long time. Let $T_{c}$ be defined in (29). We start with an important

Remark 3.2. Since (12) holds for $f(s)=s^{m}, m=2,3$, it is clear by a perturbation argument that (12) holds also for $f$ as in (22) for all $0<c<1$, provided $0<|\varepsilon|<\varepsilon_{0}$ is small enough.

Proposition 3.3 (Stability of two decoupled solitons, 22, [23]). Let $\varepsilon$ small enough such that (12) holds for $Q$. Then there exist constants $c_{0}, K>0$, such that for any $0<c<c_{0}$ and for any $\omega>0$, the following holds. Let $u(t)$ be an $H^{1}$ solution of (28) such that for some time $t_{1} \in \mathbb{R}$ and $\frac{1}{2} T_{c} \leq X_{0} \leq \frac{3}{2} T_{c}$,

$$
\left\|u\left(t_{1}\right)-Q-Q_{c}\left(\cdot+X_{0}\right)\right\|_{H^{1}(\mathbb{R})} \leq c^{\frac{1}{4}+\frac{1}{m-1}+\omega} .
$$

Then there exist $C^{1}$-functions $\rho_{1}(t), \rho_{2}(t)$ defined on $\left[t_{1},+\infty\right)$ such that

(1) Stability:

$$
\sup _{t \geq t_{1}}\left\|u(t)-Q\left(\cdot-\rho_{1}(t)\right)-Q_{c}\left(\cdot-\rho_{2}(t)\right)\right\|_{H^{1}(\mathbb{R})} \leq K c^{-\frac{1}{4}+\frac{1}{m-1}+\omega},
$$

and for all $t \geq t_{1}$

$$
\frac{1}{2} \leq \rho_{1}^{\prime}(t)-\rho_{2}^{\prime}(t) \leq \frac{3}{2}, \quad\left|\rho_{1}\left(t_{1}\right)\right| \leq K c^{\frac{1}{4}+\frac{1}{m-1}+\omega}, \quad\left|\rho_{2}\left(t_{1}\right)+X_{0}\right| \leq K c^{\omega} .
$$

(2) Asymptotic stability: There exist $c_{1}^{+}, c_{2}^{+}>0$ such that on the right hand side limit

$$
\left.\lim _{t \rightarrow+\infty} \| u(t)-Q_{c_{1}^{+}}\left(x-\rho_{1}(t)\right)-Q_{c_{2}^{+}}\left(x-\rho_{2}(t)\right)\right) \|_{H^{1}\left(x>\frac{1}{10} c t\right)}=0,
$$

with

$$
\left|c_{1}^{+}-1\right| \leq K c^{\frac{1}{4}+\frac{1}{m-1}+\omega}, \quad\left|\frac{c_{2}^{+}}{c}-1\right| \leq K c^{\omega} .
$$

3.3. A decomposition result. Recall a more precise decomposition of $u(t)$ used in the proof of Proposition 3.3 in 22], 23].

Lemma 3.4 (Decomposition of the solution, [23]). Suppose (12) holds for Q. Let $u=u(t)$ be a solution of the $g K d V$ equation (21) such that the estimate (92) holds. Then there exist $C^{1}$-functions $\rho_{1}(t), \rho_{2}(t), c_{1}(t), c_{2}(t)$, defined on $\left[t_{1},+\infty\right)$, such that the function

$$
\eta(t, x):=u(t, x)-R_{1}(t, x)-R_{2}(t, x),
$$

where, for $j=1,2, R_{j}(t, x):=Q_{c_{j}(t)}\left(x-\rho_{j}(t)\right)$, satisfies for all $t \geq t_{1}$,

$$
\begin{aligned}
& \int_{\mathbb{R}} R_{j}(t) \eta(t)=\int_{\mathbb{R}}\left(x-\rho_{j}(t)\right) R_{j}(t) \eta(t)=0, \quad j=1,2, \\
& \|\eta(t)\|_{H^{1}(\mathbb{R})}+\left|c_{1}(t)-1\right|+c^{\frac{1}{m-1}-\frac{1}{4}}\left|\frac{c_{2}(t)}{c}-1\right| \leq K c^{\omega+\frac{1}{m-1}-\frac{1}{4}}, \\
& \quad \text { and for all } t \geq t_{1} \quad\left|\rho_{2}^{\prime}(t)\right|+\left|\rho_{1}^{\prime}(t)-1\right| \leq \frac{1}{10}, \quad \rho_{1}(t)-\rho_{2}(t) \geq \frac{1}{2} t+\frac{1}{4} T_{c} .
\end{aligned}
$$

Moreover, we have the convergence $\lim _{t \rightarrow+\infty} \bar{c}_{j}(t)=c_{j}^{+}$for $j=1,2$.

At this moment we have all the necessary information about the 2-soliton solution of (28). Indeed, recall from the sketch of proof (Subsection 1.4) that the asymptotic in long time will be treated using the tools from this section, more precisely using Proposition 3.3 and Lemma 3.4 On the other hand the collision region will be described by Proposition 3.1. This is the purpose of the next section. 


\section{Proof of the Theorem 1.4}

Now we are in a position to prove the main Theorem of this work.

Proof of Theorem 1.4. Let $1=c_{1}<c_{*}(f)$ such that (12) holds and $0<c<c_{0}(\varepsilon)$ small enough (depending on $\varepsilon$ ). Let $u(t)$ be the unique solution of (21) such that (see Theorem 1 and Remark 2 in [19])

$$
\lim _{t \rightarrow-\infty}\left\|u(t)-Q(x-t)-Q_{c}(x-c t)\right\|_{H^{1}(\mathbb{R})}=0 .
$$

1. Behavior at $-T_{c}$. We claim that for all $t<-\frac{1}{32} T_{c}$,

$$
\left\|u(t)-Q(\cdot-t)-Q_{c}(\cdot-c t)\right\|_{H^{1}(\mathbb{R})} \leq K e^{\frac{1}{4} \sqrt{c}(1-c) t} .
$$

This is a consequence of the proof of existence of $u(t)$ in [19]. See Proposition 5.1 in 24] for a proof in the power case.

Now, using (100), we will match the function $u$ with the collision solution $\hat{u}$ constructed in Proposition 2.11 For this, we will translate $u$ in time and space, as follows.

Let $\Delta_{1}, \Delta_{2}$ be defined in Proposition 2.10 and

$$
T_{c}^{-}:=T_{c}+\frac{1}{2} \frac{\Delta_{1}-\Delta_{2}}{1-c}, \quad a:=\frac{1}{2} \Delta_{1}-T_{c}^{-} .
$$

Since from (84)

$$
\left|\Delta_{1}\right| \leq K c^{\frac{1}{m-1}-\frac{1}{2}}, \quad \text { and } \quad\left|\Delta_{2}\right| \leq K
$$

we have $-T_{c}^{-} \leq-\frac{1}{32} T_{c}$, and thus, from (100) for $c$ small enough, and after a translation by $a$, we get

$$
\left\|u\left(-T_{c}^{-}, \cdot+a\right)-Q\left(\cdot+\frac{\Delta_{1}}{2}\right)-Q_{c}\left(\cdot-(1-c) T_{c}+\frac{\Delta_{2}}{2}\right)\right\|_{H^{1}(\mathbb{R})} \leq K e^{-\frac{1}{4} \sqrt{c}(1-c) T_{c}^{-}} \leq K c^{10} .
$$

By translation invariance, we may assume $T_{c}^{-}=T_{c}$ and $a=0$, such that

$$
\left\|u\left(-T_{c}\right)-Q\left(\cdot+\frac{\Delta_{1}}{2}\right)-Q_{c}\left(\cdot-(1-c) T_{c}+\frac{\Delta_{2}}{2}\right)\right\|_{H^{1}(\mathbb{R})} \leq K c^{10} .
$$

2. Behavior at $+T_{c}$. Now, possibly taking a smaller $c$, consider $\hat{u}=\hat{u}_{1, c}$ constructed in Proposition 2.11 By (82) and (101), we have

$$
\left\|u\left(-T_{c}\right)-\hat{u}\left(-T_{c}\right)\right\|_{H^{1}(\mathbb{R})} \leq K c^{\frac{2}{m-1}+\frac{1}{4}}\left[c^{\frac{1}{m-1}}+|d(\varepsilon)| c^{\frac{1}{2}}\right] .
$$

Applying Proposition 3.1 with

$$
T_{0}=-T_{c}, \quad c^{\theta}:=c^{\frac{2}{m-1}+\frac{1}{4}-\frac{1}{100}}\left[c^{\frac{1}{m-1}}+|d(\varepsilon)| c^{\frac{1}{4}}\right],
$$

it follows that there exists a function $\rho(t)$ such that for all $t \in\left[-T_{c}, T_{c}\right]$,

$$
\|u(t)-\hat{u}(t, \cdot-\rho(t))\|_{H^{1}(\mathbb{R})} \leq K c^{\theta} .
$$

In particular, for $r:=\rho\left(T_{c}\right)$, we have

$$
\left\|u\left(T_{c}\right)-\hat{u}\left(T_{c}, \cdot-r\right)\right\|_{H^{1}(\mathbb{R})} \leq K c^{\theta} .
$$

Using (83) and triangular inequality, we obtain

$$
\left\|u\left(T_{c}\right)-Q\left(\cdot-r_{1}\right)-Q_{c}\left(\cdot-r_{2}\right)-2 d(\varepsilon)\left(Q_{c}^{2}\right)^{\prime}\left(\cdot-r_{2}\right)\right\|_{H^{1}(\mathbb{R})} \leq K c^{\theta} .
$$

Here,

$$
r_{1}:=\frac{1}{2} \Delta_{1}+r, \quad r_{2}:=(c-1) T_{c}+\frac{1}{2} \Delta_{2}+r,
$$

so that $r_{1}-r_{2}=(1-c) T_{c}+\frac{1}{2}\left(\Delta_{1}-\Delta_{2}\right)$ satisfies

$$
\frac{1}{2}(1-c) T_{c} \leq r_{1}-r_{2} \leq 32(1-c) T_{c} .
$$

Moreover, note that

$$
\left\|\left(Q_{c}^{2}\right)^{\prime}\right\|_{H^{1}(\mathbb{R})} \leq K c^{\frac{2}{m-1}+\frac{1}{4}}
$$


so that

$$
\left\|u\left(T_{c}\right)-Q\left(\cdot-r_{1}\right)-Q_{c}\left(\cdot-r_{2}\right)\right\|_{H^{1}(\mathbb{R})} \leq K c^{\frac{2}{m-1}+\frac{1}{4}}\left[|d(\varepsilon)|+c^{\frac{1}{m-1}}\right] \leq K|d(\varepsilon)| c^{\frac{2}{m-1}+\frac{1}{4}},
$$

provided

$$
|d(\varepsilon)| \geq \kappa_{0} c^{\frac{1}{m-1}-\frac{1}{100}}
$$

for some $\kappa_{0}>0$ large enough but fixed. We have thus arrived to time $t=+T_{c}$ with a stability property of the 2 -soliton structure, namely (104).

3. Behavior as $t \rightarrow+\infty$. From (104), it follows that we can apply Proposition 3.3 to $u\left(t, \cdot+r_{1}\right)$ for $t \geq T_{c}$ (that is, $t_{1}:=T_{c}$ ), with $X_{0}:=r_{1}-r_{2}$, and

$$
c^{\omega}:=|d(\varepsilon)| c^{\frac{1}{m-1}} .
$$

It follows that there exist $\rho_{1}(t), \rho_{2}(t), c_{1}^{+}>0, c_{2}^{+}>0$ so that

$$
w^{+}(t, x):=u(t, x)-Q_{c_{1}^{+}}\left(x-r_{1}-\rho_{1}(t)\right)-Q_{c_{2}^{+}}\left(x-r_{1}-\rho_{2}(t)\right)
$$

satisfies

$$
\sup _{t \geq T_{c}}\left\|w^{+}(t)\right\|_{H^{1}(\mathbb{R})} \leq K|d(\varepsilon)| c^{\frac{2}{m-1}-\frac{1}{4}}, \quad \lim _{t \rightarrow+\infty}\left\|w^{+}(t)\right\|_{H^{1}\left(x>\frac{c}{10} t+r_{1}\right)}=0
$$

and

$$
\left|c_{1}^{+}-1\right| \leq K|d(\varepsilon)| c^{\frac{2}{m-1}+\frac{1}{4}}, \quad\left|c_{2}^{+}-c\right| \leq K|d(\varepsilon)| c^{1+\frac{1}{m-1}} .
$$

In particular, the behavior of the 2 -soliton structure remains stable at infinity, modulo the emergency of a possible $H^{1}$-nonzero residual term. This proves the upper bound in (26). At this stage, we do not know if this residual term (that is, $w^{+}$) can be bounded by below uniformly in time. This is the purpose of the following key step.

4. Lower bound on $w^{+}(t)$ for $t>T_{c}$ large. Consider the decomposition of $u\left(\cdot, \cdot+r_{1}\right)$ defined in Lemma 3.4, i.e. the center of mass $\bar{\rho}_{1}(t), \bar{\rho}_{2}(t)$, the scaling parameters $\bar{c}_{1}(t), \bar{c}_{2}(t)$ such that, for $t>T_{c}$,

satisfies

$$
\eta(t, x):=u(t, x)-Q_{\bar{c}_{1}(t)}\left(x-r_{1}-\bar{\rho}_{1}(t)\right)-Q_{\bar{c}_{2}(t)}\left(x-r_{1}-\bar{\rho}_{2}(t)\right)
$$

$$
\begin{aligned}
& \sup _{t \geq T_{c}}\|\eta(t)\|_{H^{1}(\mathbb{R})} \leq K|d(\varepsilon)| c^{\frac{2}{m-1}-\frac{1}{4}}, \quad\left|\bar{c}_{1}\left(T_{c}\right)-1\right| \leq K|d(\varepsilon)| c^{\frac{2}{m-1}-\frac{1}{4}}, \\
& \bar{\rho}_{1}(t)-\bar{\rho}_{2}(t) \geq \frac{1}{2} t+\frac{1}{4} T_{c}, \quad\left|\bar{c}_{2}(t)-c\right| \leq K|d(\varepsilon)| c^{\frac{1}{m-1}+\frac{3}{2}}
\end{aligned}
$$

and

$$
\left|\bar{\rho}_{2}\left(T_{c}\right)+r_{1}-r_{2}\right| \leq K|d(\varepsilon)| c^{\frac{1}{m-1}} .
$$

Moreover, we have for $j=1,2$

$$
\lim _{t \rightarrow+\infty} \bar{c}_{j}(t)=c_{j}^{+} .
$$

First, as a consequence of (102), we claim the following lower bound at $t=T_{c}$ : for $K_{0}>0$, independent of $c>0$,

$$
\int_{x<\bar{\rho}_{2}\left(T_{c}\right)+r_{1}+\frac{1}{4} T_{c}} \eta^{2}\left(T_{c}, x\right) d x \geq K_{0}|d(\varepsilon)|^{2} c^{\frac{4}{m-1}+\frac{1}{2}} .
$$

Proof of (113). The proof will proceed by a contradiction argument. Indeed, suppose that for any $\alpha>0$ there exists $c>0$ small enough such that (113) does not hold properly, namely

$$
\left\|\eta\left(T_{c}\right)\right\|_{L^{2}\left(x<\bar{\rho}_{2}\left(T_{c}\right)+r_{1}+\frac{1}{4} T_{c}\right)} \leq \alpha|d(\varepsilon)| c^{\frac{2}{m-1}+\frac{1}{4}} .
$$

Replacing

$$
u\left(T_{c}, x\right)=Q_{\bar{c}_{1}(T)}\left(x-r_{1}-\bar{\rho}_{1}\left(T_{c}\right)\right)+Q_{\bar{c}_{2}\left(T_{c}\right)}\left(x-r_{1}-\bar{\rho}_{2}\left(T_{c}\right)\right)+\eta\left(T_{c}, x\right)
$$

in (102), we find

$$
\begin{aligned}
& \|\left[Q_{\bar{c}_{1}\left(T_{c}\right)}\left(\cdot-r_{1}-\bar{\rho}_{1}\left(T_{c}\right)\right)-Q\left(\cdot-r_{1}\right)\right]+\left[Q_{\bar{c}_{2}\left(T_{c}\right)}\left(\cdot-r_{1}-\bar{\rho}_{2}\left(T_{c}\right)\right)-Q_{c}\left(\cdot-r_{2}\right)\right] \\
& \quad+\eta\left(T_{c}\right)+2 d(\varepsilon)\left(Q_{c}^{2}\right)^{\prime}\left(\cdot-r_{2}\right) \|_{H^{1}(\mathbb{R})} \leq K c^{\frac{2}{m-1}+\frac{1}{4}}\left[c^{\frac{1}{m-1}}+|d(\varepsilon)| c^{\frac{1}{2}}\right]
\end{aligned}
$$


By the decay properties of $Q$, (110) at time $t=T_{c}$ and $r_{1}-r_{2} \geq \frac{1}{2}(1-c) T_{c}$ (see (103)), we obtain

$$
\begin{aligned}
\left\|\left[Q_{\bar{c}_{2}\left(T_{c}\right)}\left(\cdot-r_{1}-\bar{\rho}_{2}\left(T_{c}\right)\right)-Q_{c}\left(\cdot-r_{2}\right)\right]+\eta\left(T_{c}\right)+2 d(\varepsilon)\left(Q_{c}^{2}\right)^{\prime}\left(\cdot-r_{2}\right)\right\|_{L^{2}\left(x<\bar{\rho}_{2}\left(T_{c}\right)+r_{1}+\frac{1}{4} T_{c}\right)} \\
\leq K c^{\frac{2}{m-1}+\frac{1}{4}}\left[c^{\frac{1}{m-1}}+|d(\varepsilon)| c^{\frac{1}{2}}\right] .
\end{aligned}
$$

Then, using (114) and (105),

$$
\begin{aligned}
\|\left[Q_{\bar{c}_{2}\left(T_{c}\right)}\left(\cdot-r_{1}-\bar{\rho}_{2}\left(T_{c}\right)\right)-Q_{c}\left(.-r_{2}\right)\right]+2 d(\varepsilon) & \left(Q_{c}^{2}\right)^{\prime}\left(\cdot-r_{2}\right) \|_{L^{2}\left(x<\bar{\rho}_{2}\left(T_{c}\right)+r_{1}+\frac{1}{4} T_{c}\right)} \\
& \leq c^{\frac{2}{m-1}+\frac{1}{4}}\left[K c^{\frac{1}{2}}+2 \alpha+\frac{K}{\kappa_{0}} c^{\frac{1}{100}}\right]|d(\varepsilon)| .
\end{aligned}
$$

By scaling and translation, and decay of $Q$, we obtain

$$
\begin{aligned}
\left\|\bar{Q}-Q+2 d(\varepsilon) c^{\frac{1}{2}+\frac{1}{m-1}}\left(Q^{2}\right)^{\prime}\right\|_{L^{2}(\mathbb{R})} \leq & c^{\frac{1}{m-1}+\frac{1}{2}}\left[K c^{\frac{1}{2}}+2 \alpha+\frac{K}{\kappa_{0}} c^{\frac{1}{100}}\right]|d(\varepsilon)| \\
& +\left\|\bar{Q}-Q+2 d(\varepsilon) c^{\frac{1}{2}+\frac{1}{m-1}}\left(Q^{2}\right)^{\prime}\right\|_{L^{2}(x>\beta)},
\end{aligned}
$$

where $\bar{Q}(x)=\lambda Q(\mu x-\xi)$, and

$$
\lambda:=\left[\frac{\bar{c}_{2}\left(T_{c}\right)}{c}\right]^{\frac{1}{m-1}}, \quad \mu:=\sqrt{\frac{\bar{c}_{2}\left(T_{c}\right)}{c}},
$$

(do not be confused with $\mu$ of Theorem 1.4), and

$$
\xi=\sqrt{\bar{c}_{2}\left(T_{c}\right)}\left(\bar{\rho}_{2}\left(T_{c}\right)+r_{1}-r_{2}\right), \quad \beta:=\sqrt{c}\left(\frac{1}{4} T_{c}+\bar{\rho}_{2}\left(T_{c}\right)+r_{1}-r_{2}\right) .
$$

Note that from (110) and (111),

$$
\beta \geq \frac{1}{8} \sqrt{c} T_{c} \geq \frac{1}{8} c^{-\frac{1}{100}}, \quad\left\|\bar{Q}-Q+2 d(\varepsilon) c^{\frac{1}{2}+\frac{1}{m-1}}\left(Q^{2}\right)^{\prime}\right\|_{L^{2}(x>\beta)} \leq K c^{10} .
$$

Moreover, note that $\bar{Q}(x)=Q_{\mu}\left(x-\frac{\xi}{\mu}\right)$, and that by (110), we have

$$
|\mu-1| \leq K|d(\varepsilon)| c^{\frac{1}{2}+\frac{1}{m-1}}, \quad|\xi| \leq K|d(\varepsilon)| c^{\frac{1}{2}+\frac{1}{m-1}} .
$$

Expanding $\bar{Q}$ in $\mu-1$, and $\xi / \mu$, and using parity properties, we find

$$
\left\|\xi Q^{\prime}+2 d(\varepsilon) c^{\frac{1}{2}+\frac{1}{m-1}}\left(Q^{2}\right)^{\prime}\right\|_{L^{2}(\mathbb{R})} \leq\left[K c^{\frac{1}{2}}+3 \alpha+\frac{K}{\kappa_{0}} c^{\frac{1}{100}}\right]|d(\varepsilon)| c^{\frac{1}{2}+\frac{1}{m-1}},
$$

so that for some constant $\bar{\xi} \in \mathbb{R}$,

$$
\left\|\bar{\xi} Q^{\prime}+2 d(\varepsilon)\left(Q^{2}\right)^{\prime}\right\|_{L^{2}(\mathbb{R})} \leq\left[K c^{\frac{1}{2}}+4 \alpha+\frac{K}{\kappa_{0}} c^{\frac{1}{100}}\right]|d(\varepsilon)| .
$$

Note that exists $\kappa_{1}>0$, independent of $\varepsilon$ and $c$, such that

$$
\inf _{\bar{\xi} \in \mathbb{R}}\left\|\bar{\xi} Q^{\prime}+2 d(\varepsilon)\left(Q^{2}\right)^{\prime}\right\|_{L^{2}(\mathbb{R})} \geq \kappa_{1}|d(\varepsilon)|,
$$

since $Q^{\prime} \neq \gamma\left(Q^{2}\right)^{\prime}$ for all $\gamma \in \mathbb{R}$. By choosing $\kappa_{0}$ large enough in (105), depending only on $\kappa_{1}$, and $c$ small enough, we find a contradiction for $\alpha$ small. This contradiction proves (113).

Now, we finish the proof of the lower bound by proving the following

Lemma 4.1. There exists $K_{0}>0$ such that

$$
\liminf _{t \rightarrow+\infty}\left\|w^{+}(t)\right\|_{H_{c}^{1}(\mathbb{R})} \geq K_{0}|d(\varepsilon)| c^{\frac{3}{4}+\frac{2}{m-1}} .
$$

Note that (115) combined with (56)-(57) prove the lower bound in (26). Thus, we are now reduced to prove (115). 
Proof. We argue by contradiction. Assume that for any $\alpha>0$, there exist arbitrarily large $T_{0}$ and $c$ arbitrarily close to 0 such that

$$
\left\|w^{+}\left(T_{0}\right)\right\|_{H_{c}^{1}(\mathbb{R})} \leq \alpha|d(\varepsilon)| c^{\frac{3}{4}+\frac{2}{m-1}} .
$$

By (112), we can choose $T_{0}>T_{c}$ large enough so that

$$
\left\|\eta\left(T_{0}\right)\right\|_{H_{c}^{1}\left(x<m\left(T_{0}\right)+\frac{T_{0}}{4}\right)} \leq 2 \alpha|d(\varepsilon)| c^{\frac{3}{4}+\frac{2}{m-1}} .
$$

Here $m(t):=r_{1}+\frac{1}{2}\left(\bar{\rho}_{1}(t)+\bar{\rho}_{2}(t)\right)$ is the middle point between the two solitons at time $t$.

We need to estimate some local in space conservation laws. For this reason we introduce a sort of cutoff function supported on the small soliton. Let

$$
\begin{aligned}
& \psi(x)=\frac{2}{\pi} \arctan (\exp (x / \kappa)), \quad \text { so that } \quad \lim _{-\infty} \psi=0, \lim _{\infty} \psi=1, \text { and for all } x \in \mathbb{R}, \\
& \psi(-x)=1-\psi(x), \quad \psi^{\prime}(x)=\frac{1}{\pi \kappa \cosh (x / \kappa)}, \quad\left|\psi^{\prime \prime \prime}(x)\right| \leq \frac{1}{\kappa^{2}}\left|\psi^{\prime}(x)\right| .
\end{aligned}
$$

Let

We set

$$
a:=\frac{E\left(Q_{\bar{c}\left(T_{0}\right)}\right)-E\left(Q_{\bar{c}\left(T_{c}\right)}\right)}{M\left(Q_{\bar{c}\left(T_{c}\right)}\right)-M\left(Q_{\bar{c}\left(T_{0}\right)}\right)}
$$

$$
\begin{aligned}
\mathcal{G}(t) & :=\frac{1}{2} a \int_{\mathbb{R}} u^{2}(t, x)(1-\psi(x-m(t))) d x+\frac{1}{2} \int_{\mathbb{R}}\left(u_{x}^{2}-2 F(u)\right)(t, x)(1-\psi(x-m(t))) d x \\
& =a M(u(t))+E(u(t))-\left(a \mathcal{M}_{1}(t)+\mathcal{E}_{1}(t)\right),
\end{aligned}
$$

where

$$
\mathcal{M}_{1}(t):=\frac{1}{2} \int_{\mathbb{R}} u^{2}(t, x) \psi(x-m(t)) d x, \quad \mathcal{E}_{1}(t):=\frac{1}{2} \int_{\mathbb{R}}\left(u_{x}^{2}-2 F(u)\right)(t, x) \psi(x-m(t)) d x .
$$

We claim the following results on $m(t), a$ and $\mathcal{G}(t)$.

Claim 7. The following estimates hold

$$
\frac{1}{2} \leq m^{\prime}(t) \leq \frac{3}{2}
$$

and for a positive constant $k_{m}$,

$$
a=k_{m} c+o(c)
$$

(Here $o(c)$ means $\left|c^{-1} o(c)\right| \rightarrow 0$ as $c \rightarrow 0$.)

Proof. To prove (119), it is enough to consider Lemma 3.4 on the interval $\left[T_{c}, T_{0}\right]$ to have

$$
m^{\prime}(t) \geq 1-\frac{1}{10} \geq \frac{1}{2} ; \quad m^{\prime}(t) \leq 1+\frac{1}{10} \leq \frac{3}{2} .
$$

Let us now treat (120). It is easy to show that

$$
M\left(Q_{c}\right)=c^{\frac{2}{m-1}-\frac{1}{2}} \int_{\mathbb{R}} Q^{2}+o\left(c^{\frac{2}{m-1}-\frac{1}{2}}\right) .
$$

On the other hand,

$$
E\left(Q_{c}\right)=\frac{1}{2} c^{\frac{2}{m-1}+\frac{1}{2}}\left[\int_{\mathbb{R}} Q^{\prime 2}-\frac{2}{m+1} \int_{\mathbb{R}} Q^{m+1}\right]+o\left(c^{\frac{m+1}{m-1}+\frac{1}{2}}\right) .
$$

Thus, from (110) and the fact that $E(Q)<0$, a Taylor expansion and L'Hopital rule gives

$$
a=-\left.\frac{\partial_{c} E\left(Q_{c}\right)}{\partial_{c} M\left(Q_{c}\right)}\right|_{c=\bar{c}\left(T_{c}\right)}+O\left(\left|\bar{c}\left(T_{0}\right)-\bar{c}\left(T_{c}\right)\right|\right)=-\frac{E(Q)}{M(Q)} \bar{c}\left(T_{c}\right)+o(c)=k_{m} c+o(c) .
$$

where $k_{m}:=-\frac{E(Q)}{M(Q)}>0$ is a constant depending on $m$.

Lemma 4.2. For $0<c<c_{0}$ small enough,

$$
\mathcal{G}\left(T_{c}\right)-\mathcal{G}\left(T_{0}\right) \leq K c^{10} .
$$

Proof. We will need the following 
Claim 8. Define $h:=u_{x x}+f(u)$, such that $u_{t}=-h_{x}$. Then

$$
\mathcal{M}_{1}^{\prime}(t)=-\frac{3}{2} a \int_{\mathbb{R}} u_{x}^{2} \psi^{\prime}+\frac{a}{2} \int_{\mathbb{R}} u^{2}\left(\psi^{\prime \prime \prime}-m^{\prime} \psi^{\prime}\right)+a \int_{\mathbb{R}}(u f(u)-F(u)) \psi^{\prime},
$$

and

$$
\begin{aligned}
\mathcal{E}_{1}^{\prime}(t)=- & \frac{3}{2} \int_{\mathbb{R}} h^{2} \psi^{\prime}-\frac{1}{2} \int_{\mathbb{R}} u_{x}^{2}\left(m^{\prime} \psi^{\prime}-\psi^{\prime \prime \prime}\right)+\int_{\mathbb{R}} F(u)\left(m^{\prime} \psi^{\prime}+\psi^{\prime \prime \prime}\right) \\
& +\int_{\mathbb{R}} f^{2}(u) \psi^{\prime}-\int_{\mathbb{R}} u_{x}^{2} f^{\prime}(u) \psi^{\prime} .
\end{aligned}
$$

Proof. A direct computation, see for example [26].

Now, we follow the proof contained in Appendix D, [26]. From above Claim, we have

$$
\begin{aligned}
\mathcal{G}^{\prime}(t)= & \frac{3}{2} \int_{\mathbb{R}} h^{2} \psi^{\prime}+\frac{1}{2} \int_{\mathbb{R}} u_{x}^{2}\left(m^{\prime} \psi^{\prime}-\psi^{\prime \prime \prime}+3 a \psi^{\prime}\right)+\frac{a}{2} \int_{\mathbb{R}} u^{2}\left(m^{\prime} \psi^{\prime}-\psi^{\prime \prime \prime}\right) \\
& -a \int_{\mathbb{R}}(u f(u)-F(u)) \psi^{\prime}-\int_{\mathbb{R}} F(u)\left(m^{\prime} \psi^{\prime}+\psi^{\prime \prime \prime}\right)-\int_{\mathbb{R}} f^{2}(u) \psi^{\prime}+\int_{\mathbb{R}} u_{x}^{2} f^{\prime}(u) \psi^{\prime} .
\end{aligned}
$$

From Claim 7 we choose $\kappa>0$ large enough such that $m^{\prime} \psi^{\prime}-\psi^{\prime \prime \prime} \geq \frac{1}{4} \psi^{\prime}$. From here,

$$
\frac{3}{2} \int_{\mathbb{R}} h^{2} \psi^{\prime}+\frac{1}{2} \int_{\mathbb{R}} u_{x}^{2}\left(m^{\prime} \psi^{\prime}-\psi^{\prime \prime \prime}+3 a \psi^{\prime}\right)+\frac{a}{2} \int_{\mathbb{R}} u^{2}\left(m^{\prime} \psi^{\prime}-\psi^{\prime \prime \prime}\right) \geq \frac{c}{4} \int_{\mathbb{R}}\left(u_{x}^{2}+u^{2}\right) \psi^{\prime}
$$

Let us consider now the nonlinear terms in the second row of $\mathcal{G}^{\prime}(t)$. For this, let

$$
I:=\left[r_{1}+\bar{\rho}_{2}(t)+\frac{1}{8} T_{c}, r_{1}+\bar{\rho}_{1}(t)-\frac{1}{8} T_{c}\right]
$$

an interval between the two solitons. We have two cases: $x \in I$ and $x \notin I$.

In the first case, from (109) we have for all $t \geq T_{c}$

$$
|u(t)| \leq\left|Q_{\bar{c}_{1}}\right|(t)+\left|Q_{\bar{c}_{2}}\right|(t)+|\eta|(t) \leq K|d(\varepsilon)| c^{\frac{2}{m-1}-\frac{1}{4}}
$$

Thus,

$$
\begin{aligned}
& \left|-a \int_{I}(u f(u)-F(u)) \psi^{\prime}-\int_{I} F(u)\left(m^{\prime} \psi^{\prime}+\psi^{\prime \prime \prime}\right)-\int_{I} f^{2}(u) \psi^{\prime}+\int_{I} u_{x}^{2} f^{\prime}(u) \psi^{\prime}\right| \\
& \leq K\left[\|u(t)\|_{L^{\infty}(I)}^{m-1}+\|u(t)\|_{L^{\infty}(I)}^{2(m-1)}\right] \int_{\mathbb{R}}\left(u^{2}+u_{x}^{2}\right) \psi^{\prime} \\
& \leq K|d(\varepsilon)|^{m-1} c^{2-\frac{1}{4}(m-1)}\left[|d(\varepsilon)|^{m-1} c^{2-\frac{1}{4}(m-1)}+1\right] \int_{\mathbb{R}}\left(u^{2}+u_{x}^{2}\right) \psi^{\prime} \\
& \leq K c^{\frac{3}{2}} \int_{\mathbb{R}}\left(u^{2}+u_{x}^{2}\right) \psi^{\prime} .
\end{aligned}
$$

In the second case, we have $|x-m(t)| \geq \frac{t}{4}$ and thus $\psi^{\prime}(x-m(t)) \leq K e^{-\gamma t}$, with $\gamma>0$ a fixed constant. From here,

$\left|-a \int_{x \notin I}(u f(u)-F(u)) \psi^{\prime}-\int_{x \notin I} F(u)\left(m^{\prime} \psi^{\prime}+\psi^{\prime \prime \prime}\right)-\int_{x \notin I} f^{2}(u) \psi^{\prime}+\int_{x \notin I} u_{x}^{2} f^{\prime}(u) \psi^{\prime}\right| \leq K e^{-\gamma t}$.

In conclusion, putting together above estimates, we get for all $t \in\left[T_{c}, T_{0}\right]$,

$$
\mathcal{G}^{\prime}(t) \geq-K e^{-\gamma t},
$$

and after integration we obtain the desired result. The proof is now complete.

Now, define

We have the

$$
\mathcal{H}(t):=\int_{\mathbb{R}}\left[a \eta^{2}+\eta_{x}^{2}-f^{\prime}\left(R_{2}\right) \eta^{2}\right](1-\psi)
$$

Lemma 4.3. For $0<c<c_{0}$ small enough, 
(1) Small variation:

$$
\begin{aligned}
\mathcal{G}\left(T_{c}\right)-\mathcal{G}\left(T_{0}\right)=\frac{1}{2} & \left(\mathcal{H}\left(T_{c}\right)-\mathcal{H}\left(T_{0}\right)\right)+O\left(\alpha^{2}|d(\varepsilon)|^{3} c^{\frac{6}{m-1}+\frac{1}{4}} c^{\frac{m-2}{m-1}}\right) \\
& +O\left(|d(\varepsilon)| c^{\frac{2}{m-1}-\frac{1}{4}} c^{\frac{m-2}{m-1}} \int_{\mathbb{R}} \eta^{2}\left(T_{c}\right)(1-\psi)\right)+O\left(c^{10}\right),
\end{aligned}
$$

(2) Coercivity:

$$
\mathcal{H}(t) \geq \sigma_{0} \int_{\mathbb{R}}\left[c \eta^{2}+\eta_{x}^{2}\right](t, x)(1-\psi) d x .
$$

for some $\sigma_{0}>0$ independent of $c$.

Proof. Let us first prove (122). We replace $u=R_{1}+R_{2}+\eta$ in the definition of $\mathcal{G}$. We obtain

$$
\begin{aligned}
\mathcal{M}(t) & =\frac{1}{2} \int_{\mathbb{R}}\left(R_{1}+R_{2}+\eta\right)^{2}(1-\psi) \\
& =\frac{1}{2} \int_{\mathbb{R}} R_{2}^{2}(1-\psi)+\int_{\mathbb{R}} \eta R_{2}(1-\psi)+\frac{1}{2} \int_{\mathbb{R}} \eta^{2}(1-\psi)+O\left(c^{10}\right) .
\end{aligned}
$$

Here we have used the estimate for $t \geq T_{c}$

$$
\left|\int_{\mathbb{R}} R_{1}(t)(1-\psi)\right| \leq K e^{-\frac{1}{2} t} \leq K c^{10},
$$

among other similar estimates.

In the same way,

$$
\frac{1}{2} \int_{\mathbb{R}} u_{x}^{2}(1-\psi)=\frac{1}{2} \int_{\mathbb{R}}\left(R_{2}\right)_{x}^{2}(1-\psi)+\int_{\mathbb{R}} \eta_{x}\left(R_{2}\right)_{x}(1-\psi)+\frac{1}{2} \int_{\mathbb{R}} \eta_{x}^{2}(1-\psi)+O\left(c^{10}\right) .
$$

Finally, using the character exponentially decreasing of $R_{1}$ where $1-\psi$ is away from zero,

$$
\begin{aligned}
\int_{\mathbb{R}} F(u)(1-\psi)= & \int_{\mathbb{R}} F\left(R_{1}+R_{2}+\eta\right)(1-\psi) \\
= & \int_{\mathbb{R}}\left[F\left(R_{1}+R_{2}+\eta\right)-F\left(R_{2}+\eta\right)\right](1-\psi) \\
& +\int_{\mathbb{R}}\left[F\left(R_{2}+\eta\right)-F\left(R_{2}\right)-f\left(R_{2}\right) \eta-\frac{1}{2} f^{\prime}\left(R_{2}\right) \eta^{2}\right](1-\psi) \\
& +\int_{\mathbb{R}}\left[F\left(R_{2}\right)+f\left(R_{2}\right) \eta+\frac{1}{2} f^{\prime}\left(R_{2}\right) \eta^{2}\right](1-\psi) \\
= & \int_{\mathbb{R}}\left[f\left(R_{2}+\eta\right) R_{1}+O\left(R_{1}^{2}\right)\right](1-\psi)+O\left(\left\|R_{2}\right\|_{L^{\infty}(\mathbb{R})}^{m-2} \int_{\mathbb{R}}|\eta(t)|^{3}(1-\psi)\right) \\
& +\int_{\mathbb{R}}\left[F\left(R_{2}\right)+f\left(R_{2}\right) \eta+\frac{1}{2} f^{\prime}\left(R_{2}\right) \eta^{2}\right](1-\psi) \\
= & \int_{\mathbb{R}}\left[F\left(R_{2}\right)+f\left(R_{2}\right) \eta+\frac{1}{2} f^{\prime}\left(R_{2}\right) \eta^{2}\right](1-\psi) \\
& +O\left[\left\|R_{2}\right\|_{L^{\infty}(\mathbb{R})}^{m-2}\|\eta(t)\|_{H^{1}} \int_{\mathbb{R}} \eta^{2}(t)(1-\psi)\right]+O\left(c^{10}\right) .
\end{aligned}
$$

From this,

$$
\mathcal{G}(t)=\mathcal{G}\left[R_{2}\right](t)+\mathcal{H}(t)+O\left[\left\|R_{2}\right\|_{L^{\infty}(\mathbb{R})}^{m-2}\|\eta(t)\|_{H^{1}} \int_{\mathbb{R}} \eta^{2}(t)(1-\psi)\right]+O\left(c^{10}\right) .
$$

Putting together these estimates, using the value of $a$, evaluating at times $t=T_{c}$ and $t=T_{0}$ and using (117) and (110), we obtain the desired result.

The proof of (123) is standard, see e.g. 223] Appendix B.3. 
Combining Lemmas 4.2 and 4.3 , we find

$$
\begin{aligned}
\int_{\mathbb{R}}\left[c \eta^{2}+\eta_{x}^{2}\right]\left(T_{c}\right)(1-\psi) \leq & K \mathcal{H}\left(T_{c}\right) \\
\leq & K \mathcal{H}\left(T_{0}\right)+K|d(\varepsilon)| c^{\frac{1}{m-1}+\frac{3}{4}} \int_{\mathbb{R}} \eta^{2}\left(T_{c}\right)(1-\psi) \\
& \quad+K \alpha^{2}|d(\varepsilon)|^{3} c^{\frac{5}{m-1}+\frac{5}{4}}+O\left(c^{10}\right)+K\left(\mathcal{G}\left(T_{c}\right)-\mathcal{G}\left(T_{0}\right)\right) \\
\leq & K \alpha^{2}|d(\varepsilon)|^{2} c^{\frac{4}{m-1}+\frac{3}{2}}+K \alpha^{2}|d(\varepsilon)|^{3} c^{\frac{5}{m-1}+\frac{5}{4}}+K c^{10} .
\end{aligned}
$$

The last inequality is consequence of

therefore the term

$$
|d(\varepsilon)| c^{\frac{1}{m-1}+\frac{3}{4}} \ll \frac{c}{K}, \quad m=2,3 \text { and } 4 ;
$$

$$
K|d(\varepsilon)| c^{\frac{1}{m-1}+\frac{3}{4}} \int_{\mathbb{R}} \eta^{2}\left(T_{c}\right)(1-\psi)
$$

can be sent to the left hand side of (124). Using (113) we finally get

$$
|d(\varepsilon)|^{2} c^{\frac{4}{m-1}+\frac{3}{2}} \leq K \alpha^{2}|d(\varepsilon)|^{2} c^{\frac{4}{m-1}+\frac{3}{2}}+K \alpha^{2}|d(\varepsilon)|^{3} c^{\frac{5}{m-1}+\frac{5}{4}} .
$$

But this estimate is a contradiction for $\alpha>0$ small enough and $0<c<c_{0}$ small enough (it is enough to put $\varepsilon$ even smaller). The proof of Claim 4.1 is now complete.

5. Lower bounds on the parameters. We finally prove (27). This result is a consequence of Theorem [1.2, (17), (18) and (19), see also 25) for the proof. Indeed, from (17) and (26), we have

$$
\frac{1}{K}|d(\varepsilon)|^{2} c^{\frac{3}{2}+\frac{4}{m-1}} \leq 2 E^{+}+c M^{+} \leq \frac{1}{K}|d(\varepsilon)|^{2} c^{\frac{1}{2}+\frac{4}{m-1}} .
$$

The final conclusion follows from (18), (19) and (56)-(57).

This finishes the proof of the Theorem 1.4.

\section{Appendix A. Proof of Proposition 2.2}

The proof is similar to Proposition 2.2 in [25] and Appendix in 24]. The main difference consists in the fact that we need to know explicitly all linear systems up to order $m+1$ to show the nonexistence of growing solutions. We will discard several trivial terms by using the property (IP). For this purpose it is better to state an improved version of Proposition 2.2. Before that we introduce a useful notation.

Definition A.1. Consider $f, g: \mathbb{R} \rightarrow \mathbb{R}$ given functions. We say that $f=g \bmod \mathcal{Y}$ if there exists $h \in \mathcal{Y}$ such that $f=g+h$.

In our case, this definition will be useful to discard localized functions in the source terms. Indeed,

Proposition A.1 (Decomposition of $S(\tilde{u})$, improved version). Assume that $f$ is of class $C^{m+2}$. Let

$$
\mathcal{L} w=-w_{y y}+w-f^{\prime}(Q) w .
$$

Then,

$$
\begin{aligned}
S[\tilde{u}](t, x)= & \sum_{(k, l) \in \Sigma_{m}} c^{l} Q_{c}^{k}\left(y_{c}\right)\left[a_{k, l}(-3 Q+2 f(Q))^{\prime}(y)-\left(\mathcal{L} A_{k, l}\right)^{\prime}(y)+F_{k, l}(y)\right] \\
& +\sum_{(k, l) \in \Sigma_{m}} c^{l}\left(Q_{c}^{k}\right)^{\prime}\left(y_{c}\right)\left[a_{k, l}\left(-3 Q^{\prime \prime}\right)(y)+\left(3 A_{k, l}^{\prime \prime}+f^{\prime}(Q) A_{k, l}\right)(y)-\left(\mathcal{L} B_{k, l}\right)^{\prime}(y)+G_{k, l}(y)\right] \\
& +\mathcal{E}(t, x)
\end{aligned}
$$

where $F_{k, l}, G_{k, l}$ and $\mathcal{E}$ satisfy, for any $(k, l) \in \Sigma_{m}$,

(i) Dependence property of $F_{k, l}$ and $G_{k, l}$ : The expressions of $F_{k, l}$ and $G_{k, l}$ depend only on $\left(a_{k^{\prime}, l^{\prime}}\right),\left(A_{k^{\prime}, l^{\prime}}\right),\left(B_{k^{\prime}, l^{\prime}}\right)$ for $\left(k^{\prime}, l^{\prime}\right)<(k, l)$. 
(ii) Parity property of $F_{k, l}$ and $G_{k, l}$ : Assume that for any $\left(k^{\prime}, l^{\prime}\right)$ such that $\left(k^{\prime}, l^{\prime}\right)<(k, l)$ $A_{k^{\prime}, l^{\prime}}$ is even and $B_{k^{\prime}, l^{\prime}}$ is odd, then $F_{k, l}$ is odd and $G_{k, l}$ is even.

(iii) Explicit source terms: We have $F_{1,0}=\left(f^{\prime}(Q)\right)^{\prime}$ and $G_{1,0}=f^{\prime}(Q)$,

$$
\begin{aligned}
F_{2,0}=- & \left(3 A_{1,0}^{\prime}+3 B_{1,0}^{\prime \prime}+f^{\prime}(Q) B_{1,0}\right)+\frac{1}{2}\left(f^{\prime \prime}(Q)\left(2 A_{1,0}+A_{1,0}^{2}\right)\right)^{\prime} \\
& -a_{1,0}\left(3 A_{1,0}^{\prime \prime}-Q+f^{\prime}(Q)\left(1+A_{1,0}\right)\right)^{\prime}+3 a_{1,0}^{2} Q^{(3)}+\frac{1}{2}\left(f^{\prime \prime}(Q)-2\right)^{\prime},
\end{aligned}
$$

and

$$
\begin{aligned}
G_{2,0}= & \frac{1}{2}\left(f^{\prime \prime}(Q)-2\right)-\left(A_{1,0}+3 B_{1,0}^{\prime}\right)+\frac{1}{2} f^{\prime \prime}(Q)\left(2 A_{1,0}+A_{1,0}^{2}\right)+\frac{3}{2} a_{1,0}^{2} Q^{\prime \prime} \\
& -\frac{1}{2} a_{1,0}\left(9 A_{1,0}^{\prime}+3 B_{1,0}^{\prime \prime}+f^{\prime}(Q) B_{1,0}\right)^{\prime}+\frac{1}{2}\left(f^{\prime \prime}(Q)\left(B_{1,0}+A_{1,0} B_{1,0}\right)\right)^{\prime} .
\end{aligned}
$$

for the case $m=2$, and

$$
F_{2,0}=\left(\frac{1}{2} f^{\prime \prime}(Q)\left(1+A_{1,0}\right)^{2}\right)^{\prime}+3 a_{1,0}^{2} Q^{(3)}-a_{1,0}\left(f^{\prime}(Q)+3 A_{1,0}^{\prime \prime}+f^{\prime}(Q) A_{1,0}\right)^{\prime}
$$

and

$$
\begin{aligned}
G_{2,0}= & \frac{1}{2} f^{\prime \prime}(Q)\left(1+A_{1,0}\right)^{2}+\frac{3}{2} a_{1,0}^{2} Q^{\prime \prime}-\frac{1}{2} a_{1,0}\left(9 A_{1,0}^{\prime}+3 B_{1,0}^{\prime \prime}+f^{\prime}(Q) B_{1,0}\right)^{\prime} \\
& +\frac{1}{2}\left(f^{\prime \prime}(Q)\left(1+A_{1,0}\right) B_{1,0}\right)^{\prime} .
\end{aligned}
$$

in the case $m=3$. If property (IP) holds for $(k, l)=(1,0)$, then each term above is in $\mathcal{Y}$.

(iv) Explicit high order source terms modulo $\mathcal{Y}$ : Suppose property (IP) holds for $(k, l) \in \Sigma_{m}$ with $k+l \leq 2$. Then, for the quadratic case,

$$
F_{1,2}, G_{1,2}, F_{2,1} \text { and } F_{3,0} \in \mathcal{Y} ; \quad G_{3,0}=-\frac{2}{3}\left(B_{1,0}^{2}+2 A_{2,0}\right) \quad \bmod \mathcal{Y},
$$

and

$$
G_{2,1}=B_{1,0}^{2}+A_{1,1}+3 A_{2,0} \bmod \mathcal{Y}
$$

For the cubic case,

$$
F_{3,0}, G_{3,0}, F_{2,1}, F_{4,0} \text { and } G_{4,0} \in \mathcal{Y}, \quad G_{2,1}=3 A_{2,0} \quad \bmod \mathcal{Y} .
$$

(v) Improved estimate on $\mathcal{E}$ : Suppose in addition that property (IP) holds for any $(k, l) \in \Sigma_{m}$, then for all $j=0,1,2$

$$
\left\|\partial_{x}^{j} \mathcal{E}(t, x)\right\|_{H^{1}(\mathbb{R})} \leq K c^{\frac{3}{4}+\frac{3}{m-1}} .
$$

Proof. Expansion (126), and items (i) and (ii) were proven in [25], so in what follows we deal with (iii)-(v). For this it is necesary to improve the computation done in [25].

We start with an important lemma concerning the algebra of $Q_{c}$.

Lemma A.2 (Properties of $Q_{c}$, see Lemma 2.1 in [25]). Suppose $0<c \leq 1,0<\varepsilon \leq \varepsilon_{0}$ small, $k \in\left\{1, \ldots, k_{0}\right\}$, and $m=2,3$. Then

(1) There exists a positive constant $K=K(\varepsilon)>0$ such that

$$
\frac{1}{K} c^{\frac{1}{m-1}} e^{-\sqrt{c}|x|} \leq Q_{c}(x) \leq K c^{\frac{1}{m-1}} e^{-\sqrt{c}|x|}, \quad\left|Q_{c}^{\prime}(x)\right| \leq K c^{\frac{1}{m-1}+\frac{1}{2}} e^{-\sqrt{c}|x|} .
$$

(2) For $F$ defined in (9) and any $k \geq 1$,

$$
\begin{gathered}
Q_{c}^{\prime \prime}=c Q_{c}-f\left(Q_{c}\right), \quad Q_{c}^{\prime 2}=c Q_{c}^{2}-2 F\left(Q_{c}\right) . \\
\left(Q_{c}^{k}\right)^{\prime \prime}=c k^{2} Q_{c}^{k}-2 k(k-1) Q_{c}^{k-2} F\left(Q_{c}\right)-k f\left(Q_{c}\right) Q_{c}^{k-1} .
\end{gathered}
$$


We recall the notation introduced in Subsection (2.1):

$$
S[\tilde{u}]=\tilde{u}_{t}+\left(\tilde{u}_{x x}-\tilde{u}+f(\tilde{u})\right)_{x} .
$$

We easily verify that

where (we omit the dependence on $t, x$ )

$$
S[\tilde{u}]=\mathbf{I}+\mathbf{I I}+\mathbf{I I I}+\mathbf{I V},
$$

$$
\mathbf{I}:=S[R], \quad \mathbf{I I}:=\left(f\left(R+R_{c}\right)-f(R)-f\left(R_{c}\right)\right)_{x},
$$

and

$$
\begin{aligned}
& \mathcal{L}=-\partial_{x}^{2}+1-f^{\prime}(Q), \\
& \mathbf{I I I}=\mathbf{I I I}(W):=W_{t}-(\mathcal{L} W)_{x}, \\
& \mathbf{I V}:=\left\{f\left(R+R_{c}+W\right)-f\left(R+R_{c}\right)-f(R) W\right\}_{x} .
\end{aligned}
$$

Since $Q_{c}\left(y_{c}\right)$ is a solution to (11), we have $S\left(Q_{c}\right)=0$.

Claim 9. Let $A=A(y)$ and $q=q\left(y_{c}\right)$ be $C^{3}$-functions with $y, y_{c}$ defined in Section 2.1, Then

$$
\begin{aligned}
\mathbf{I I I}(A q)= & -q(\mathcal{L} A)^{\prime}+q^{\prime}\left(3 A^{\prime \prime}+f^{\prime}(Q) A\right) \\
& +q\left(-3 \beta A^{(3)}-\beta A^{\prime}-3 \beta_{x} A^{\prime \prime}-A^{\prime} \beta_{x x}+\beta A^{\prime}-\beta\left(f^{\prime}(Q) A\right)^{\prime}\right) \\
& +q\left(3 \beta^{2} A^{(3)}+3 \beta \beta_{x} A^{\prime \prime}-\beta^{3} A^{(3)}+c \beta A^{\prime}\right) \\
& +q^{\prime}\left(-c A-6 A^{\prime \prime} \beta-3 A^{\prime} \beta_{x}+3 A^{\prime \prime} \beta^{2}\right) \\
& +q^{\prime \prime}\left(3 A^{\prime}-3 A^{\prime} \beta\right)+A q^{(3)} .
\end{aligned}
$$

Proof. Direct differentiation, see [25], Proposition 2.2.

Claim 10. Recall from (31),

$$
\beta=\sum_{(k, l) \in \Sigma_{m}} a_{k, l} c^{l} Q_{c}^{k}\left(y_{c}\right)
$$

Then, for some fixed numbers $\hat{a}_{k, l}^{1}, \hat{a}_{k, l}^{2}, \bar{a}_{k, l}, \tilde{a}_{k, l}$ with $(k, l) \in \Sigma_{m}$, depending only on $a_{k^{\prime}, l^{\prime}}$ with $\left(k^{\prime}, l^{\prime}\right) \leq(k, l)$, we have

$$
\left\{\begin{array}{l}
\beta_{x}=\sum_{(k, l) \in \Sigma_{m}} a_{k, l} c^{l}\left(Q_{c}^{k}\right)^{\prime}\left(y_{c}\right), \\
\beta_{x x}=\sum_{\substack{(k, l) \in \Sigma_{m} \\
l \geq 1}} \hat{a}_{k, l}^{1} c^{l} Q_{c}^{k}\left(y_{c}\right)+\sum_{\substack{(k, l) \in \Sigma_{m} \\
k \geq m}} \hat{a}_{k, l}^{2} c^{l} Q_{c}^{k}\left(y_{c}\right)+O\left(Q_{c}^{5}+c Q_{c}^{3}\right), \\
\beta^{2}=\sum_{\substack{(k, l) \in \Sigma_{m} \\
k \geq 2}} \bar{a}_{k, l} c^{l} Q_{c}^{k}\left(y_{c}\right)+O\left(Q_{c}^{5}+c Q_{c}^{3}\right) \\
\left(\beta^{2}\right)_{x}=\sum_{\substack{(k, l) \in \Sigma_{m} \\
k \geq 2}} \bar{a}_{k, l} c^{l}\left(Q_{c}^{k}\right)^{\prime}\left(y_{c}\right)+O\left(Q_{c}^{5}+c Q_{c}^{3}\right), \text { and } \\
\beta^{3}=\sum_{\substack{(k, l) \in \Sigma_{m} \\
k \geq 3}} \tilde{a}_{k, l} c^{l} Q_{c}^{k}\left(y_{c}\right)+O\left(Q_{c}^{5}+c Q_{c}^{3}\right) .
\end{array}\right.
$$

Proof. The proof follows by elementary calculations from (31).

In the next lemmas, we expand the terms in (130).

Lemma A.3.

$$
\begin{aligned}
\mathbf{I}= & \sum_{(k, l) \in \Sigma_{m}} c^{l}\left[Q_{c}^{k}\left(y_{c}\right) a_{k, l}(2 f(Q)-3 Q)^{\prime}(y)+\left(Q_{c}^{k}\right)^{\prime}\left(y_{c}\right)\left(-3 a_{k, l} Q^{\prime \prime}(y)\right)\right] \\
& +\sum_{(k, l) \in \Sigma_{m}} c^{l}\left(Q_{c}^{k}\left(y_{c}\right) F_{k, l}^{I}(y)+\left(Q_{c}^{k}\right)^{\prime}\left(y_{c}\right) G_{k, l}^{I}(y)\right)+c^{3} O\left(Q_{c}\left(y_{c}\right)\right),
\end{aligned}
$$

where

$$
\begin{aligned}
& F_{1,0}^{I}=G_{1,0}^{I}=F_{1,1}^{I}=G_{1,1}^{I}=0, \\
& F_{2,0}^{I}=3 a_{1,0}^{2} Q^{(3)}+a_{1,0} Q^{\prime} \delta_{m 2}, \quad G_{2,0}^{I}=\frac{3}{2} a_{1,0}^{2} Q^{\prime \prime},
\end{aligned}
$$

and for all $(k, l) \in \Sigma_{m}, F_{k, l}^{I} \in \mathcal{Y}$ is odd, $G_{k, l}^{I} \in \mathcal{Y}$ is even and depend only on $a_{k^{\prime}, l^{\prime}}$ for $\left(k^{\prime}, l^{\prime}\right)<$ $(k, l)$. 
Proof of Lemma A.3. We have (here' denotes derivative with respect to $y$ )

$$
\begin{aligned}
\mathbf{I} & =R_{t}+\left(R_{x x}-R+f(R)\right)_{x} \\
& =-(1-c) \beta Q^{\prime}+(f(Q))^{\prime}(1-\beta)-Q^{\prime}(1-\beta)+\left(Q^{\prime \prime}(1-\beta)^{2}-Q^{\prime} \beta_{x}\right)_{x} \\
& =\left(Q^{\prime \prime}-Q+f(Q)\right)^{\prime}+Q^{(3)}\left(-3 \beta+3 \beta^{2}-\beta^{3}\right)-3 Q^{\prime \prime}\left(\beta_{x}-\beta \beta_{x}\right)-\beta_{x x} Q^{\prime}-\beta(f(Q))^{\prime}+c \beta Q^{\prime} \\
& =-\left[3 \beta Q^{(3)}+3 Q^{\prime \prime} \beta_{x}+\beta(f(Q))^{\prime}\right]+3 \beta^{2} Q^{(3)}+3 \beta \beta_{x} Q^{\prime \prime}-\beta_{x x} Q^{\prime}+c \beta Q^{\prime}-\beta^{3} Q^{(3)} .
\end{aligned}
$$

Hence using Claim 10, we obtain

$$
\begin{aligned}
\mathbf{I} & =a_{1,0}(2 f(Q)-3 Q)^{\prime} Q_{c}\left(y_{c}\right)+a_{1,0}\left(-3 Q^{\prime \prime}\right) Q_{c}^{\prime}\left(y_{c}\right) \\
& +\left(a_{2,0}(2 f(Q)-3 Q)^{\prime}+3 a_{1,0}^{2} Q^{(3)}+a_{1,0} Q^{\prime} \delta_{m 2}\right) Q_{c}^{2}\left(y_{c}\right) \\
& +\left(a_{2,0}\left(-3 Q^{\prime \prime}\right)+\frac{3}{2} a_{1,0}^{2} Q^{\prime \prime}\right)\left(Q_{c}^{2}\right)^{\prime}\left(y_{c}\right) \\
& +\sum_{k+l=3,4} c^{l}\left(a_{k, l}(2 f(Q)-3 Q)^{\prime}(y) Q_{c}^{k}\left(y_{c}\right)+a_{k, l}\left(-3 Q^{\prime \prime}\right)(y)\left(Q_{c}^{k}\right)^{\prime}\left(y_{c}\right)\right) \\
& +\sum_{k+l=3,4} c^{l}\left(F_{k, l}^{I} Q_{c}^{k}\left(y_{c}\right)+G_{k, l}^{I}\left(Q_{c}^{k}\right)^{\prime}\left(y_{c}\right)\right)+c^{3} O\left(Q_{c}\right),
\end{aligned}
$$

where for all $k+l=3, F_{k, l}^{I} \in \mathcal{Y}$ and $G_{k, l}^{I} \in \mathcal{Y}$, as claimed in the statement of the Lemma.

Lemma A.4.

$$
\mathbf{I I}=\sum_{(k, l) \in \Sigma_{m}} c^{l}\left(Q_{c}^{k}\left(y_{c}\right) F_{k, l}^{I I}(y)+\left(Q_{c}^{k}\right)^{\prime}\left(y_{c}\right) G_{k, l}^{I I}(y)\right)+O\left(Q_{c}^{m+2}\right),
$$

where for all $(k, l) \in \Sigma_{m}$ and for all $p \geq m+1, F_{k, l}^{I I}, G_{k, l}^{I I} \in \mathcal{Y}$ and are odd and even respectively. Moreover, for $m=2$,

$$
\begin{aligned}
& F_{1,0}^{I I}=\left(f^{\prime}(Q)\right)^{\prime}, \quad G_{1,0}^{I I}=f^{\prime}(Q), \quad F_{1,1}^{I I}=G_{1,1}^{I I}=0, \\
& F_{2,0}^{I I}=\left(\frac{1}{2} f^{\prime \prime}(Q)-a_{1,0} f^{\prime}(Q)\right)^{\prime}, \quad G_{2,0}^{I I}=\frac{1}{2} f^{\prime \prime}(Q)-1 .
\end{aligned}
$$

Finally, if $m=3$,

$$
\begin{aligned}
& F_{1,0}^{I I}=\left(f^{\prime}(Q)\right)^{\prime}, \quad G_{1,0}^{I I}=f^{\prime}(Q), \quad F_{1,1}^{I I}=G_{1,1}^{I I}=0, \\
& F_{2,0}^{I I}=\left(\frac{1}{2} f^{\prime \prime}(Q)-a_{1,0} f^{\prime}(Q)\right)^{\prime}, \quad G_{2,0}^{I I}=\frac{1}{2} f^{\prime \prime}(Q) .
\end{aligned}
$$

Proof. First define $\tilde{\mathbf{I I}}:=f\left(R+R_{c}\right)-f(R)-f\left(R_{c}\right)$. Note that

$$
\tilde{\mathbf{I}}=f^{\prime}(R) R_{c}+\frac{1}{2} f^{\prime \prime}(R) R_{c}^{2}+\frac{1}{6} f^{(3)}(R) R_{c}^{3}-f\left(R_{c}\right)+\frac{1}{24} f^{(4)}(R) R_{c}^{4}+O\left(R_{c}^{5}\right),
$$

Thus taking derivative

$$
\begin{aligned}
\mathbf{I I}= & \left(f^{\prime}(Q)\right)^{\prime}(1-\beta) Q_{c}+f^{\prime}(Q) Q_{c}^{\prime}+\frac{1}{2}\left(f^{\prime \prime}(Q)\right)^{\prime}(1-\beta) Q_{c}^{2}+\frac{1}{2} f^{\prime \prime}(Q)\left(Q_{c}^{2}\right)^{\prime}+\frac{1}{6}\left(f^{(3)}(Q)\right)^{\prime}(1-\beta) Q_{c}^{3} \\
& +\frac{1}{6} f^{(3)}(Q)\left(Q_{c}^{3}\right)^{\prime}+\frac{1}{24}\left(f^{(4)}(Q)\right)^{\prime} Q_{c}^{4}+\frac{1}{24} f^{(4)}(Q)\left(Q_{c}^{4}\right)^{\prime}-\left(f\left(Q_{c}\right)\right)^{\prime}+O\left(Q_{c}^{5}\right) .
\end{aligned}
$$

Here we have to identify two different results, depending on the value of $m$. For $m=2$, namely, the quadratic case, we will need only up to third order terms. After replacing the value of $\beta$ given by (132), we will obtain (recall that $p \geq 3$ )

$$
\begin{aligned}
\mathbf{I I}= & \left(f^{\prime}(Q)\right)^{\prime} Q_{c}+f^{\prime}(Q) Q_{c}^{\prime}+\left(\frac{1}{2} f^{\prime \prime}(Q)-a_{1,0} f^{\prime}(Q)\right)^{\prime} Q_{c}^{2}+\left(\frac{1}{2} f^{\prime \prime}(Q)-1\right)\left(Q_{c}^{2}\right)^{\prime}-a_{1,1}\left(f^{\prime}(Q)\right)^{\prime} c Q_{c}^{2} \\
& +\left(\frac{1}{6} f^{(3)}(Q)-\frac{1}{2} a_{1,0} f^{\prime \prime}(Q)-a_{2,0} f^{\prime}(Q)\right)^{\prime} Q_{c}^{3}+\frac{1}{6} f^{(3)}(Q)\left(Q_{c}^{3}\right)^{\prime}-\varepsilon\left(Q_{c}^{p}\right)^{\prime}-\left(f_{1}\left(Q_{c}\right)\right)^{\prime}+O\left(Q_{c}^{4}\right) .
\end{aligned}
$$

It is easy to check that every term depending on $y$ up to order $Q_{c}^{3},\left(Q_{c}^{3}\right)^{\prime}$ is indeed in the class $\mathcal{Y}$. Even in the worst case, $p=3$, we will have the cancelation

$$
\frac{1}{6} f^{(3)}(Q)\left(Q_{c}^{3}\right)^{\prime}-\varepsilon\left(Q_{c}^{p}\right)^{\prime}=\frac{1}{6} f_{1}^{(3)}(Q)\left(Q_{c}^{3}\right)^{\prime},
$$


with $\frac{1}{6} f_{1}^{(3)}(Q) \in \mathcal{Y}$.

Let us consider now the cubic case, $m=3$. The procedure is completely similar, although we must keep the fourth order terms. We start by replacing $\beta$ in (134) and collecting similar terms

$$
\begin{aligned}
\mathbf{I I}= & \left(f^{\prime}(Q)\right)^{\prime}(1-\beta) Q_{c}+f^{\prime}(Q) Q_{c}^{\prime}+\frac{1}{2}\left(f^{\prime \prime}(Q)\right)^{\prime}(1-\beta) Q_{c}^{2}+\frac{1}{2} f^{\prime \prime}(Q)\left(Q_{c}^{2}\right)^{\prime}+\frac{1}{6}\left(f^{(3)}(Q)\right)^{\prime}(1-\beta) Q_{c}^{3} \\
& +\frac{1}{6} f^{(3)}(Q)\left(Q_{c}^{3}\right)^{\prime}+\frac{1}{24}\left(f^{(4)}(Q)\right)^{\prime} Q_{c}^{4}+\frac{1}{24} f^{(4)}(Q)\left(Q_{c}^{4}\right)^{\prime}-\left(f\left(Q_{c}\right)\right)^{\prime}+O\left(Q_{c}^{5}\right) . \\
= & \left(f^{\prime}(Q)\right)^{\prime} Q_{c}+f^{\prime}(Q) Q_{c}^{\prime}+\left(\frac{1}{2} f^{\prime \prime}(Q)-a_{1,0} f^{\prime}(Q)\right)^{\prime} Q_{c}^{2}+\frac{1}{2} f^{\prime \prime}(Q)\left(Q_{c}^{2}\right)^{\prime} \\
& \left(\frac{1}{6} f^{(3)}(Q)-\frac{1}{2} a_{1,0} f^{\prime \prime}(Q)-a_{2,0} f^{\prime}(Q)\right)^{\prime} Q_{c}^{3}+\left(\frac{1}{6} f^{(3)}(Q)-1\right)\left(Q_{c}^{3}\right)^{\prime}-a_{1,1}\left(f^{\prime}(Q)\right)^{\prime} c Q_{c}^{2} \\
& +\left(\frac{1}{24} f^{(4)}(Q)-\frac{1}{6} a_{1,0} f^{(3)}(Q)-\frac{1}{2} a_{2,0} f^{\prime \prime}(Q)-a_{3,0} f^{\prime}(Q)\right)^{\prime} Q_{c}^{4}+\frac{1}{24} f^{(4)}(Q)\left(Q_{c}^{4}\right)^{\prime} \\
& -\left(\varepsilon Q_{c}^{p}+f_{1}\left(Q_{c}\right)\right)^{\prime}+O\left(c Q_{c}^{3}+c Q_{c}^{4}+Q_{c}^{5}\right) .
\end{aligned}
$$

It is straightforward to check that every function depending on $y$ is indeed in $\mathcal{Y}$. The only complicated terms are (note that $p \geq 4$ )

$$
\frac{1}{6} f^{(3)}(Q)-1=\frac{1}{6} p(p-1)(p-2) \varepsilon Q^{p-3}+\frac{1}{6} f_{1}^{(3)}(Q) \in \mathcal{Y},
$$

which is in front of $\left(Q_{c}^{3}\right)^{\prime}$; and for $p=4$, facing $\left(Q_{c}^{4}\right)^{\prime}$ we have

$$
\frac{1}{24} f^{(4)}(Q)-\varepsilon=\varepsilon+\frac{1}{24} f_{1}^{(4)}(Q)-\varepsilon=\frac{1}{24} f_{1}^{(4)}(Q) \in \mathcal{Y} .
$$

\section{Lemma A.5.}

$$
\begin{aligned}
\mathbf{I I I} & =\sum_{(k, l) \in \Sigma_{m}} c^{l}\left(Q_{c}^{k}\left(y_{c}\right)\left(-\mathcal{L} A_{k, l}\right)^{\prime}(y)+\left(Q_{c}^{k}\right)^{\prime}\left(y_{c}\right)\left(\left(-\mathcal{L} B_{k, l}\right)^{\prime}+3 A_{k, l}^{\prime \prime}+f^{\prime}(Q) A_{k, l}\right)(y)\right) \\
& +\sum_{(k, l) \in \Sigma_{m}} c^{l}\left(Q_{c}^{k}\left(y_{c}\right) F_{k, l}^{I I}(y)+\left(Q_{c}^{k}\right)^{\prime}\left(y_{c}\right) G_{k, l}^{I I I}(y)\right)+O\left(Q_{c}^{m+2}\right),
\end{aligned}
$$

where

$$
\begin{aligned}
& F_{1,0}^{I I}=0, \quad G_{1,0}^{I I}=0, \quad F_{1,1}^{I I}=3 A_{1,0}^{\prime}+3 B_{1,0}^{\prime \prime}+f^{\prime}(Q) B_{1,0}, \quad G_{1,1}^{I I}=3 B_{1,0}^{\prime}, \\
& F_{2,0}^{I I}=-a_{1,0}\left(3 A_{1,0}^{\prime \prime}+f^{\prime}(Q) A_{1,0}\right)^{\prime}-\left(3 A_{1,0}^{\prime}+3 B_{1,0}^{\prime \prime}+f^{\prime}(Q) B_{1,0}\right) \delta_{m 2} \\
& G_{2,0}^{I I}=-\frac{1}{2} a_{1,0}\left(9 A_{1,0}^{\prime}+3 B_{1,0}^{\prime \prime}+f^{\prime}(Q) B_{1,0}\right)^{\prime}-\left(A_{1,0}+3 B_{1,0}^{\prime}\right) \delta_{m 2},
\end{aligned}
$$

and for $(k, l) \in \Sigma_{m}, F_{k, l}^{I I}, G_{k, l}^{I I}$ depend on $A_{k^{\prime}, l^{\prime}}, B_{k^{\prime}, l^{\prime}}$ such that $\left(k^{\prime}, l^{\prime}\right)<(k, l)$. Moreover, if $A_{k^{\prime}, l^{\prime}}$ are even and $B_{k^{\prime}, l^{\prime}}$ are odd then $F_{k, l}^{I I}$ are odd and $G_{k, l}^{I I}$ are even.

Finally, the following important property holds. Suppose (IP) holds for any $(k, l) \in \Sigma_{m}$ with $k+l \leq 2$. Then we have a sharp decomposition for each high order source term:

(1) For $m=2$,

$F_{3,0}^{I I I}=0, \quad G_{3,0}^{I I I}=-\frac{10}{3} A_{2,0}, \quad F_{2,1}^{I I I}=0, \quad G_{2,1}^{I I I}=-A_{1,1}+3 A_{2,0}, \quad F_{1,2}^{I I I}=G_{1,2}^{I I I}=0 \quad \bmod \mathcal{Y}$

(2) For $m=3$,

$$
F_{3,0}^{I I I}, G_{3,0}^{I I I}, F_{2,1}^{I I I} \in \mathcal{Y}, \quad G_{2,1}^{I I I}=3 A_{2,0}, \quad F_{4,0}^{I I I}=0, \quad G_{4,0}^{I I I}=-3 A_{2,0} \quad \bmod \mathcal{Y} .
$$

Proof. We have, thanks to the linearity of the operator $\mathbf{I I I}(\cdot)$,

$$
\mathbf{I I I}(W)=\sum_{(k, l) \in \Sigma_{m}} c^{l}\left(\mathbf{I I I}\left(A_{k, l}(y) Q_{c}^{k}\left(y_{c}\right)\right)+\mathbf{I I I}\left(B_{k, l}(y)\left(Q_{c}^{k}\right)^{\prime}\left(y_{c}\right)\right)\right) .
$$


In what follows, for commodity of notation we omit the variables $y, y_{c}$, if there is no related confusion. First, we compute $\mathbf{I I I}\left(A_{1,0}(y) Q_{c}\left(y_{c}\right)\right)$. By Claim 9 and the definition of $\beta$, we have

$$
\begin{aligned}
\mathbf{I I I}\left(A_{1,0} Q_{c}\right)= & -Q_{c}\left(\mathcal{L} A_{1,0}\right)^{\prime}+Q_{c}^{\prime}\left(3 A_{1,0}^{\prime \prime}+f^{\prime}(Q) A_{1,0}\right) \\
& +Q_{c}\left(-3 \beta A_{1,0}^{(3)}-\beta A_{1,0}^{\prime}-3 \beta_{x} A_{1,0}^{\prime \prime}-A_{1,0}^{\prime} \beta_{x x}+\beta A_{1,0}^{\prime}-\beta\left(f^{\prime}(Q) A_{1,0}\right)^{\prime}\right) \\
& +Q_{c}\left(3 \beta^{2} A_{1,0}^{(3)}+3 \beta \beta_{x} A_{1,0}^{\prime \prime}-\beta^{3} A_{1,0}^{(3)}+c \beta A_{1,0}^{\prime}\right) \\
& +Q_{c}^{\prime}\left(-c A_{1,0}-6 A_{1,0}^{\prime \prime} \beta-3 A_{1,0}^{\prime} \beta_{x}+3 A_{1,0}^{\prime \prime} \beta^{2}\right) \\
& +Q_{c}^{\prime \prime}\left(3 A_{1,0}^{\prime}-3 A_{1,0}^{\prime} \beta\right)+A_{1,0} Q_{c}^{(3)} \\
= & -\left(\mathcal{L} A_{1,0}\right)^{\prime} Q_{c}+\left(3 A_{1,0}^{\prime \prime}+f^{\prime}(Q) A_{1,0}\right) Q_{c}^{\prime}+3 A_{1,0}^{\prime \prime} c Q_{c} \\
& -\left(3 a_{1,0} A_{1,0}^{\prime \prime}+a_{1,0} f^{\prime}(Q) A_{1,0}+3 A_{1,0} \delta_{m 2}\right)^{\prime} Q_{c}^{2}-\left(\frac{9}{2} a_{1,0} A_{1,0}^{\prime \prime}+A_{1,0} \delta_{m 2}\right)\left(Q_{c}^{2}\right)^{\prime} \\
& +\sum_{3 \leq k+l \leq 4} c^{l}\left(F_{k, l} Q_{c}^{k}+G_{k, l}\left(Q_{c}^{k}\right)^{\prime}\right)+O\left(c Q_{c}^{3}+Q_{c}^{5}+c^{2} Q_{c}\right) .
\end{aligned}
$$

Moreover, by hypothesis $A_{1,0} \in \mathcal{Y}$ so we have all the source terms $F_{k, l}, G_{k, l} \in \mathcal{Y}$, as can be verified directly.

Now, we compute $\mathbf{I I I}\left(B_{1,0}(y) Q_{c}^{\prime}\left(y_{c}\right)\right)$ in a similar way:

$$
\begin{aligned}
\mathbf{I I I}\left(B_{1,0} Q_{c}^{\prime}\right)= & -Q_{c}^{\prime}\left(\mathcal{L} B_{1,0}\right)^{\prime}+Q_{c}^{\prime \prime}\left(3 B_{1,0}^{\prime \prime}+f^{\prime}(Q) B_{1,0}\right) \\
& +Q_{c}^{\prime}\left(-3 \beta B_{1,0}^{(3)}-\beta B_{1,0}^{\prime}-3 \beta_{x} B_{1,0}^{\prime \prime}-B_{1,0}^{\prime} \beta_{x x}+\beta B_{1,0}^{\prime}-\beta\left(f^{\prime}(Q) B_{1,0}\right)^{\prime}\right) \\
& +Q_{c}^{\prime}\left(3 \beta^{2} B_{1,0}^{(3)}+3 \beta \beta_{x} B_{1,0}^{\prime \prime}-\beta^{3} B_{1,0}^{(3)}+c \beta B_{1,0}^{\prime}\right) \\
& +Q_{c}^{\prime \prime}\left(-c B_{1,0}-6 B_{1,0}^{\prime \prime} \beta-3 B_{1,0}^{\prime} \beta_{x}+3 B_{1,0}^{\prime \prime} \beta^{2}\right) \\
& +Q_{c}^{(3)}\left(3 B_{1,0}^{\prime}-3 B_{1,0}^{\prime} \beta\right)+B_{1,0} Q_{c}^{(4)} \\
= & -\left(\mathcal{L} B_{1,0}\right)^{\prime} Q_{c}^{\prime}-Q_{c}^{2}\left(3 B_{1,0}^{\prime \prime}+f^{\prime}(Q) B_{1,0}\right) \delta_{m 2}+\left(3 B_{1,0}^{\prime \prime}+f^{\prime}(Q) B_{1,0}\right) c Q_{c} \\
& -\left(3 a_{1,0} A_{1,0}^{\prime \prime}+a_{1,0} f^{\prime}(Q) A_{1,0}+3 A_{1,0} \delta_{m 2}\right)^{\prime} Q_{c}^{2}-\left(\frac{9}{2} a_{1,0} A_{1,0}^{\prime \prime}+A_{1,0} \delta_{m 2}\right)\left(Q_{c}^{2}\right)^{\prime} \\
& +\sum_{3 \leq k+l \leq 4} c^{l}\left(F_{k, l} Q_{c}^{k}+G_{k, l}\left(Q_{c}^{k}\right)^{\prime}\right)+O\left(c Q_{c}^{3}+Q_{c}^{5}+c^{2} Q_{c}\right) .
\end{aligned}
$$

Suppose now that $2 \leq k+l \leq 4$. Here we will use (IP) for $k+l \leq 2$ to discard several terms of a tedious but direct computation. Indeed, from Claim 9 we have

$$
\begin{aligned}
\operatorname{III}\left(A_{k, l} Q_{c}^{k}\right)= & -Q_{c}^{k}\left(\mathcal{L} A_{k, l}\right)^{\prime}+\left(Q_{c}^{k}\right)^{\prime}\left(3 A_{k, l}^{\prime \prime}+f^{\prime}(Q) A_{k, l}\right) \\
+ & Q_{c}^{k}\left(-3 \beta A_{k, l}^{(3)}-\beta A_{k, l}^{\prime}-3 \beta_{x} A_{k, l}^{\prime \prime}-A_{k, l}^{\prime} \beta_{x x}+\beta A_{k, l}^{\prime}-\beta\left(f^{\prime}(Q) A_{k, l}\right)^{\prime}\right) \\
+ & Q_{c}^{k}\left(3 \beta^{2} A_{k, l}^{(3)}+3 \beta \beta_{x} A_{k, l}^{\prime \prime}-\beta^{3} A_{k, l}^{(3)}+c \beta A_{k, l}^{\prime}\right) \\
+ & \left(Q_{c}^{k}\right)^{\prime}\left(-c A_{k, l}-6 A_{k, l}^{\prime \prime} \beta-3 A_{k, l}^{\prime} \beta_{x}+3 A_{k, l}^{\prime \prime} \beta^{2}\right) \\
& +\left(Q_{c}^{k}\right)^{\prime \prime}\left(3 A_{k, l}^{\prime}-3 A_{k, l}^{\prime} \beta\right)+A_{k, l}\left(Q_{c}^{k}\right)^{(3)} \\
= & -Q_{c}^{k}\left(\mathcal{L} A_{k, l}\right)^{\prime}+\left(Q_{c}^{k}\right)^{\prime}\left(3 A_{k, l}^{\prime \prime}+f^{\prime}(Q) A_{k, l}\right)+A_{k, l}\left(Q_{c}^{k}\right)^{(3)}-A_{k, l} c\left(Q_{c}^{k}\right)^{\prime} \\
& +\sum_{\left(k^{\prime}, l^{\prime}\right) \in \Sigma_{m}^{\prime}} c^{l^{\prime}}\left(F_{k^{\prime}, l^{\prime}} Q_{c}^{k^{\prime}}+G_{k^{\prime}, l^{\prime}}\left(Q_{c}^{k^{\prime}}\right)^{\prime}\right)+O\left(c Q_{c}^{3}+Q_{c}^{5}+c^{2} Q_{c}\right) .
\end{aligned}
$$

Here $\Sigma_{m}^{\prime} \subseteq \Sigma_{m}$ is the set of indices of third order in $\Sigma_{m}$. More specificaly,

$$
\Sigma_{2}^{\prime}:=\{(1,2),(2,1),(3,0)\}, \quad \Sigma_{3}^{\prime}:=\{(2,1),(3,0),(4,0)\} .
$$

The terms describing $F_{k^{\prime}, l^{\prime}}$ and $G_{k^{\prime}, l^{\prime}}$ with $\left(k^{\prime}, l^{\prime}\right) \in \Sigma_{m}^{\prime}$ are in $\mathcal{Y}$ provided (IP) is satisfied for every $(k, l) \in \Sigma_{m} \backslash \Sigma_{m}^{\prime}$. 
Now note that from (129)

$$
\left(Q_{c}^{k}\right)^{(3)}=k^{2}\left(c Q_{c}^{k}\right)^{\prime}-\frac{k(2 k+m-1)}{m+1}\left(Q_{c}^{k+m-1}\right)^{\prime}-\varepsilon k \frac{k(2 k+p-1)}{p+1}\left(Q_{c}^{k+p-1}\right)^{\prime}+O\left(Q_{c}^{k+p}\right) .
$$

We can finally conclude that

$$
\begin{aligned}
\mathbf{I I I}\left(A_{k, l} Q_{c}^{k}\right)= & -Q_{c}^{k}\left(\mathcal{L} A_{k, l}\right)^{\prime}+\left(Q_{c}^{k}\right)^{\prime}\left(3 A_{k, l}^{\prime \prime}+f^{\prime}(Q) A_{k, l}\right)+\left(k^{2}-1\right) A_{k, l} c\left(Q_{c}^{k}\right)^{\prime} \\
& -\frac{k(2 k+m-1)}{m+1} A_{k, l}\left(Q_{c}^{k+m-1}\right)^{\prime}+\sum_{\left(k^{\prime}, l^{\prime}\right) \in \Sigma_{m}^{\prime}} c^{l^{\prime}}\left(F_{k^{\prime}, l^{\prime}} Q_{c}^{k^{\prime}}+G_{k^{\prime}, l^{\prime}}\left(Q_{c}^{k^{\prime}}\right)^{\prime}\right) \\
& +O\left(c Q_{c}^{3}+Q_{c}^{5}+c^{2} Q_{c}\right),
\end{aligned}
$$

where, as described above, the terms $F_{k^{\prime}, l^{\prime}}$ and $G_{k^{\prime}, l^{\prime}}$ with $\left(k^{\prime}, l^{\prime}\right) \in \Sigma_{m}^{\prime}$ are in $\mathcal{Y}$ provided (IP) is satisfied for every $(k, l) \in \Sigma_{m} \backslash \Sigma_{m}^{\prime}$.

On the other hand, the terms of the form

$$
\mathbf{I I I}\left(B_{k, l}\left(Q_{c}^{k}\right)^{\prime}\right), \quad(k, l) \in \Sigma_{m}, \quad 2 \leq k+l \leq 4,
$$

can be treated in the same way as above, and we only write the final result (see the computation of $\mathbf{I I I}\left(B_{1,0} Q_{c}^{\prime}\right)$ for example):

$$
\begin{aligned}
\operatorname{III}\left(B_{k, l}\left(Q_{c}^{k}\right)^{\prime}\right)= & -\left(Q_{c}^{k}\right)^{\prime}\left(\mathcal{L} B_{k, l}\right)^{\prime}+B_{k, l}\left(Q_{c}^{k}\right)^{(4)}-B_{k, l}\left(c Q_{c}^{k}\right)^{\prime \prime} \\
& +\sum_{1 \leq k^{\prime} \leq 4} c^{l^{\prime}}\left(F_{k^{\prime}, l^{\prime}} Q_{c}^{k^{\prime}}+G_{k^{\prime}, l^{\prime}}\left(Q_{c}^{k^{\prime}}\right)^{\prime}\right)+O\left(c Q_{c}^{3}+Q_{c}^{5}+c^{2} Q_{c}\right) \\
= & -\left(Q_{c}^{k}\right)^{\prime}\left(\mathcal{L} B_{k, l}\right)^{\prime}+\sum_{\substack{\left(k^{\prime}, l^{\prime}\right) \in \Sigma_{m}^{\prime} \\
(k, l) \leq\left(k^{\prime}, l^{\prime}\right)}} c^{l^{\prime}}\left(F_{k^{\prime}, l^{\prime}} Q_{c}^{k^{\prime}}+G_{k^{\prime}, l^{\prime}}\left(Q_{c}^{k^{\prime}}\right)^{\prime}\right)+O\left(c Q_{c}^{3}+Q_{c}^{5}+c^{2} Q_{c}\right) .
\end{aligned}
$$

To obtain (135) and (136) we only evaluate the expressions for $\mathbf{I I I}\left(A_{k, l} Q_{c}^{k}\right)$ and $\mathbf{I I I}\left(B_{k, l}\left(Q_{c}^{k}\right)^{\prime}\right)$ for each $(k, l) \in \Sigma_{m}$ with $2 \leq k+l$. The final result follows from the sum of each term $\mathbf{I I I}\left(A_{k, l} Q_{c}^{k}\right)$, $\operatorname{III}\left(B_{k, l}\left(Q_{c}^{k}\right)^{\prime}\right)$ for $(k, l) \in \Sigma_{m}$, discarding localized terms. This concludes the proof.

The final term reads

Lemma A.6.

$$
\mathbf{I V}=\sum_{(k, l) \in \Sigma_{m}} c^{l}\left(Q_{c}^{k}\left(y_{c}\right) F_{k, l}^{I V}(y)+\left(Q_{c}^{k}\right)^{\prime}\left(y_{c}\right) G_{k, l}^{I V}(y)\right)+c^{3} O\left(Q_{c}\right)
$$

where

$$
\begin{aligned}
& F_{1,0}^{I V}=G_{1,0}^{I V}=0, \quad F_{1,1}^{I V}=G_{1,1}^{I V}=0 \\
& F_{2,0}^{I V}=\frac{1}{2}\left(f^{\prime \prime}(Q)\left(2 A_{1,0}+A_{1,0}^{2}\right)\right)^{\prime}, \quad G_{2,0}^{I V}=\frac{1}{2}\left[f^{\prime \prime}(Q)\left(2 A_{1,0}+A_{1,0}^{2}\right)+\left(f^{\prime \prime}(Q)\left(B_{1,0}+A_{1,0} B_{1,0}\right)\right)^{\prime}\right]
\end{aligned}
$$

and for $(k, l) \in \Sigma_{m}^{\prime}$ (see 137)), $F_{k, l}^{I V}, G_{k, l}^{I V}$ depend on $A_{k^{\prime}, l^{\prime}}, B_{k^{\prime}, l^{\prime}}$ for $\left(k^{\prime}, l^{\prime}\right) \in \Sigma_{m}$ with $\left(k^{\prime}, l^{\prime}\right)<$ $(k, l)$. Moreover, if $A_{k^{\prime}, l^{\prime}}$ are even and $B_{k^{\prime}, l^{\prime}}$ are odd then $F_{k, l}^{I V}$ are odd and $G_{k, l}^{I V}$ are even.

Finally, suppose (IP) holds for $(k, l) \in \Sigma_{m}$ with $k+l \leq 2$. Then the only non localized terms for $(k, l) \in \Sigma_{m}^{\prime}$ are given by

$$
F_{2,1}^{I V}=0, \quad G_{2,1}^{I V}=\frac{1}{2} f^{\prime \prime}(Q)\left(2 A_{1,1}+B_{1,0}^{2}\right) \quad \bmod \mathcal{Y}
$$

and

$$
F_{3,0}^{I V}=0, \quad G_{3,0}^{I V}=f^{\prime \prime}(Q)\left(A_{2,0}-\frac{1}{3} B_{1,0}^{2}\right) \quad \bmod \mathcal{Y}
$$

for the quadratic case, and

$$
G_{4,0}^{I V}=\frac{1}{2} f^{(3)}(Q) A_{2,0} \quad \bmod \mathcal{Y}
$$

in the cubic case. 
Proof. As above, first define $\mathbf{I} \tilde{\mathbf{V}}:=f\left(R+R_{c}+W\right)-f\left(R+R_{c}\right)-f^{\prime}(R) W$. Note that, using that $R:=Q(y)$ and $R_{c}:=Q_{c}\left(y_{c}\right)$,

$$
\begin{aligned}
\tilde{\mathbf{V}}= & \left(f^{\prime}\left(Q+Q_{c}\right)-f^{\prime}(Q)\right) W+\frac{1}{2} f^{\prime \prime}\left(Q+Q_{c}\right) W^{2}+\frac{1}{6} f^{(3)}\left(Q+Q_{c}\right) W^{3} \\
& +\frac{1}{24} f^{(4)}\left(Q+Q_{c}\right) W^{4}+O\left(W^{5}\right) \\
= & {\left[f^{\prime \prime}(Q) Q_{c}+\frac{1}{2} f^{(3)}(Q) Q_{c}^{2}+\frac{1}{6} f^{(4)}(Q) Q_{c}^{3}+O\left(Q_{c}^{4}\right)\right] W } \\
& +\frac{1}{2}\left[f^{\prime \prime}(Q)+f^{(3)}(Q) Q_{c}+\frac{1}{2} f^{(4)}(Q) Q_{c}^{2}+O\left(Q_{c}\right)^{3}\right] W^{2} \\
& +\frac{1}{6}\left[f^{(3)}(Q)+f^{(4)}(Q) Q_{c}+O\left(Q_{c}^{2}\right)\right] W^{3}+\frac{1}{24} f^{(4)}(Q) W^{4}+O\left(Q_{c}^{5}\right) \\
= & f^{\prime \prime}(Q)\left(Q_{c} W+\frac{1}{2} W^{2}\right)+\frac{1}{2} f^{(3)}(Q)\left(Q_{c}^{2} W+W^{2} Q_{c}+\frac{1}{3} W^{3}\right) \\
& +\frac{1}{2} f^{(4)}(Q)\left(\frac{1}{3} Q_{c}^{3} W+\frac{1}{2} Q_{c}^{2} W^{2}+\frac{1}{3} Q_{c} W^{3}+\frac{1}{12} W^{4}\right)+O\left(Q_{c}^{5}\right) .
\end{aligned}
$$

Now, the final value of $\mathbf{I V}$ depends on the different values of $m$. We will proceed carefully in both cases.

Case $m=2$. Here we consider only up to third order, namely

$$
\begin{aligned}
\tilde{\mathbf{I}} & =\frac{1}{2} f^{\prime \prime}(Q)\left(2 Q_{c} W+W^{2}\right)+\frac{1}{2} f^{(3)}(Q)\left(Q_{c}^{2} W+W^{2} Q_{c}+\frac{1}{3} W^{3}\right)+O\left(Q_{c}^{4}\right) \\
& =: \quad \tilde{\mathbf{I}}_{2}+\mathbf{I} \tilde{\mathbf{V}}_{3}+O\left(Q_{c}^{4}\right) .
\end{aligned}
$$

First of all let us consider the third order term $\mathbf{I} \tilde{\mathbf{V}}_{3}$. A quickly computation using (33) gives us

$$
Q_{c}^{2} W=A_{1,0} Q_{c}^{3}+\frac{1}{3} B_{1,0}\left(Q_{c}^{3}\right)^{\prime}+O\left(c^{3} Q_{c}\right)
$$

and

$$
W^{2} Q_{c}=A_{1,0}^{2} Q_{c}^{3}+\frac{2}{3} A_{1,0} B_{1,0}\left(Q_{c}^{3}\right)^{\prime}+O\left(c^{3} Q_{c}\right), \quad W^{3}=A_{1,0}^{3} Q_{c}^{3}+A_{1,0} B_{1,0}\left(Q_{c}^{3}\right)^{\prime}+O\left(c^{3} Q_{c}\right) .
$$

If we suppose $A_{1,0} \in \mathcal{Y}$ and $B_{1,0}$ bounded (this is actually the case), we will obtain

$$
\mathbf{I} \tilde{\mathbf{V}}_{3}=F_{3,0}^{I \tilde{V}_{3}} Q_{c}^{3}+\left(G_{3,0}^{I \tilde{V}_{3}}+\frac{1}{6} f^{(3)}(Q) B_{1,0}\right)\left(Q_{c}^{3}\right)^{\prime}+O\left(c^{3} Q_{c}\right)
$$

where $F_{3,0}^{I \tilde{V}_{3}}, G_{3,0}^{I \tilde{V}{ }_{3}} \in \mathcal{Y}$. Moreover, for $p \geq 4$, actually $f^{(3)}(Q)-6 \mu(\varepsilon) \in \mathcal{Y}$, because of

$$
f^{(3)}(Q)=6 \mu(\varepsilon)+p(p-1)(p-2) \varepsilon Q^{p-3}+f_{1}^{(3)}(Q) .
$$

Now, let us compute in detail the term $\mathbf{I} \tilde{\mathbf{V}}_{2}$. These terms above are important because they will give us source terms of second order. Now from the definition of $W$ in (33) it is easy to check that, up to third order,

$$
Q_{c} W=A_{1,0} Q_{c}^{2}+\frac{1}{2} B_{1,0}\left(Q_{c}^{2}\right)^{\prime}+A_{1,1} c Q_{c}^{2}+\frac{1}{2} B_{1,1} c\left(Q_{c}^{2}\right)^{\prime}+A_{2,0} Q_{c}^{3}+\frac{2}{3} B_{2,0}\left(Q_{c}^{3}\right)^{\prime}+O\left(c^{3} Q_{c}\right),
$$

and

$$
\begin{aligned}
W^{2}= & A_{1,0}^{2} Q_{c}^{2}+A_{1,0} B_{1,0}\left(Q_{c}^{2}\right)^{\prime}+\left(2 A_{1,0} A_{1,1}+B_{1,0}^{2}\right) c Q_{c}^{2}+\left(A_{1,0} B_{1,1}+B_{1,0} A_{1,1}\right) c\left(Q_{c}^{2}\right)^{\prime} \\
& +\left(2 A_{1,0} A_{2,0}-\frac{2}{3} B_{1,0}^{2}\right) Q_{c}^{3}+\frac{2}{3}\left(A_{1,0} B_{2,0}+B_{1,0} A_{2,0}\right)\left(Q_{c}^{3}\right)^{\prime} .
\end{aligned}
$$


From here,

$$
\begin{aligned}
\tilde{\mathbf{V}}_{\mathbf{2}}= & \frac{1}{2} f^{\prime \prime}(Q)\left(2 A_{1,0}+A_{1,0}^{2}\right) Q_{c}^{2}+\frac{1}{2} f^{\prime \prime}(Q)\left(B_{1,0}+A_{1,0} B_{1,0}\right)\left(Q_{c}^{2}\right)^{\prime} \\
& +\frac{1}{2} f^{\prime \prime}(Q)\left(F_{2,1}^{I \tilde{V}_{2}}+2 A_{1,1}+B_{1,0}^{2}\right) c Q_{c}^{2}+\frac{1}{2} f^{\prime \prime}(Q)\left(G_{2,1}^{I \tilde{V}_{2}}+B_{1,1}+A_{1,1} B_{1,0}\right) c\left(Q_{c}^{2}\right)^{\prime} \\
& +\frac{1}{2} f^{\prime \prime}(Q)\left(F_{3,0}^{I \tilde{V}_{2}}+2 A_{2,0}-\frac{2}{3} B_{1,0}^{2}\right) Q_{c}^{3}+\frac{1}{2} f^{\prime \prime}(Q)\left(G_{3,0}^{I \tilde{V}_{2}}+\frac{4}{3} B_{2,0}+\frac{2}{3} A_{2,0} B_{1,0}\right)\left(Q_{c}^{3}\right)^{\prime} \\
& +O\left(c^{3} Q_{c}\right),
\end{aligned}
$$

where for $k+l=3$ it is satisfied $F_{k, l}^{I \tilde{V}}, G_{k, l}^{I \tilde{V}_{2}} \in \mathcal{Y}$, provided $A_{1,0} \in \mathcal{Y}$ and $B_{1,0}$ is bounded (namely $(k, l)=(1,0)$ satisfies $(\mathbf{I P}))$.

Putting all this information together, and after derivation, we obtain (note that $p \geq 3$ and $\left.\left(f^{\prime \prime}(Q)\right)^{\prime} \in \mathcal{Y}\right)$

$$
\begin{aligned}
\mathbf{I V}= & \left(\tilde{\mathbf{I V}}_{2}+\mathbf{I}_{3}+O\left(Q_{c}^{4}\right)\right)_{x} \\
= & \frac{1}{2}\left(f^{\prime \prime}(Q)\right)^{\prime}(1-\beta)\left(2 A_{1,0}+A_{1,0}^{2}\right) Q_{c}^{2}+\frac{1}{2} f^{\prime \prime}(Q)\left(2 A_{1,0}+A_{1,0}^{2}\right)^{\prime}(1-\beta) Q_{c}^{2} \\
& +\frac{1}{2} f^{\prime \prime}(Q)\left(2 A_{1,0}+A_{1,0}^{2}\right)\left(Q_{c}^{2}\right)^{\prime}+\frac{1}{2}\left(f^{\prime \prime}(Q)\right)^{\prime}(1-\beta)\left(B_{1,0}+A_{1,0} B_{1,0}\right)\left(Q_{c}^{2}\right)^{\prime} \\
& +\frac{1}{2} f^{\prime \prime}(Q)(1-\beta)\left(B_{1,0}+A_{1,0} B_{1,0}\right)^{\prime}\left(Q_{c}^{2}\right)^{\prime} \\
& +F_{2,1}^{I V} c Q_{c}^{2}+\left[G_{2,1}^{I V}+\frac{1}{2} f^{\prime \prime}(Q)\left(2 A_{1,1}+B_{1,0}^{2}\right)\right]\left(c Q_{c}^{2}\right)^{\prime} \\
& +\left[F_{3,0}^{I V}+f^{\prime \prime}(Q)\left(A_{2,0}-\frac{1}{3} B_{1,0}^{2}\right)\right]\left(Q_{c}^{3}\right)^{\prime}+\left[G_{3,0}^{I V}+f^{\prime \prime}(Q)\left(\frac{2}{3} B_{2,0}+\frac{1}{3} A_{2,0} B_{1,0}\right)\right]\left(Q_{c}^{3}\right)^{\prime \prime} \\
& +O\left(c^{3} Q_{c}\right), \\
& \frac{1}{2}\left[f^{\prime \prime}(Q)\left(2 A_{1,0}+A_{1,0}^{2}\right)\right]^{\prime} Q_{c}^{2} \\
& +\frac{1}{2}\left[f^{\prime \prime}(Q)\left(2 A_{1,0}+A_{1,0}^{2}\right)+\left(f^{\prime \prime}(Q)\left(B_{1,0}+A_{1,0} B_{1,0}\right)\right)^{\prime}\right]\left(Q_{c}^{2}\right)^{\prime} \\
& +F_{2,1}^{I V} c Q_{c}^{2}+\left[G_{2,1}^{I V}+\frac{1}{2} f^{\prime \prime}(Q)\left(2 A_{1,1}+B_{1,0}^{2}\right)\right]\left(c Q_{c}^{2}\right)^{\prime}+F_{3,0}^{I V} Q_{c}^{3} \\
& +\left[G_{3,0}^{I V}+f^{\prime \prime}(Q)\left(A_{2,0}-\frac{1}{3} B_{1,0}^{2}\right)\right]\left(Q_{c}^{3}\right)^{\prime}+O\left(c^{3} Q_{c}\right),
\end{aligned}
$$

where $F_{2,1}^{I V}, G_{2,1}^{I V}, F_{3,0}^{I V}$ and $G_{3,0}^{I V}$ are $\mathcal{Y}$-functions provided property (IP) holds for $k+l \leq 2$. We finally get the Lemma in the quadratic case, the decomposition (139), (140) and (141), with the desired properties.

Case $m=3$. Here we consider up to fourth order in our computations. First of all, we write

$$
\begin{aligned}
\tilde{\mathbf{I}}= & \frac{1}{2} f^{\prime \prime}(Q)\left(2 Q_{c} W+W^{2}\right)+\frac{1}{2} f^{(3)}(Q)\left(Q_{c}^{2} W+W^{2} Q_{c}+\frac{1}{3} W^{3}\right) \\
& +\frac{1}{2} f^{(4)}(Q)\left(\frac{1}{3} Q_{c}^{3} W+\frac{1}{2} Q_{c}^{2} W^{2}+\frac{1}{3} Q_{c} W^{3}+\frac{1}{12} W^{4}\right)+O\left(Q_{c}^{5}\right) \\
=: & \tilde{\mathbf{V}}_{\mathbf{2}}+\tilde{\mathbf{I V}}_{\mathbf{3}}+\tilde{\mathbf{I V}}_{\mathbf{4}}+O\left(Q_{c}^{5}\right) .
\end{aligned}
$$

From now on, and for the sake of simplicity in our computations, we will consider that property (IP) holds for any $(k, l) \in \Sigma_{3}$ and that $A_{1,0} \in \mathcal{Y}$. We recall that in the cubic case, our correction term is given by

$$
\begin{aligned}
W= & A_{1,0} Q_{c}+B_{1,0} Q_{c}^{\prime}+A_{1,1} c Q_{c}+B_{1,1} c Q_{c}^{\prime} \\
& +A_{2,0} Q_{c}^{2}+B_{2,0}\left(Q_{c}^{2}\right)^{\prime}+A_{3,0} Q_{c}^{3}+B_{3,0}\left(Q_{c}^{3}\right)^{\prime} \\
& +A_{2,1} c Q_{c}^{2}+B_{2,1} c\left(Q_{c}^{2}\right)^{\prime}+A_{4,0} Q_{c}^{4}+B_{4,0}\left(Q_{c}^{4}\right)^{\prime}
\end{aligned}
$$


Let us first consider the term $\mathbf{I} \tilde{\mathbf{V}}_{\mathbf{2}}$. Note that

$$
\begin{aligned}
Q_{c} W= & A_{1,0} Q_{c}^{2}+\frac{1}{2} B_{1,0}\left(Q_{c}^{2}\right)^{\prime}+A_{1,1} c Q_{c}^{2}+\frac{1}{2} B_{1,1} c\left(Q_{c}^{2}\right)^{\prime}+A_{2,0} Q_{c}^{3}+\frac{2}{3} B_{2,0}\left(Q_{c}^{3}\right)^{\prime} \\
& +A_{3,0} Q_{c}^{4}+\frac{3}{4} B_{3,0}\left(Q_{c}^{4}\right)^{\prime}+O\left(c Q_{c}^{3}+Q_{c}^{5}\right) .
\end{aligned}
$$

Using (128) we get

$$
\begin{aligned}
W^{2}= & A_{1,0}^{2} Q_{c}^{2}+B_{1,0}^{2} Q_{c}^{\prime 2}+A_{2,0}^{4} Q_{c}^{4} \\
& +A_{1,0} B_{1,0}\left(Q_{c}^{2}\right)^{\prime}+2 A_{1,0} A_{1,1} c Q_{c}^{2}+A_{1,0} B_{1,1} c\left(Q_{c}^{2}\right)^{\prime} \\
& +2 A_{1,0} A_{2,0} Q_{c}^{3}+\frac{4}{3} A_{1,0} B_{2,0}\left(Q_{c}^{3}\right)^{\prime}+2 A_{1,0} A_{3,0} Q_{c}^{4}+\frac{3}{2} A_{1,0} B_{3,0}\left(Q_{c}^{4}\right)^{\prime} \\
& +A_{1,1} B_{1,0} c\left(Q_{c}^{2}\right)^{\prime}+\frac{2}{3} A_{2,0} B_{1,0}\left(Q_{c}^{3}\right)^{\prime}+\frac{1}{2} A_{3,0} B_{1,0}\left(Q_{c}^{4}\right)^{\prime}+O\left(c Q_{c}^{3}+Q_{c}^{5}\right) \\
= & A_{1,0}^{2} Q_{c}^{2}+A_{1,0} B_{1,0}\left(Q_{c}^{2}\right)^{\prime}+\left(2 A_{1,0} A_{1,1}+B_{1,0}^{2}\right) c Q_{c}^{2} \\
& +\left(A_{1,1} B_{1,0}+A_{1,0} B_{1,1}\right) c\left(Q_{c}^{2}\right)^{\prime}+2 A_{1,0} A_{2,0} Q_{c}^{3}+\frac{2}{3}\left(2 A_{1,0} B_{2,0}+A_{2,0} B_{1,0}\right)\left(Q_{c}^{3}\right)^{\prime} \\
& +\left(2 A_{1,0} A_{3,0}+A_{2,0}^{4}-\frac{1}{2} B_{1,0}^{2}\right) Q_{c}^{4}+\frac{1}{2}\left(3 A_{1,0} B_{3,0}+A_{3,0} B_{1,0}\right)\left(Q_{c}^{4}\right)^{\prime}+O\left(c Q_{c}^{3}+Q_{c}^{5}\right) .
\end{aligned}
$$

From here, and using the (IP) property, we get (note that $f^{\prime \prime}(Q) \in \mathcal{Y}$ )

$$
\begin{aligned}
\tilde{\mathbf{I}}_{\mathbf{2}}= & \frac{1}{2} f^{\prime \prime}(Q)\left(2 A_{1,0}+A_{1,0}^{2}\right) Q_{c}^{2}+\frac{1}{2} f^{\prime \prime}(Q)\left(B_{1,0}+A_{1,0} B_{1,0}\right)\left(Q_{c}^{2}\right)^{\prime} \\
& +\sum_{(k, l) \in \Sigma_{3}^{\prime}} c^{l}\left(F_{k, l}^{I \tilde{V}_{2}} Q_{c}^{k}+G_{k, l}^{I \tilde{V}_{2}}\left(Q_{c}^{k}\right)^{\prime}\right)+O\left(c Q_{c}^{3}+Q_{c}^{5}\right),
\end{aligned}
$$

where $\Sigma_{3}^{\prime}$ was introduced in (137), and $F_{k, l}^{I \tilde{V}}, G_{k, l}^{I \tilde{V}{ }_{2}} \in \mathcal{Y}$.

Now we deal with $\mathbf{I V}_{\mathbf{3}}$. Here we have

$$
Q_{c}^{2} W=A_{1,0} Q_{c}^{3}+\frac{1}{3} B_{1,0}\left(Q_{c}^{3}\right)^{\prime}+A_{2,0} Q_{c}^{4}+\frac{1}{4} B_{2,0}\left(Q_{c}^{4}\right)^{\prime}+O\left(c Q_{c}^{3}+Q_{c}^{5}\right) .
$$

and

$$
\begin{aligned}
Q_{c} W^{2}= & A_{1,0}^{2} Q_{c}^{3}+\frac{2}{3} A_{1,0} B_{1,0}\left(Q_{c}^{3}\right)^{\prime}+2 A_{1,0} A_{2,0} Q_{c}^{4} \\
& +\frac{1}{2}\left(2 A_{1,0} B_{2,0}+A_{2,0} B_{1,0}\right)\left(Q_{c}^{4}\right)^{\prime}+O\left(c Q_{c}^{3}+Q_{c}^{5}\right) .
\end{aligned}
$$

Finally

$$
\begin{aligned}
W^{3}= & W^{2} W \\
= & A_{1,0}^{3} Q_{c}^{3}+\frac{2}{3} A_{1,0}^{2} B_{1,0}\left(Q_{c}^{3}\right)^{\prime}+2 A_{1,0}^{2} A_{2,0} Q_{c}^{4}+\frac{1}{2}\left(2 A_{1,0}^{2} B_{2,0}+A_{1,0} A_{2,0} B_{1,0}\right)\left(Q_{c}^{4}\right)^{\prime} \\
& +\frac{1}{3} B_{1,0} A_{1,0}^{2}\left(Q_{c}^{3}\right)^{\prime}+\frac{1}{2} A_{1,0} B_{1,0} A_{2,0}\left(Q_{c}^{4}\right)^{\prime} \\
& +A_{2,0} A_{1,0}^{2} Q_{c}^{4}+\frac{1}{2} A_{2,0} A_{1,0} B_{1,0}\left(Q_{c}^{4}\right)^{\prime}+\frac{1}{2} A_{1,0}^{2} B_{2,0}\left(Q_{c}^{4}\right)^{\prime}+O\left(c Q_{c}^{3}+Q_{c}^{5}\right) \\
= & A_{1,0}^{3} Q_{c}^{3}+A_{1,0}^{2} B_{1,0}\left(Q_{c}^{3}\right)^{\prime}+3 A_{1,0}^{2} A_{2,0} Q_{c}^{4}+\frac{3}{2}\left(A_{1,0}^{2} B_{2,0}+A_{1,0} A_{2,0} B_{1,0}\right)\left(Q_{c}^{4}\right)^{\prime} \\
& +O\left(c Q_{c}^{3}+Q_{c}^{5}\right) .
\end{aligned}
$$

From here, and using the (IP) property, we get

$$
\begin{aligned}
\tilde{\mathbf{V}}_{\mathbf{3}}= & \frac{1}{2} f^{(3)}(Q)\left[\frac{1}{3} B_{1,0}\left(Q_{c}^{3}\right)^{\prime}+A_{2,0} Q_{c}^{4}+\frac{1}{4}\left(2 A_{2,0} B_{1,0}+B_{2,0}\right)\left(Q_{c}^{4}\right)^{\prime}\right] \\
& +\sum_{(k, l) \in \Sigma_{3}^{\prime}} c^{l}\left(F_{k, l}^{I \tilde{V}_{2}} Q_{c}^{k}+G_{k, l}^{I \tilde{V}_{2}}\left(Q_{c}^{k}\right)^{\prime}\right)+O\left(c Q_{c}^{3}+Q_{c}^{5}\right),
\end{aligned}
$$

where $\Sigma_{3}^{\prime}$ was introduced in (137), and $F_{k, l}^{I \tilde{V} V_{3}}, G_{k, l}^{I V_{3}} \in \mathcal{Y}$. 
Finally, fourth order terms are easy to compute:

and

$$
\begin{gathered}
Q_{c}^{3} W=A_{1,0} Q_{c}^{4}+\frac{1}{4} B_{1,0}\left(Q_{c}^{4}\right)^{\prime}+O\left(Q_{c}^{5}+c Q_{c}^{3}\right), \\
Q_{c}^{2} W^{2}=A_{1,0}^{2} Q_{c}^{4}+\frac{1}{2} A_{1,0} B_{1,0}\left(Q_{c}^{4}\right)^{\prime}+O\left(Q_{c}^{5}+c Q_{c}^{3}\right), \\
Q_{c} W^{3}=A_{1,0}^{3} Q_{c}^{4}+\frac{3}{4} A_{1,0}^{2} B_{1,0}\left(Q_{c}^{4}\right)^{\prime}+O\left(Q_{c}^{5}+c Q_{c}^{3}\right),
\end{gathered}
$$

$$
W^{4}=A_{1,0}^{4} Q_{c}^{4}+A_{1,0}^{3} B_{1,0}\left(Q_{c}^{4}\right)^{\prime}+O\left(Q_{c}^{5}+c Q_{c}^{3}\right) .
$$

As we have supposed $A_{1,0} \in \mathcal{Y}((1,0)$ satisfies (IP)), we will obtain

$$
\tilde{\mathbf{I}}_{\mathbf{4}}=F_{4,0}^{I \tilde{V}_{4}} Q_{c}^{4}+\left[G_{4,0}^{I \tilde{V}_{4}}+\frac{1}{24} f^{(4)}(Q) B_{1,0}\right]\left(Q_{c}^{4}\right)^{\prime}+O\left(Q_{c}^{5}+c Q_{c}^{3}\right)
$$

where $F_{4,0}^{I \tilde{V}_{4}}, G_{4,0}^{I \tilde{V}_{4}} \in \mathcal{Y}$.

We finally collect the expansions of $\mathbf{I V}_{\mathbf{2}}, \mathbf{I} \tilde{\mathbf{V}}_{\mathbf{3}}$ and $\mathbf{I} \tilde{\mathbf{V}}_{\mathbf{4}}$. We derivate to obtain

$$
\begin{aligned}
\mathbf{I V}= & \left(\tilde{\mathbf{I}}_{\mathbf{2}}+\mathbf{I} \tilde{\mathbf{V}}_{\mathbf{3}}+\mathbf{I}_{\mathbf{4}}+O\left(Q_{c}^{5}\right)\right)_{x} \\
& \frac{1}{2}\left(f^{\prime \prime}(Q)\left(2 A_{1,0}+A_{1,0}^{2}\right)\right)^{\prime} Q_{c}^{2}+\frac{1}{2} f^{(3)}(Q) A_{2,0}\left(Q_{c}^{4}\right)^{\prime} \\
& +\frac{1}{2}\left[f^{\prime \prime}(Q)\left(2 A_{1,0}+A_{1,0}^{2}\right)+\left(f^{\prime \prime}(Q)\left(B_{1,0}+A_{1,0} B_{1,0}\right)\right)^{\prime}\right]\left(Q_{c}^{2}\right)^{\prime} \\
& +\sum_{(k, l) \in \Sigma_{3}^{\prime}} c^{l}\left(F_{k, l}^{I \tilde{V}} Q_{c}^{k}+G_{k, l}^{I V}\left(Q_{c}^{k}\right)^{\prime}\right)+O\left(c Q_{c}^{3}+Q_{c}^{5}\right),
\end{aligned}
$$

where, as we have emphasized, $F_{k, l}^{I \tilde{V}}, G_{k, l}^{I \tilde{V}} \in \mathcal{Y}$ provided $A_{k^{\prime}, l^{\prime}}, B_{k^{\prime}, l^{\prime}}$ satisfy the (IP) property for $\left(k^{\prime}, l^{\prime}\right)<(k, l)$, as is the case. Here we have also used that $\left(f^{\prime \prime}(Q)\right)^{\prime},\left(f^{(4)}(Q)\right)^{\prime} \in \mathcal{Y}$ for all $p \geq 4$. The set $\Sigma_{3}^{\prime}$ was defined in (137).

Let us finally prove (v). From (i), the rest term $\mathcal{E}(t, x)$ is a finite sum of terms of the type $c^{l} Q_{c}^{k}\left(y_{c}\right) f(y)$ or $c^{l}\left(Q_{c}^{k}\right)^{\prime}\left(y_{c}\right) f(y)$, where $(k, l) \notin \Sigma_{m}$. More specifically, this means $k+l \geq 4$ for $m=2$ and $(k, l)=(1,2),(3,1)$ or higher order terms (excluding $(k, l)=(4,0))$ in the case $m=3$ (see the definition of $\Sigma_{m}$ in Section 2.1). Here $f$ is a bounded function such that $f^{\prime} \in \mathcal{Y}$. Thus, we easily conclude, using Claim 4

$$
\|\mathcal{E}(t)\|_{H^{1}(\mathbb{R})} \leq K c^{3\left(\frac{1}{m-1}+\frac{1}{4}\right)},
$$

as desired. This finishes the proof.

Putting together Lemmas A.3 A.6, we obtain Proposition A.1, in particular, the explicit expressions of $F_{k, l}$ and $G_{k, l}$ for $1 \leq k+l \leq 2$.

\section{Appendix B. End of proof of Proposition 2.6}

Continuing with the proof of Proposition 2.6. we show now the existence of a nonzero residual term appearing after the collision.

B.1. General computations. We proceed to compute the constants $b_{2,0}$ more explicitly. In the course of the proof we will made use several times of the equations satisfied by the functions $A_{k, l}, B_{k, l}, a_{k, l}$ for $(k, l)=(1,0)$ and $(2,0)$, cf. (45)-(46) for the system $\left(\Omega_{1,0}\right)$ and (50)-(51) for the second one.

Claim 11 (Explicit value of $b_{2,0}$ ). Suppose $f$ as in (10). Then the following expressions for the $b_{2,0}$ coefficient hold. 
(1) Case $m=2$.

$$
\begin{aligned}
b_{2,0}=- & \frac{1}{2} b_{1,0}^{3}+\frac{1}{4} \int_{\mathbb{R}}\left(f^{\prime \prime}(Q)-2\right)\left(1+A_{1,0}\right)^{3}-2 b_{1,0}+\frac{1}{2} \int_{\mathbb{R}} A_{1,0}\left(1+A_{1,0}^{2}\right) \\
& -\frac{1}{2} a_{1,0} \int_{\mathbb{R}} Q A_{1,0}-\frac{3}{4} a_{1,0}^{3} \int_{\mathbb{R}} Q^{\prime 2}+\frac{1}{2} a_{1,0}^{2} \int_{\mathbb{R}} Q\left[Q-f^{\prime}(Q)\left(1+A_{1,0}\right)\right] \\
& -\frac{3}{4} a_{1,0} \int_{\mathbb{R}}\left[f^{\prime}(Q)\left(1+A_{1,0}\right)+3 A_{1,0}^{\prime \prime}\right] A_{1,0}+\frac{1}{2} \int_{\mathbb{R}} B_{1,0}\left[3 A_{1,0}^{\prime}+f^{\prime}(Q) \int_{0}^{x}\left(A_{1,0}+a_{1,0} Q\right)\right] \\
& +3 a_{1,0}^{2} \int_{\mathbb{R}} Q^{\prime \prime} A_{1,0} .
\end{aligned}
$$

(2) Case $m=3$.

$$
\begin{aligned}
b_{2,0}= & \frac{1}{4} \int_{\mathbb{R}} f^{\prime \prime}(Q)\left(1+A_{1,0}\right)^{3}-\frac{3}{4} a_{1,0} \int_{\mathbb{R}} f^{\prime}(Q)\left(1+A_{1,0}\right) A_{1,0}+\frac{9}{4} a_{1,0} \int_{\mathbb{R}} A_{1,0}^{\prime 2} \\
& -\frac{1}{2} a_{1,0}^{2} \int_{\mathbb{R}} f^{\prime}(Q) Q\left(1+A_{1,0}\right)+3 a_{1,0}^{2} \int_{\mathbb{R}} A_{1,0} Q^{\prime \prime}-\frac{3}{4} a_{1,0}^{3} \int_{\mathbb{R}} Q^{\prime 2} .
\end{aligned}
$$

Proof. We treat first the cubic case, being easier. Let us start with (58) and (59). In this case, we have a priori chosen $A_{2,0} \in \mathcal{Y}$, so that $\gamma_{2,0}=0$, and then from (47)

$$
b_{2,0}=\frac{1}{2}\left[-a_{1,0} \int_{\mathbb{R}} G_{2,0} Q-a_{1,0} \int_{\mathbb{R}} \tilde{F}_{2,0} P+\int_{\mathbb{R}} \tilde{F}_{2,0} \bar{P}+\int_{\mathbb{R}} G_{2,0}\right],
$$

where $\tilde{F}_{2,0}^{\prime}=F_{2,0}, \tilde{F}_{2,0} \in \mathcal{Y}$. More precisely,

$$
\tilde{F}_{2,0}:=\frac{1}{2} f^{\prime \prime}(Q)\left(1+A_{1,0}\right)^{2}+3 a_{1,0}^{2} Q^{\prime \prime}-a_{1,0}\left(3 A_{1,0}^{\prime \prime}+f^{\prime}(Q)\left(1+A_{1,0}\right)\right) .
$$

First, it is easy to see from (55) by using the (IP) property for $(k, l)=(1,0)$, that

$$
\int_{\mathbb{R}} G_{2,0}=\frac{1}{2} \int_{\mathbb{R}} f^{\prime \prime}(Q)\left(1+A_{1,0}\right)^{2} .
$$

Secondly, from (48), (38) and (42), $\bar{P}-a_{1,0} P=A_{1,0}+a_{1,0} Q$, and thus

$$
b_{2,0}=\frac{1}{2}\left[a_{1,0} \int_{\mathbb{R}}\left(\tilde{F}_{2,0}-G_{2,0}\right) Q+\int_{\mathbb{R}} \tilde{F}_{2,0} A_{1,0}+\frac{1}{2} \int_{\mathbb{R}} f^{\prime \prime}(Q)\left(1+A_{1,0}\right)^{2}\right] .
$$

It is clear that

$$
\begin{aligned}
\tilde{F}_{2,0}-G_{2,0}= & \frac{3}{2} a_{1,0}^{2} Q^{\prime \prime}+a_{1,0}\left[\frac{3}{2} A_{1,0}^{\prime \prime}-f^{\prime}(Q)\left(1+A_{1,0}\right)+\frac{3}{2} B_{1,0}^{(3)}+\frac{1}{2}\left(f^{\prime}(Q) B_{1,0}\right)^{\prime}\right] \\
& -\frac{1}{2}\left(f^{\prime \prime}(Q)\left(1+A_{1,0}\right) B_{1,0}\right)^{\prime} .
\end{aligned}
$$

From here, after several integration by parts,

$$
\begin{aligned}
\int_{\mathbb{R}}\left(\tilde{F}_{2,0}-G_{2,0}\right) Q= & -\frac{3}{2} a_{1,0}^{2} \int_{\mathbb{R}} Q^{2}+a_{1,0} \int_{\mathbb{R}}\left[\frac{3}{2} A_{1,0}^{\prime \prime}-f^{\prime}(Q)\left(1+A_{1,0}\right)\right] Q \\
& -\frac{1}{2} a_{1,0} \int_{\mathbb{R}} B_{1,0}\left[3 Q^{\prime \prime}+f(Q)\right]^{\prime}+\frac{1}{2} \int_{\mathbb{R}} B_{1,0}\left(f^{\prime}(Q)\right)^{\prime}\left(1+A_{1,0}\right) \\
= & -\frac{3}{2} a_{1,0}^{2} \int_{\mathbb{R}} Q^{\prime 2}+a_{1,0} \int_{\mathbb{R}}\left[\frac{3}{2} A_{1,0}^{\prime \prime}-f^{\prime}(Q)\left(1+A_{1,0}\right)\right] Q \\
& +\frac{1}{2} \int_{\mathbb{R}} B_{1,0}\left[\left(f^{\prime}(Q)\right)^{\prime}\left(1+A_{1,0}\right)-a_{1,0}(3 Q-2 f(Q))^{\prime}\right] .
\end{aligned}
$$

But from (45), $\left(\mathcal{L}\left(1+A_{1,0}\right)\right)^{\prime}=\left(1-f^{\prime}(Q)+\mathcal{L} A_{1,0}\right)^{\prime}=-a_{1,0}(3 Q-2 f(Q))^{\prime}$. On the other hand, expanding $\left(\mathcal{L}\left(1+A_{1,0}\right)\right)^{\prime}$, we get

$$
\left(\mathcal{L}\left(1+A_{1,0}\right)\right)^{\prime}=-A_{1,0}^{(3)}+A_{1,0}^{\prime}-\left(f^{\prime}(Q)\right)^{\prime}\left(1+A_{1,0}\right)-f^{\prime}(Q) A_{1,0}^{\prime} .
$$


From here, the quantity in front of $B_{1,0}$ in (147) is nothing but $\mathcal{L} A_{1,0}^{\prime}$. Coming back to (147), and using the equation for $B_{1,0}$ (46), we obtain

$$
\begin{aligned}
\int_{\mathbb{R}}\left(\tilde{F}_{2,0}-G_{2,0}\right) Q= & -\frac{3}{2} a_{1,0}^{2} \int_{\mathbb{R}} Q^{\prime 2}+a_{1,0} \int_{\mathbb{R}}\left[\frac{3}{2} A_{1,0}^{\prime \prime}-f^{\prime}(Q)\left(1+A_{1,0}\right)\right] Q-\frac{1}{2} \int_{\mathbb{R}} A_{1,0}\left(\mathcal{L} B_{1,0}\right)^{\prime} \\
= & -\frac{3}{2} a_{1,0}^{2} \int_{\mathbb{R}} Q^{\prime 2}+\frac{3}{2} \int_{\mathbb{R}} A_{1,0}^{\prime 2}+3 a_{1,0} \int_{\mathbb{R}} Q^{\prime \prime} A_{1,0} \\
& -a_{1,0} \int_{\mathbb{R}} f^{\prime}(Q) Q\left(1+A_{1,0}\right)-\frac{1}{2} \int_{\mathbb{R}} f^{\prime}(Q)\left(1+A_{1,0}\right) A_{1,0} .
\end{aligned}
$$

Finally, an easy computation shows that

$$
\begin{aligned}
\int_{\mathbb{R}} \tilde{F}_{2,0} A_{1,0}= & \frac{1}{2} \int_{\mathbb{R}} f^{\prime \prime}(Q)\left(1+A_{1,0}\right)^{2} A_{1,0}+3 a_{1,0}^{2} \int_{\mathbb{R}} A_{1,0} Q^{\prime \prime} \\
& -a_{1,0} \int_{\mathbb{R}}\left(3 A_{1,0}^{\prime \prime}+f^{\prime}(Q)\left(1+A_{1,0}\right)\right) A_{1,0}
\end{aligned}
$$

Collecting (148) and (149), we get

$$
\begin{aligned}
b_{2,0}= & \frac{1}{4} \int_{\mathbb{R}} f^{\prime \prime}(Q)\left(1+A_{1,0}\right)^{3}-\frac{3}{4} a_{1,0} \int_{\mathbb{R}} f^{\prime}(Q)\left(1+A_{1,0}\right) A_{1,0}+\frac{9}{4} a_{1,0} \int_{\mathbb{R}} A_{1,0}^{\prime 2} \\
& -\frac{1}{2} a_{1,0}^{2} \int_{\mathbb{R}} f^{\prime}(Q) Q\left(1+A_{1,0}\right)+3 a_{1,0}^{2} \int_{\mathbb{R}} A_{1,0} Q^{\prime \prime}-\frac{3}{4} a_{1,0}^{3} \int_{\mathbb{R}} Q^{\prime 2} .
\end{aligned}
$$

as desired.

Let us treat now the quadratic case. The procedure is similar, but more involved. Now we assume that $\gamma_{2,0}=-\frac{1}{2} b_{1,0}^{2}$ as in Proposition 2.6 (i), and consider (58)-(59). We get

$$
b_{2,0}=\frac{1}{2}\left[-\frac{1}{2} b_{1,0}^{2} \int_{\mathbb{R}}\left(\bar{P}-a_{1,0} P\right)+\int_{\mathbb{R}} F_{2,0} \int_{0}^{x}\left(a_{1,0} P-\bar{P}\right)+\int_{\mathbb{R}} G_{2,0}-a_{1,0} \int_{\mathbb{R}} G_{2,0} Q\right] .
$$

Now several remarks. Note that from (48) and the definition of $P$ in (38) we have $\bar{P}-a_{1,0} P=$ $A_{1,0}+a_{1,0} Q$, and from (49),

$$
\int_{\mathbb{R}}\left(\bar{P}-a_{1,0} P\right)=2 b_{1,0} .
$$

Second, note that from $b_{1,0}= \pm \lim _{ \pm \infty} B_{1,0}$ and $\lim _{ \pm \infty} f^{\prime \prime}(Q)=2$,

$$
\int_{\mathbb{R}} G_{2,0}=\frac{1}{2} \int_{\mathbb{R}}\left(f^{\prime \prime}(Q)-2\right)-\int_{\mathbb{R}} A_{1,0}-4 b_{1,0}+\frac{1}{2} \int_{\mathbb{R}} f^{\prime \prime}(Q)\left(2 A_{1,0}+A_{1,0}^{2}\right) .
$$

On the other hand, from (52),$F_{2,0}=\tilde{F}_{2,0}^{\prime}-f^{\prime}(Q) B_{1,0}$, where $\tilde{F}_{2,0} \in \mathcal{Y}$ and is given by

$$
\begin{aligned}
\tilde{F}_{2,0}:=- & \left(3 A_{1,0}+3 B_{1,0}^{\prime}\right)+\frac{1}{2} f^{\prime \prime}(Q)\left(2 A_{1,0}+A_{1,0}^{2}\right) \\
& \quad-a_{1,0}\left(3 A_{1,0}^{\prime \prime}-Q+f^{\prime}(Q)\left(1+A_{1,0}\right)\right)+3 a_{1,0}^{2} Q^{\prime \prime}+\frac{1}{2}\left(f^{\prime \prime}(Q)-2\right) .
\end{aligned}
$$

Thus,

$$
\begin{aligned}
\int_{\mathbb{R}} F_{2,0} \int_{0}^{x}\left(a_{1,0} P-\bar{P}\right) & =\int_{\mathbb{R}} \tilde{F}_{2,0}\left(\bar{P}-a_{1,0} P\right)+\int_{\mathbb{R}} f^{\prime}(Q) B_{1,0} \int_{0}^{x}\left(\bar{P}-a_{1,0} P\right) \\
& =\int_{\mathbb{R}} \tilde{F}_{2,0}\left(A_{1,0}+a_{1,0} Q\right)+\int_{\mathbb{R}} f^{\prime}(Q) B_{1,0} \int_{0}^{x}\left(A_{1,0}+a_{1,0} Q\right) .
\end{aligned}
$$

Repeating the same computation for the cubic case, we obtain

$$
\begin{aligned}
\int_{\mathbb{R}} Q\left(\tilde{F}_{2,0}-G_{2,0}\right)= & -2 \int_{\mathbb{R}} Q A_{1,0}-\frac{3}{2} a_{1,0}^{2} \int_{\mathbb{R}} Q^{\prime 2}+a_{1,0} \int_{\mathbb{R}} Q\left(\frac{3}{2} A_{1,0}^{\prime \prime}+Q-f^{\prime}(Q)\left(1+A_{1,0}\right)\right) \\
& -\frac{1}{2} \int_{\mathbb{R}}\left[f^{\prime}(Q)\left(1+A_{1,0}\right)+3 A_{1,0}^{\prime \prime}-3 a_{1,0} Q^{\prime \prime}\right] A_{1,0},
\end{aligned}
$$


and

$$
\begin{aligned}
\int_{\mathbb{R}} \tilde{F}_{2,0} A_{1,0}=- & \int_{\mathbb{R}} A_{1,0}\left(3 A_{1,0}+3 B_{1,0}^{\prime}\right)+\frac{1}{2} \int_{\mathbb{R}} f^{\prime \prime}(Q)\left(2 A_{1,0}+A_{1,0}^{2}\right) A_{1,0}+3 a_{1,0}^{2} \int_{\mathbb{R}} Q^{\prime \prime} A_{1,0} \\
& -a_{1,0} \int_{\mathbb{R}}\left(3 A_{1,0}^{\prime \prime}-Q+f^{\prime}(Q)\left(1+A_{1,0}\right)\right) A_{1,0}+\frac{1}{2} \int_{\mathbb{R}}\left(f^{\prime \prime}(Q)-2\right) A_{1,0} .
\end{aligned}
$$

Collecting the above identities and after several simplifications we get

$$
\begin{aligned}
b_{2,0}= & -\frac{1}{2} b_{1,0}^{3}+\frac{1}{4} \int_{\mathbb{R}}\left(f^{\prime \prime}(Q)-2\right)\left(1+A_{1,0}\right)^{3}-2 b_{1,0}+\frac{1}{2} \int_{\mathbb{R}} A_{1,0}\left(1+A_{1,0}^{2}\right) \\
& -\frac{1}{2} a_{1,0} \int_{\mathbb{R}} Q A_{1,0}-\frac{3}{4} a_{1,0}^{3} \int_{\mathbb{R}} Q^{\prime 2}+\frac{1}{2} a_{1,0}^{2} \int_{\mathbb{R}} Q\left(Q-f^{\prime}(Q)\left(1+A_{1,0}\right)\right) \\
& -\frac{3}{4} a_{1,0} \int_{\mathbb{R}}\left[f^{\prime}(Q)\left(1+A_{1,0}\right)+3 A_{1,0}^{\prime \prime}\right] A_{1,0}+\frac{1}{2} \int_{\mathbb{R}} B_{1,0}\left[3 A_{1,0}^{\prime}+f^{\prime}(Q) \int_{0}^{x}\left(A_{1,0}+a_{1,0} Q\right)\right] \\
& +3 a_{1,0}^{2} \int_{\mathbb{R}} Q^{\prime \prime} A_{1,0} .
\end{aligned}
$$

The proof is now complete.

The objective is now to give the first order terms for the coefficient $b_{2,0}$. For this, we consider separate cases. It turns out that computations in the cubic case are easy to carry out. We first deal with this case.

B.2. Cubic case. The objective of this paragraph is to prove the following

Lemma B.1 (Asymptotic expansions, case $m=3$ ). We have

$$
b_{2,0}=b_{2,0}^{1} \varepsilon+o(\varepsilon) \text {. }
$$

where

$$
b_{2,0}^{1}=: c_{3, p}=-\left[\frac{(p-1)(p-3)\left(p^{2}-3 p+8\right)}{8(p-2)(p+1)}\right] \int_{\mathbb{R}}\left(Q^{0}\right)^{p} .
$$

In particular, for any $p \geq 4, b_{2,0}(\varepsilon) \neq 0$ provided $0<|\varepsilon| \leq \varepsilon_{0}$ for $\varepsilon_{0}$ small.

First of all we start with an auxiliary

Claim 12 (Asymptotic expansions, basic functions). Suppose $f$ as in (22), $p \geq 4$. The following asymptotic expansions hold.

(1) The soliton solution $Q$ can be expanded as

$$
Q=Q^{0}+\varepsilon Q^{1}+o(\varepsilon), \quad o(\varepsilon) \in \mathcal{Y},
$$

where $Q^{0}$ and $Q^{1}$ satisfy the equations

$$
-\left(Q^{0}\right)^{\prime \prime}+Q^{0}-\left(Q^{0}\right)^{3}=0, \quad \mathcal{L}^{0} Q^{1}:=-\left(Q^{1}\right)^{\prime \prime}+Q^{1}-3\left(Q^{0}\right)^{2} Q^{1}=\left(Q^{0}\right)^{p} .
$$

Finally,

$$
\left\{\begin{array}{l}
f(Q)=\left(Q^{0}\right)^{3}+\varepsilon\left(3\left(Q^{0}\right)^{2} Q^{1}+\left(Q^{0}\right)^{p}\right)+o(\varepsilon), \\
f^{\prime}(Q)=3\left(Q^{0}\right)^{2}+\varepsilon\left(6 Q^{0} Q^{1}+p\left(Q^{0}\right)^{p-1}\right)+o(\varepsilon), \\
f^{\prime \prime}(Q)=6 Q^{0}+\varepsilon\left(6 Q^{1}+p(p-1)\left(Q^{0}\right)^{p-2}\right)+o(\varepsilon),
\end{array}\right.
$$

where every term $o(\varepsilon) \in \mathcal{Y}$ uniformly in $\varepsilon<\varepsilon_{0}$.

(2) The operator $\mathcal{L}$ satisfies

$$
\mathcal{L}=\mathcal{L}^{0}-\varepsilon\left[6 Q^{0} Q^{1}+p\left(Q^{0}\right)^{p-1}\right]+o(\varepsilon), \quad \mathcal{L}^{0}=-\partial_{x}^{2}+1-3\left(Q^{0}\right)^{2} .
$$

(3) From (38), (39) and (42), the test functions $P, \bar{P}$ and $\hat{P}$ satisfy the following relations

$$
\left\{\begin{array}{l}
\Lambda Q=\Lambda Q^{0}+\varepsilon \Lambda Q^{1}+o(\varepsilon) \in \mathcal{Y} \text { where } \Lambda Q^{0}:=\frac{1}{2}\left(x\left(Q^{0}\right)^{\prime}+Q^{0}\right), \quad \mathcal{L}^{0} \Lambda Q^{0}=-Q^{0} \\
\text { and } \mathcal{L}^{0} \Lambda Q^{1}:=\left(6 Q^{0} \Lambda Q^{0}-1\right) Q^{1}+p\left(Q^{0}\right)^{p-1} \Lambda Q^{0} .
\end{array}\right.
$$


Moreover, the following identities hold

$$
\begin{aligned}
\int_{\mathbb{R}} \Lambda Q^{0}= & 0 \\
\int_{\mathbb{R}} \Lambda Q^{1}= & \int_{\mathbb{R}}\left[-1+\left(Q^{0}\right)^{2}+6 Q^{0} \Lambda Q^{0}-6\left(Q^{0}\right)^{3} \Lambda Q^{0}\right] Q^{1} \\
& +p \int_{\mathbb{R}}\left(Q^{0}\right)^{p-1} \Lambda Q^{0}\left(1-\left(Q^{0}\right)^{2}\right) .
\end{aligned}
$$

(4) Integrals. For any $p \geq 1$,

$$
\int_{\mathbb{R}}\left(Q^{0}\right)^{p+2}=\frac{2 p}{1+p} \int_{\mathbb{R}}\left(Q^{0}\right)^{p}, \quad \int_{\mathbb{R}}\left(Q^{0}\right)^{2}=4 .
$$

(5) Let $D(\varepsilon)=\int_{\mathbb{R}} \Lambda Q Q$. Then

$$
D(\varepsilon)=1+O(\varepsilon) .
$$

(6) Inverse functions. The following identities hold

$$
\begin{gathered}
\mathcal{L}^{0}\left(-\frac{9}{4} x\left(Q^{0}\right)^{\prime}-\frac{15}{4} Q^{0}+\frac{3}{2}\left(Q^{0}\right)^{3}\right)=\frac{9}{2} Q^{0}\left(1-\left(Q^{0}\right)^{2}\right)^{2}, \\
\mathcal{L}^{0}\left(x Q^{0}\left(Q^{0}\right)^{\prime}\right)=-4\left(Q^{0}\right)^{2}+3\left(Q^{0}\right)^{4}-3 x Q^{0}\left(Q^{0}\right)^{\prime}\left(1-\left(Q^{0}\right)^{2}\right), \\
\mathcal{L}^{0}\left(\left(Q^{0}\right)^{4}\right)=-15\left(Q^{0}\right)^{4}+7\left(Q^{0}\right)^{6} .
\end{gathered}
$$

Proof. First of all, (151)-(154) follow by Taylor expansion in $\varepsilon$. Concerning (155), it follows from (151)-(154). Let us see (156). From the definition of $\mathcal{L}^{0} \Lambda Q^{1}$ and the identity $\mathcal{L}^{0}\left(Q^{0}\right)^{2}=-3\left(Q^{0}\right)^{2}$, we have

thus

$$
\int_{\mathbb{R}} \mathcal{L}^{0} \Lambda Q^{1}=\int_{\mathbb{R}} \Lambda Q^{1}+\int_{\mathbb{R}}\left(Q^{0}\right)^{2} \mathcal{L}^{0} \Lambda Q^{1}
$$

$$
\int_{\mathbb{R}} \Lambda Q^{1}=\int_{\mathbb{R}}\left[1-\left(Q^{0}\right)^{2}\right] \mathcal{L}^{0} \Lambda Q^{1}=\int_{\mathbb{R}}\left[1-\left(Q^{0}\right)^{2}\right]\left[\left(6 Q^{0} \Lambda Q^{0}-1\right) Q^{1}+p\left(Q^{0}\right)^{p-1} \Lambda Q^{0}\right],
$$

where we obtain (156).

To obtain (157) we use integration by parts and the explicit function $Q^{0}(x):=\frac{\sqrt{2}}{\cosh x}$.

We prove (158). It follows from the fact that

$$
\int_{\mathbb{R}} Q^{0} \Lambda Q^{0}=\frac{1}{2} \int_{\mathbb{R}}\left(\frac{1}{2} x\left(\left(Q^{0}\right)^{2}\right)^{\prime}+\left(Q^{0}\right)^{2}\right)=\frac{1}{4} \int_{\mathbb{R}}\left(Q^{0}\right)^{2}=1 .
$$

Finally, (159)-(161) are obtained by simple differentiation. We left the proof to the reader.

Claim 13 (Asymptotic expansions, case $m=3$ ). The following expansion hold.

$$
\left\{\begin{array}{l}
a_{1,0}=a_{1,0}^{0}+\varepsilon a_{1,0}^{1}+o(\varepsilon), \quad a_{1,0}^{0}=0, \\
A_{1,0}=A_{1,0}^{0}+\varepsilon A_{1,0}^{1}+o(\varepsilon), \quad o(\varepsilon) \in \mathcal{Y}, \quad A_{1,0}^{0}=-\left(Q^{0}\right)^{2}, \\
B_{1,0}=B_{1,0}^{0}+\varepsilon B_{1,0}^{1}+o(\varepsilon), \quad B_{1,0}^{0}=-2 \varphi^{0}-\frac{3}{4} \sqrt{2} \pi\left(Q^{0}\right)^{\prime} .
\end{array}\right.
$$

Here $a_{1,0}^{1}:=\int_{\mathbb{R}} \Lambda Q^{1}$ and $A_{1,0}^{1}, B_{1,0}^{1}$ satisfy the following linear system

$$
\left\{\begin{array}{l}
\left(\mathcal{L}^{0} A_{1,0}^{1}\right)^{\prime}+a_{1,0}^{1}\left(3 Q^{0}-2\left(Q_{0}\right)^{3}\right)^{\prime}=\left(\left(6 Q^{0} Q^{1}+p\left(Q^{0}\right)^{p-1}\right)\left(1+A_{1,0}^{0}\right)\right)^{\prime}, \\
\left(\mathcal{L}^{0} B_{1,0}^{1}\right)^{\prime}+3 a_{1,0}^{1}\left(Q^{0}\right)^{\prime \prime}-3\left(A_{1,0}^{1}\right)^{\prime \prime}-3\left(Q^{0}\right)^{2} A_{1,0}^{1}=\left(6 Q^{0} Q^{1}+p\left(Q^{0}\right)^{p-1}\right)\left(1+A_{1,0}^{0}\right) .
\end{array}\right.
$$

Proof. We start with the zeroth order system. From (45)-(46) and using Claim 12 we get

$$
\left\{\begin{array}{l}
\left(\mathcal{L}^{0} A_{1,0}^{0}\right)^{\prime}+a_{1,0}^{0}\left(3 Q^{0}-2\left(Q^{0}\right)^{3}\right)^{\prime}=\left(3\left(Q^{0}\right)^{2}\right)^{\prime} . \\
\left(\mathcal{L}^{0} B_{1,0}^{0}\right)^{\prime}+3 a_{1,0}^{0}\left(Q^{0}\right)^{\prime \prime}-3\left(A_{1,0}^{0}\right)^{\prime \prime}-3\left(Q^{0}\right)^{2} A_{1,0}^{0}=3\left(Q^{0}\right)^{2} .
\end{array}\right.
$$

It is easy to verify that $a_{1,0}^{0}=0, A_{1,0}^{0}=-\left(Q^{0}\right)^{2} \in \mathcal{Y}$ and $B_{1,0}^{0}=-2 \varphi^{0}-\frac{3}{4} \sqrt{2} \pi\left(Q^{0}\right)^{\prime}$ satisfy this system with the required properties. In particular,

$$
\int_{\mathbb{R}} B_{1,0}^{0}\left(Q^{0}\right)^{\prime}=0 .
$$


Concerning the system (163), it follows directly from (50)-(51) and using Claim 12, We will not solve this system explicitly, but we only compute the constant $a_{1,0}^{1}$.

Indeed, from (47) and Claim 12, we have $a_{1,0}=a_{1,0}^{0}+a_{1,0}^{1} \varepsilon+o(\varepsilon)$, where

$$
a_{1,0}^{0}:=\frac{\int_{\mathbb{R}} \Lambda Q^{0}}{\int_{\mathbb{R}} \Lambda Q^{0} Q^{0}}=0, \quad \text { and } \quad a_{1,0}^{1}:=\frac{\int_{\mathbb{R}} \Lambda Q^{1}}{\int_{\mathbb{R}} \Lambda Q^{0} Q^{0}}=\int_{\mathbb{R}} \Lambda Q^{1} .
$$

This finishes the proof.

We finally prove Lemma B.1

Proof of Lemma B.1. From (144) and (162) we have $b_{2,0}=b_{2,0}^{0}+\varepsilon b_{2,0}^{1}+o(\varepsilon)$, where

$$
b_{2,0}^{0}=\frac{1}{4} \int_{\mathbb{R}} 6 Q^{0}\left(1+A_{1,0}^{0}\right)^{3}=\frac{1}{4} \int_{\mathbb{R}} 6 Q^{0}\left(1-\left(Q^{0}\right)^{2}\right)^{3}=0,
$$

and

$$
\begin{aligned}
b_{2,0}^{1}= & \frac{1}{4} \int_{\mathbb{R}}\left(6 Q^{1}+p(p-1)\left(Q^{0}\right)^{p-2}\right)\left(1-\left(Q^{0}\right)^{2}\right)^{3}+\frac{9}{2} \int_{\mathbb{R}} Q^{0}\left(1-\left(Q^{0}\right)^{2}\right)^{2} A_{1,0}^{1} \\
& +\frac{9}{4} a_{1,0}^{1}\left[\int_{\mathbb{R}}\left(Q^{0}\right)^{2}\left(1-\left(Q^{0}\right)^{2}\right)\left(Q^{0}\right)^{2}+\int_{\mathbb{R}} 4\left(Q^{0}\right)^{2}\left(\left(Q^{0}\right)^{2}-\frac{1}{2}\left(Q^{0}\right)^{4}\right)\right] .
\end{aligned}
$$

From (159), the selfadjointness of the operator $\mathcal{L}^{0}$ and by using (163), we get

$$
\begin{aligned}
\left.\frac{9}{2} \int_{\mathbb{R}} Q^{0}\left(1-\left(Q^{0}\right)^{2}\right)\right)^{2} A_{1,0}^{1}= & \frac{3}{4} \int_{\mathbb{R}}\left(-3 x\left(Q^{0}\right)^{\prime}-5 Q^{0}+2\left(Q^{0}\right)^{3}\right) \mathcal{L}^{0} A_{1,0}^{1} \\
= & \frac{3}{4} a_{1,0}^{1} \int_{\mathbb{R}}\left(3 x\left(Q^{0}\right)^{\prime}+5 Q^{0}-2\left(Q^{0}\right)^{3}\right)\left(3 Q^{0}-2\left(Q^{0}\right)^{3}\right) \\
& -\frac{3}{4} \int_{\mathbb{R}}\left(3 x\left(Q^{0}\right)^{\prime}+5 Q^{0}-2\left(Q^{0}\right)^{3}\right)\left(6 Q^{0} Q^{1}+p\left(Q^{0}\right)^{p-1}\right)\left(1-\left(Q^{0}\right)^{2}\right) .
\end{aligned}
$$

Therefore,

$$
\begin{aligned}
b_{2,0}^{1}= & \frac{3}{2} \int_{\mathbb{R}} Q^{1}\left(1-\left(Q^{0}\right)^{2}\right)\left[1-2\left(Q^{0}\right)^{2}+\left(Q^{0}\right)^{4}-3 Q^{0}\left(3 x\left(Q^{0}\right)^{\prime}+5 Q^{0}-2\left(Q^{0}\right)^{3}\right)\right] \\
& +\frac{p}{4} \int_{\mathbb{R}}\left(Q^{0}\right)^{p-2}\left(1-\left(Q^{0}\right)^{2}\right)\left[(p-1)\left(1-2\left(Q^{0}\right)^{2}+\left(Q^{0}\right)^{4}\right)-3 Q^{0}\left(3 x\left(Q^{0}\right)^{\prime}+5 Q^{0}-2\left(Q^{0}\right)^{3}\right)\right] \\
& +\frac{3}{4} \int_{\mathbb{R}} \Lambda Q^{1}\left[\int_{\mathbb{R}}\left(3 x\left(Q^{0}\right)^{\prime}+5 Q^{0}-2\left(Q^{0}\right)^{3}\right)\left(3 Q^{0}-2\left(Q^{0}\right)^{3}\right)+3 \int_{\mathbb{R}}\left(Q^{0}\right)^{4}\left(5-3\left(Q^{0}\right)^{2}\right)\right] .
\end{aligned}
$$

Note that, from (157)

$$
\int_{\mathbb{R}}\left(3 x\left(Q^{0}\right)^{\prime}+5 Q^{0}-2\left(Q^{0}\right)^{3}\right)\left(3 Q^{0}-2\left(Q^{0}\right)^{3}\right)+3 \int_{\mathbb{R}}\left(Q^{0}\right)^{4}\left(5-3\left(Q^{0}\right)^{2}\right)=2,
$$

thus from (156) we get

$$
\begin{aligned}
b_{2,0}^{1}= & \frac{3}{2} \int_{\mathbb{R}} Q^{1}\left(1-\left(Q^{0}\right)^{2}\right)\left[-14\left(Q^{0}\right)^{2}+7\left(Q^{0}\right)^{4}-6 x Q^{0}\left(Q^{0}\right)^{\prime}\right] \\
& +\frac{p}{4} \int_{\mathbb{R}}\left(Q^{0}\right)^{p-2}\left(1-\left(Q^{0}\right)^{2}\right)\left[(p-1)\left(1-2\left(Q^{0}\right)^{2}+\left(Q^{0}\right)^{4}\right)-3 Q^{0}\left(3 x\left(Q^{0}\right)^{\prime}+5 Q^{0}-2\left(Q^{0}\right)^{3}\right)\right] \\
& +\frac{3}{2} p \int_{\mathbb{R}}\left(Q^{0}\right)^{p-1} \Lambda Q^{0}\left(1-\left(Q^{0}\right)^{2}\right)
\end{aligned}
$$

Finally, from (160), (161) and the identity $\mathcal{L}^{0}\left(Q^{0}\right)^{2}=-3\left(Q^{0}\right)^{2}$, we have

$$
\mathcal{L}^{0}\left[2\left(Q^{0}\right)^{2}-\left(Q^{0}\right)^{4}+2 x Q^{0}\left(Q^{0}\right)^{\prime}\right]=\left(1-\left(Q^{0}\right)^{2}\right)\left[-14\left(Q^{0}\right)^{2}+7\left(Q^{0}\right)^{4}-6 x Q^{0}\left(Q^{0}\right)^{\prime}\right] .
$$


Using the selfadjointness of $\mathcal{L}^{0}$ and the equation for $Q^{1}$ in (152), and after integrating by parts, we conclude that

$$
\begin{aligned}
b_{2,0}^{1}= & \frac{3}{2} \int_{\mathbb{R}}\left(Q^{0}\right)^{p}\left(2\left(Q^{0}\right)^{2}-\left(Q^{0}\right)^{4}+2 x Q^{0}\left(Q^{0}\right)^{\prime}\right)+\frac{3}{2} p \int_{\mathbb{R}}\left(Q^{0}\right)^{p-1} \Lambda Q^{0}\left(1-\left(Q^{0}\right)^{2}\right) \\
& +\frac{p}{4} \int_{\mathbb{R}}\left(Q^{0}\right)^{p-2}\left(1-\left(Q^{0}\right)^{2}\right)\left[(p-1)\left(1-2\left(Q^{0}\right)^{2}+\left(Q^{0}\right)^{4}\right)-3 Q^{0}\left(3 x\left(Q^{0}\right)^{\prime}+5 Q^{0}-2\left(Q^{0}\right)^{3}\right)\right] \\
= & \frac{p}{4}(p-1) \int_{\mathbb{R}}\left(Q^{0}\right)^{p-2}-\frac{3}{4}\left(p^{2}+3 p-2\right) \int_{\mathbb{R}}\left(Q^{0}\right)^{p}+\frac{3}{4(p+2)}\left(p^{3}+7 p^{2}+12 p+4\right) \int_{\mathbb{R}}\left(Q^{0}\right)^{p+2} \\
& -\frac{1}{4}\left(p^{2}+5 p+6\right) \int_{\mathbb{R}}\left(Q^{0}\right)^{p+4} .
\end{aligned}
$$

Finally, from (157), and after some simplifications,

$$
b_{2,0}^{1}=-\left[\frac{(p-1)(p-3)\left(p^{2}-3 p+8\right)}{8(p-2)(p+1)}\right] \int_{\mathbb{R}}\left(Q^{0}\right)^{p} .
$$

The proof is now complete.

Remark B.1. Note that even though the higher regularity needed in our results $\left(f \in C^{5}\right.$ for $m=3$ ), we are able to take, at least formally, the limit $p \downarrow 3$ in (164), recovering the results from the integrable case (that is, $b_{2,0}^{1}=0$ ). This gain of regularity comes from (54) and (144): for these identities, we only need $f \in C^{3}(\mathbb{R})$.

B.3. Gardner and quadratic nonlinearities. These two nonlinearities are very similar to handle. Although computations are harder for the Gardner nonlinearity, a simple trick will allow to link both results. As a consequence, we are reduced to consider only the quadratic case.

Finally, recall the soliton $Q_{\tilde{\mu}, 1}$ introduced in (6), well defined for $\tilde{\mu}<\frac{2}{9}$. Given $\tilde{\mu}, \nu \in \mathbb{R}$, $\tilde{\mu}<\frac{2}{9}$ and $\nu$ small enough, let $d_{\tilde{\mu}, \nu}$ be the defect (possibly zero) associated the the nonlinearity $f_{\tilde{\mu}, \nu}(s):=s^{2}-\tilde{\mu} s^{3}+\nu s^{p}$, namely

$$
d_{\tilde{\mu}, \nu}:=b_{2,0}\left(f_{\tilde{\mu}, \nu}\right)+\frac{1}{6} b_{1,0}^{3}\left(f_{\tilde{\mu}, \nu}\right) .
$$

We following reduction Lemma is the key ingredient of the proof.

\section{Lemma B.2.}

Let $d(\varepsilon)$ be the defect parameter introduced in (56) for the nonlinearity $f(s)$ described in (20), $m=2$, and let $d_{\tilde{\mu}, \nu}$ be the defect introduced in (165), for $\tilde{\mu}, \nu$ small. Then the following properties are satisfied:

(1) For all $\tilde{\mu}<\frac{2}{9}, \nu \in \mathbb{R}$ small, $d_{\tilde{\mu}, \nu}$ is a smooth function of $\tilde{\mu}, \nu$ and for all $\tilde{\mu}<\frac{2}{9}$,

$$
d_{\tilde{\mu}, 0}=0 .
$$

(2) Given $\varepsilon$ small, let $\tilde{\mu}=\mu(\varepsilon)$ and $\nu=\varepsilon$. Then the following expansion holds

$$
d_{\mu(\varepsilon), \varepsilon}=-\varepsilon\left[\frac{(p-3)(2 p-1)\left(48-46 p+6 p^{2}+4 p^{3}\right)}{72\left(p^{2}-1\right)(p-2)}\right] \int_{\mathbb{R}}\left[\frac{3}{2 \cosh ^{2}(x / 2)}\right]^{p}+o(\varepsilon),
$$

for all $|\varepsilon|<\varepsilon_{0}$ and $p \geq 3$.

(3) The following expansion holds

$$
d(\varepsilon)=d_{\mu(\varepsilon), \varepsilon}+o(\varepsilon), \quad \text { as } \varepsilon \rightarrow 0 .
$$

Proof of (168). This is an easy consequence of the definition of $f$ in (21), and the fact that $f(Q)=$ $f_{\mu(\varepsilon), \varepsilon}(Q)+o(\varepsilon)$, with $o(\varepsilon) \in \mathcal{Y}$. In particular, the soliton $Q$ and each term $a_{1,0}, b_{1,0}, A_{1,0}, B_{1,0}$ and $b_{2,0}$ depends smoothly in $\varepsilon$ and can be expanded in a similar way. 
Proof of (166). The smoothness is a direct consequence of the formula for $b_{2,0}$ in Claim [11 and $b_{1,0}$ in (49). We have to prove that for all $\tilde{\mu}<\frac{2}{9}$,

$$
b_{2,0}\left(f_{\tilde{\mu}, 0}\right)+\frac{1}{6} b_{1,0}^{3}\left(f_{\tilde{\mu}, 0}\right)=0,
$$

In order to prove this identity, we claim the following

Claim 14 (Basic functions). Let $Q^{0}:=Q_{\tilde{\mu}, 1}$ be the soliton for the Gardner equation. Then we have

(1) The soliton solution $Q^{0}$ satisfies

$$
-\left(Q^{0}\right)^{\prime \prime}+Q^{0}-\left(Q^{0}\right)^{2}+\tilde{\mu}\left(Q^{0}\right)^{3}=0, \quad \mathcal{L}^{0}\left(Q^{0}\right)^{\prime}=0,
$$

where $\mathcal{L}^{0}:=-\partial_{x x}+1-\left(2 Q^{0}-3 \tilde{\mu}\left(Q^{0}\right)^{2}\right)$.

(2) From the definition of $Q^{0}$, we have

$$
\Lambda Q^{0}:=\frac{1}{2}\left(x\left(Q^{0}\right)^{\prime}+2 Q^{0}\right)+\frac{3 \tilde{\mu}}{4 \rho^{2}}\left(3 Q^{0}-\left(Q^{0}\right)^{2}\right), \quad \mathcal{L}^{0} \Lambda Q^{0}=-Q^{0} .
$$

Moreover,

$$
\int_{\mathbb{R}} \Lambda Q^{0}=\frac{3}{\rho^{2}}, \quad \int_{\mathbb{R}} \Lambda Q^{0} Q^{0}=\frac{9}{2 \rho^{2}} .
$$

(3) Resonance functions. Define $\varphi^{0}:=-\frac{\left(Q^{0}\right)^{\prime}}{Q^{0}}$. Then

$$
\left(\varphi^{0}\right)^{\prime}=\frac{1}{3} Q^{0}-\frac{\tilde{\mu}}{2}\left(Q^{0}\right)^{2}, \quad\left(\varphi^{0}\right)^{2}=1-\frac{2}{3} Q^{0}+\frac{\tilde{\mu}}{2}\left(Q^{0}\right)^{2} .
$$

(4) Integrals. For any $p \geq 1$,

$$
\tilde{\mu} \int_{\mathbb{R}}\left(Q^{0}\right)^{p+2}=\frac{2(2 p+1)}{3(1+p)} \int_{\mathbb{R}}\left(Q^{0}\right)^{p+1}-\frac{2 p}{p+1} \int_{\mathbb{R}}\left(Q^{0}\right)^{p} .
$$

Proof. A direct computation, see e.g. Claim 12 for a similar proof.

Now we proceed to give the explicit value the constants and functions related to system $\left(\Omega_{1,0}\right)$, see (45)-(46).

Claim 15 (Resolution of $\left(\Omega_{1,0}\right)$ for the Gardner equation). Denote by $\left(a_{1,0}^{0}, A_{1,0}^{0}, B_{1,0}^{0}\right)$ the solution of the linear system $\left(\Omega_{1,0}\right)$, for the Gardner nonlinearity. Then we have

$$
\left\{\begin{array}{l}
a_{1,0}^{0}=\frac{2}{3}, \\
A_{1,0}^{0}=-\frac{4}{3} Q^{0}+\tilde{\mu}\left(Q^{0}\right)^{2}, \quad B_{1,0}^{0}=-2 \varphi^{0}+\kappa_{1,0}^{0}\left(Q^{0}\right)^{\prime}, \\
b_{1,0}^{0}=\lim _{+\infty} B_{1,0}^{0}=-2,
\end{array}\right.
$$

with

$$
\kappa_{1,0}^{0}=\frac{3 \tilde{\mu}\left(\int_{\mathbb{R}}\left(Q^{0}\right)^{2}-3 \int_{\mathbb{R}} Q^{0}\right)}{(3 \tilde{\mu}-1) \int_{\mathbb{R}}\left(Q^{0}\right)^{2}+\int_{\mathbb{R}} Q^{0}}=-\frac{10}{3}+o_{\tilde{\mu}}(1) .
$$

Remark B.2. It is remarkable the similarity among the functions solution of the Gardner system $\left(\Omega_{1,0}\right)$ and the corresponding ones for the quadratic nonlinearity (let $\tilde{\mu} \rightarrow 0$ ).

Proof. First of all, the explicit value of $\left(a_{1,0}^{0}, A_{1,0}^{0}, B_{1,0}^{0}\right)$ comes from a straightforward verification. More precisely, this triplet is a solution of the zeroth order system

$$
\left\{\begin{array}{l}
\left(\mathcal{L}^{0} A_{1,0}^{0}\right)^{\prime}+a_{1,0}^{0}\left(3 Q^{0}-2\left(Q^{0}\right)^{2}+2 \tilde{\mu}\left(Q^{0}\right)^{3}\right)^{\prime}=\left(2 Q^{0}-3 \tilde{\mu}\left(Q^{0}\right)^{2}\right)^{\prime} \\
\left(\mathcal{L}^{0} B_{1,0}^{0}\right)^{\prime}+3 a_{1,0}^{0}\left(Q^{0}\right)^{\prime \prime}-3\left(A_{1,0}^{0}\right)^{\prime \prime}-\left(2 Q^{0}-3 \tilde{\mu}\left(Q^{0}\right)^{2}\right) A_{1,0}^{0}=2 Q^{0}-3 \tilde{\mu}\left(Q^{0}\right)^{2}
\end{array}\right.
$$

which comes from (45)-(46). In particular, we choose $\kappa_{1,0}$ such that $\int_{\mathbb{R}} B_{1,0}^{0}\left(Q^{0}\right)^{\prime}=0$. The value of $b_{1,0}^{0}$ comes from the fact that $b_{1,0}^{0}=-2 \lim _{+\infty} \varphi^{0}=-2$. On the other hand, from (49), one has

$$
b_{1,0}^{0}=-\frac{1}{3} \int_{\mathbb{R}} Q^{0}+\frac{1}{2} \tilde{\mu} \int_{\mathbb{R}}\left(Q^{0}\right)^{2}=-2 .
$$


Now we are able to prove (166). (Note that this is also a consequence of the integrability of the Gardner equation.) First, we claim that

$$
3\left(A_{1,0}^{0}\right)^{\prime}+\left(2 Q^{0}-3 \tilde{\mu}\left(Q^{0}\right)^{2}\right) \int_{0}^{x}\left(A_{1,0}^{0}+a_{1,0}^{0} Q^{0}\right)=3\left(A_{1,0}^{0}\right)^{\prime}-2\left(2 Q^{0}-3 \tilde{\mu}\left(Q^{0}\right)^{2}\right) \varphi^{0}=0 .
$$

This is an easy consequence of (170) and the values of of $A_{1,0}^{0}$ and $a_{1,0}^{0}$. Consider now the expression for $b_{2,0}, m=2$ in Claim 11, Note that the term containing $B_{1,0}^{0}$ disappears. Replacing the values of $a_{1,0}^{0}$ and $A_{1,0}^{0}$, and using the recursive formula (171), we have

$$
\begin{aligned}
d_{\tilde{\mu}, 0}=- & \frac{1}{3}\left(b_{1,0}^{0}\right)^{3}-\frac{3}{2} \tilde{\mu} \int_{\mathbb{R}} Q^{0}\left(1+A_{1,0}^{0}\right)^{3}-2 b_{1,0}^{0}+\frac{1}{2} \int_{\mathbb{R}} A_{1,0}^{0}\left(1+\left(A_{1,0}^{0}\right)^{2}\right)-\frac{1}{3} \int_{\mathbb{R}} Q^{0} A_{1,0}^{0} \\
& -\frac{2}{9} \int_{\mathbb{R}}\left(Q^{0}\right)^{\prime 2}+\frac{2}{9} \int_{\mathbb{R}}\left(Q^{0}\right)^{2}\left[1-\left(2-3 \tilde{\mu} Q^{0}\right)\left(1+A_{1,0}^{0}\right)\right]+\frac{4}{3} \int_{\mathbb{R}}\left(Q^{0}\right)^{\prime \prime} A_{1,0}^{0} \\
& \quad-\frac{1}{2} \int_{\mathbb{R}}\left[\left(2 Q^{0}-3 \tilde{\mu}\left(Q^{0}\right)^{2}\right)\left(1+A_{1,0}^{0}\right)+3\left(A_{1,0}^{0}\right)^{\prime \prime}\right] A_{1,0}^{0} \\
= & -\frac{1}{3}\left(b_{1,0}^{0}\right)^{3}-\frac{3}{2} \tilde{\mu} \int_{\mathbb{R}} Q^{0}+\left(\frac{11}{2} \tilde{\mu}-\frac{28}{9}\right) \int_{\mathbb{R}}\left(Q^{0}\right)^{2}+\left(-\frac{9}{2} \tilde{\mu}^{2}+\frac{2}{3} \tilde{\mu}+\frac{20}{9}\right) \int_{\mathbb{R}}\left(Q^{0}\right)^{3} \\
& +\tilde{\mu}\left(\frac{15}{2} \tilde{\mu}-\frac{13}{3}\right) \int_{\mathbb{R}}\left(Q^{0}\right)^{4}-\frac{9}{2} \tilde{\mu}^{3} \int_{\mathbb{R}}\left(Q^{0}\right)^{5}+\frac{7}{2} \tilde{\mu}^{3} \int_{\mathbb{R}}\left(Q^{0}\right)^{6}-\frac{3}{2} \tilde{\mu}^{4} \int_{\mathbb{R}}\left(Q^{0}\right)^{7} \\
= & -\frac{1}{3}\left(b_{1,0}^{0}\right)^{3}-\frac{4}{9} \int_{\mathbb{R}} Q^{0}+\frac{2}{3} \tilde{\mu} \int_{\mathbb{R}}\left(Q^{0}\right)^{2} \\
= & \frac{1}{3} b_{1,0}^{0}\left(4-\left(b_{1,0}^{0}\right)^{2}\right)=0 .
\end{aligned}
$$

This proves (166).

Proof of (167). The proof of (167) is a consequence of (166) and the simple relationship

$$
d_{\tilde{\mu}, \nu}=d_{\tilde{\mu}, 0}+\nu \partial_{\nu} d_{\tilde{\mu}, \nu}+o_{\nu}(\nu)=\nu\left(\partial_{\nu} d_{0, \nu}+o_{\tilde{\mu}}(1)\right)+o_{\nu}(\nu),
$$

valid for any $\tilde{\mu}, \nu \in \mathbb{R}$ small enough. This result says that, in order to prove (167), we only need to compute the defect for first order expansion in $\nu$ of the quadratic nonlinearity $f(s)=s^{2}+\nu s^{p}$, $p \geq 3$. Then we use the fact that $\tilde{\mu}=\mu(\varepsilon) \sim \varepsilon^{1 /(p-2)}$ and $\nu=\varepsilon$ to conclude. Consequently, in what follows we are reduced to prove that $\partial_{\nu} d_{0, \nu} \neq 0$ for all $\nu$ small enough.

Claim 16 (Asymptotic expansions, case $m=2$, basic functions). Suppose now $f(s)=f_{0, \nu}(s)=$ $s^{2}+\nu s^{p}$. Let $Q^{0}(x)=\frac{3}{2 \cosh ^{2}(x / 2)}$ be the soliton solution for the quadratic case. Then the following asymptotic expansions hold.

(1) The soliton solution $Q$ for the nonlinearity $f$ can be expanded as

$$
Q=Q^{0}+\nu Q^{1}+o(\nu), \quad o(\nu) \in \mathcal{Y},
$$

where $Q^{1}$ satisfies the equation $\mathcal{L}^{0} Q^{1}:=-\left(Q^{1}\right)^{\prime \prime}+Q^{1}-2 Q^{0} Q^{1}=\left(Q^{0}\right)^{p}$. We also have

$$
\left\{\begin{array}{l}
f(Q)=\left(Q^{0}\right)^{2}+\nu\left(\left(Q^{0}\right)^{p}+2 Q^{0} Q^{1}\right)+o(\nu), \\
f^{\prime}(Q)=2 Q^{0}+\nu\left(2 Q^{1}+p\left(Q^{0}\right)^{p-1}\right)+o(\nu), \\
f^{\prime \prime}(Q)=2+\nu p(p-1)\left(Q^{0}\right)^{p-2}+o(\nu),
\end{array}\right.
$$

where every term $o(\nu) \in \mathcal{Y}$ uniformly in $\nu<\nu_{0}$ small.

(2) The operator $\mathcal{L}$ satisfies

$$
\mathcal{L}=\mathcal{L}^{0}-\nu\left[2 Q^{1}+p\left(Q^{0}\right)^{p-1}\right]+o(\nu) .
$$

(3) From the definition of $Q$, we have

$$
\Lambda Q=\Lambda Q^{0}+\nu \Lambda Q^{1}+o(\nu), \quad \mathcal{L}^{0} \Lambda Q^{1}=-Q^{1}+\left(2 Q^{1}+p\left(Q^{0}\right)^{p-1}\right) \Lambda Q^{0} .
$$

(4) Let $D(\nu):=\int_{\mathbb{R}} \Lambda Q Q$. Then

$$
D(\nu)=\frac{9}{2}+\nu\left(\int_{\mathbb{R}} \Lambda Q^{1} Q^{0}+\int_{\mathbb{R}} \Lambda Q^{0} Q^{1}\right)+o(\nu) .
$$


(5) Inverse functions. We have

$$
\begin{gathered}
\mathcal{L}^{0}\left[1-\frac{4}{3} \Lambda Q^{0}\right]=1-\frac{2}{3} Q^{0} . \\
\mathcal{L}^{0}\left[\left(1-Q^{0}\right)\left(1+\frac{1}{3} x^{2} Q^{0}\right)-Q^{0}\right]=1-\frac{8}{3} \Lambda Q^{0}+\frac{8}{3}\left(\Lambda Q^{0}\right)^{2} . \\
\mathcal{L}^{0}\left[-5+\frac{68}{9} Q^{0}-6 \Lambda Q^{0}\right]=-5+16 Q^{0}-\frac{68}{9}\left(Q^{0}\right)^{2} . \\
\mathcal{L}^{0}\left[2+\frac{20}{3} \Lambda Q^{0}-\frac{170}{27} Q^{0}\right]=2-\frac{32}{3} Q^{0}+\frac{170}{27}\left(Q^{0}\right)^{2} .
\end{gathered}
$$

Proof. First of all, (173)-(175) are a direct consequence of a Taylor expansion of the considered functions. The expression for $\left(\Lambda Q^{0}\right)^{2}$ comes from a simple computation.

The expansion of $D(\nu)$ in (176) follows from a Taylor expansion and the fact that

$$
\int_{\mathbb{R}} \Lambda Q^{0} Q^{0}=\frac{3}{4} \int_{\mathbb{R}}\left(Q^{0}\right)^{2}=\frac{9}{2}
$$

Finally we prove (178), (179) and (180). These follow from the identities $\mathcal{L}^{0} 1=1-2 Q^{0}, \quad \mathcal{L}^{0} Q^{0}=$ $-\left(Q^{0}\right)^{2}, \mathcal{L}^{0} \Lambda Q^{0}=-Q^{0}, \mathcal{L}^{0}\left(x^{2} Q^{0}\right)=-2 Q^{0}-4 x\left(Q^{0}\right)^{\prime}-x^{2}\left(Q^{0}\right)^{2}$, and

$$
\mathcal{L}^{0}\left(x^{2}\left(Q^{0}\right)^{2}\right)=-2\left(Q^{0}\right)^{2}-8 x Q^{0}\left(Q^{0}\right)^{\prime}-3 x^{2}\left(Q^{0}\right)^{2}+\frac{4}{3} x^{2}\left(Q^{0}\right)^{3} .
$$

This finishes the proof.

Now we proceed to give an asymptotic expansion of the constants and functions related to system $\left(\Omega_{1,0}\right)$, see (45)-(46).

Claim 17 (Asymptotic expansions II, case $m=2$ ). There exists $\nu_{0}$ small enough such that for all $|\nu| \leq \nu_{0}$, the following holds. Let $f(s)=s^{2}+\nu s^{p}$, then the corresponding solution to the system $\left(\Omega_{1,0}\right)$ for this case can be expanded as follows:

$$
a_{1,0}=\frac{2}{3}+\nu a_{1,0}^{1}+o(\nu), \quad A_{1,0}=-\frac{4}{3} Q^{0}+\nu A_{1,0}^{1}+o(\nu) \in \mathcal{Y}, \quad b_{1,0}=-2+b_{1,0}^{1} \nu+o(\nu),
$$

where $\nu^{-1} o(\nu) \rightarrow 0$ as $\nu \rightarrow 0$ and $A_{1,0}^{1} \in \mathcal{Y}$ is a solution of the following linear equation

$$
\left(\mathcal{L}^{0} A_{1,0}^{1}\right)^{\prime}+a_{1,0}^{1}\left(3 Q^{0}-2\left(Q^{0}\right)^{2}\right)^{\prime}=\left[p\left(Q^{0}\right)^{p-1}-\frac{4}{3}(p-1)\left(Q^{0}\right)^{p}\right]^{\prime},
$$

Finally, the following two expressions are satisfied

$$
a_{1,0}^{1}=-\frac{1}{9}\left[\frac{(p-3)(2 p-1)}{p+1}\right] \int_{\mathbb{R}}\left(Q^{0}\right)^{p},
$$

and

$$
b_{1,0}^{1}=\frac{1}{2} \int_{\mathbb{R}} A_{1,0}^{1}+\frac{1}{2} a_{1,0}^{1} \int_{\mathbb{R}} Q^{0}+\frac{1}{3} \int_{\mathbb{R}} Q^{1} .
$$

Proof. The proof of (181) and (182) is direct from Claim 16 and (45)-(46). To prove (183), first note that from Claim 16

$$
\begin{aligned}
a_{1,0}^{1} & =\frac{2}{9}\left[\int_{\mathbb{R}}\left(1-\frac{2}{3} Q^{0}\right) \Lambda Q^{1}-\frac{2}{3} \int_{\mathbb{R}} Q^{1} \Lambda Q^{0}\right] \\
& =\frac{2}{9} \int_{\mathbb{R}}\left[1-\frac{4}{3} \Lambda Q^{0}\right]\left[-Q^{1}+\left(2 Q^{1}+p\left(Q^{0}\right)^{p-1}\right) \Lambda Q^{0}\right]-\frac{4}{27} \int_{\mathbb{R}} Q^{1} \Lambda Q^{0} \\
& =-\frac{2}{9} \int_{\mathbb{R}} Q^{1}\left[1-\frac{8}{3} \Lambda Q^{0}+\frac{8}{3}\left(\Lambda Q^{0}\right)^{2}\right]+\frac{2 p}{9} \int_{\mathbb{R}}\left(Q^{0}\right)^{p-1} \Lambda Q^{0}\left[1-\frac{4}{3} \Lambda Q^{0}\right] .
\end{aligned}
$$


Thus from (178) and Claim [16 (i), we get after integration by parts

$$
\begin{aligned}
a_{1,0}^{1}= & \frac{2}{9} \int_{\mathbb{R}}\left(Q^{0}\right)^{p-1}\left[p \Lambda Q^{0}\left(1-\frac{4}{3} \Lambda Q^{0}\right)-Q^{0}\left(1-Q^{0}\right)\left(1+\frac{1}{3} x^{2} Q^{0}\right)+\left(Q^{0}\right)^{2}\right] \\
= & \frac{2}{9}\left[\int_{\mathbb{R}}\left(Q^{0}\right)^{p-1}\left[(p-1) Q^{0}+\left(2-\frac{4}{3} p\right)\left(Q^{0}\right)^{2}+\frac{p}{2} x\left(Q^{0}\right)^{\prime}-\frac{4}{3} p x Q^{0}\left(Q^{0}\right)^{\prime}\right]\right. \\
& \left.\quad-\frac{1}{3} \int_{\mathbb{R}} x^{2}\left(Q^{0}\right)^{p+1}\left[(p+1)-\left(1+\frac{2}{3} p\right) Q^{0}\right]\right] \\
= & \frac{2}{9}\left[\left(p-\frac{3}{2}\right) \int_{\mathbb{R}}\left(Q^{0}\right)^{p}+\left(\frac{10}{3}-\frac{4}{3} p-\frac{4}{3} \frac{1}{p+1}\right) \int_{\mathbb{R}}\left(Q^{0}\right)^{p+1}\right. \\
& \left.\quad-\frac{1}{3} \int_{\mathbb{R}} x^{2}\left(Q^{0}\right)^{p+1}\left[(p+1)-\left(1+\frac{2}{3} p\right) Q^{0}\right]\right] .
\end{aligned}
$$

Now, recall that from the equation satisfied by $Q^{0},\left[\left(Q^{0}\right)^{p+1}\right]^{\prime \prime}=(p+1)\left(Q^{0}\right)^{p+1}\left[(p+1)-\left(1+\frac{2}{3} p\right) Q^{0}\right]$, so that

$$
\int_{\mathbb{R}} x^{2}\left(Q^{0}\right)^{p+1}\left[(p+1)-\left(1+\frac{2}{3} p\right) Q^{0}\right]=\frac{1}{p+1} \int_{\mathbb{R}} x^{2}\left[\left(Q^{0}\right)^{p+1}\right]^{\prime \prime}=\frac{2}{p+1} \int_{\mathbb{R}}\left(Q^{0}\right)^{p+1} .
$$

In conclusion, from (171),

$$
a_{1,0}^{1}=\frac{2}{9}\left[\left(p-\frac{3}{2}\right) \int_{\mathbb{R}}\left(Q^{0}\right)^{p}+\left(\frac{10}{3}-\frac{4}{3} p-\frac{2}{p+1}\right) \int_{\mathbb{R}}\left(Q^{0}\right)^{p+1}\right]=-\frac{1}{9}\left[\frac{(p-3)(2 p-1)}{p+1}\right] \int_{\mathbb{R}}\left(Q^{0}\right)^{p},
$$

as desired. Finally, from (49) we obtain (184).

Now we deal with the second order system $\left(\Omega_{2,0}\right)$ written in (50), (51), (52) and (53).

Claim 18 (Asymptotic expansions III, case $m=2$ ). The following identity holds

$$
\left.\partial_{\nu} d_{0, \nu}\right|_{\nu=0}=-\left[\frac{(p-3)(2 p-1)\left(24-23 p+3 p^{2}+2 p^{3}\right)}{36\left(p^{2}-1\right)(p-2)}\right] \int_{\mathbb{R}}\left(Q^{0}\right)^{p},
$$

for all $p \geq 3$.

Proof. The proof of the above result is equivalent to prove that for the nonlinearity $f_{0, \nu}(s)=$ $s^{2}+\mu s^{p}, p \geq 3$ and $\nu$ small, we have

$$
d_{0, \nu}=b_{2,0}\left(f_{0, \nu}\right)+\frac{1}{6} b_{1,0}^{3}\left(f_{0, \nu}\right)=-\nu\left[\frac{(p-3)(2 p-1)\left(24-23 p+3 p^{2}+2 p^{3}\right)}{36\left(p^{2}-1\right)(p-2)}\right] \int_{\mathbb{R}}\left(Q^{0}\right)^{p}+o(\nu) .
$$

First of all, note that we can expand $b_{2,0}=b_{2,0}^{0}+\nu b_{2,0}^{1}+o(\nu)$, with $b_{2,0}^{0}=\frac{4}{3}$ (cf. [24], Lemma 3.1). By considering (143) in Claim 11. Claim 16 and expanding at first order in $\nu$, we get

$$
\begin{aligned}
b_{2,0}^{1}= & -8 b_{1,0}^{1}+\frac{1}{4} p(p-1) \int_{\mathbb{R}}\left(Q^{0}\right)^{p-2}\left(1+A_{1,0}^{0}\right)^{3}+\frac{1}{2} \int_{\mathbb{R}} A_{1,0}^{1}\left(1+3\left(A_{1,0}^{0}\right)^{2}\right)-\frac{1}{2} a_{1,0}^{1} \int_{\mathbb{R}} Q^{0} A_{1,0}^{0} \\
& -\frac{1}{3} \int_{\mathbb{R}} Q^{1} A_{1,0}^{0}-\frac{1}{3} \int_{\mathbb{R}} Q^{0} A_{1,0}^{1}-a_{1,0}^{1} \int_{\mathbb{R}}\left(Q^{0}\right)^{\prime 2}-\frac{4}{9} \int_{\mathbb{R}}\left(Q^{0}\right)^{\prime}\left(Q^{1}\right)^{\prime} \\
& +\frac{2}{3} a_{1,0}^{1} \int_{\mathbb{R}}\left(Q^{0}\right)^{2}\left(1-2\left(1+A_{1,0}^{0}\right)\right)+\frac{2}{9} \int_{\mathbb{R}} Q^{0} Q^{1}\left(1-2\left(1+A_{1,0}^{0}\right)\right) \\
& +\frac{2}{9} \int_{\mathbb{R}} Q^{0}\left[Q^{1}-2 Q^{0} A_{1,0}^{1}-\left(2 Q^{1}+p\left(Q^{0}\right)^{p-1}\right)\left(1+A_{1,0}^{0}\right)\right] \\
& -\frac{3}{4} a_{1,0}^{1} \int_{\mathbb{R}}\left[2 Q^{0}\left(1+A_{1,0}^{0}\right)+3\left(A_{1,0}^{0}\right)^{\prime \prime}\right] A_{1,0}^{0}-\frac{1}{2} \int_{\mathbb{R}}\left[2 Q^{0}\left(1+A_{1,0}^{0}\right)+3\left(A_{1,0}^{0}\right)^{\prime \prime}\right] A_{1,0}^{1} \\
& -\frac{1}{2} \int_{\mathbb{R}}\left[\left(2 Q^{1}+p\left(Q^{0}\right)^{p-1}\right)\left(1+A_{1,0}^{0}\right)+2 Q^{0} A_{1,0}^{1}+3\left(A_{1,0}^{1}\right)^{\prime \prime}\right] A_{1,0}^{0} \\
& +\frac{1}{2} \int_{\mathbb{R}} B_{1,0}^{0}\left(2 Q^{1}+p\left(Q^{0}\right)^{p-1}\right) \int_{0}^{x}\left(A_{1,0}^{0}+\frac{2}{3} Q^{0}\right)+\frac{1}{2} \int_{\mathbb{R}} B_{1,0}^{0}\left[3\left(A_{1,0}^{1}\right)^{\prime}\right. \\
& \left.+2 Q^{0} \int_{0}^{x}\left(A_{1,0}^{1}+a_{1,0}^{1} Q^{0}+\frac{2}{3} Q^{1}\right)\right]+4 a_{1,0}^{1} \int_{\mathbb{R}}\left(Q^{0}\right)^{\prime \prime} A_{1,0}^{0}+\frac{4}{3} \int_{\mathbb{R}}\left(Q^{1}\right)^{\prime \prime} A_{1,0}^{0}+\frac{4}{3} \int_{\mathbb{R}}\left(Q^{0}\right)^{\prime \prime} A_{1,0}^{1} .
\end{aligned}
$$


Now we arrange the above expression according to $a_{1,0}^{1}, A_{1,0}^{1}, Q^{1}, b_{1,0}^{1}, B_{1,0}^{1}$ and the rest terms. We obtain,

$$
\begin{aligned}
b_{2,0}^{1}= & -8 b_{1,0}^{1}+\frac{1}{4} p(p-1) \int_{\mathbb{R}}\left(Q^{0}\right)^{p-2}\left(1+A_{1,0}^{0}\right)^{3}-\frac{p}{3} \int_{\mathbb{R}}\left(Q^{0}\right)^{p-1}\left(1+A_{1,0}^{0}\right)\left(\frac{3}{2} A_{1,0}^{0}+\frac{2}{3} Q^{0}\right) \\
& +p \int_{\mathbb{R}} B_{1,0}^{0}\left(Q^{0}\right)^{p-2}\left(Q^{0}\right)^{\prime}+\int_{\mathbb{R}} B_{1,0}^{0} Q^{0} \int_{0}^{x} A_{1,0}^{1}+\frac{1}{2} \int_{\mathbb{R}} A_{1,0}^{1} F_{A}+\delta a_{1,0}^{1}+\int_{\mathbb{R}} Q^{1} F_{Q} \\
& +\frac{2}{3} \int_{\mathbb{R}} B_{1,0}^{0} Q^{0} \int_{0}^{x} Q^{1},
\end{aligned}
$$

where

$$
\begin{aligned}
& F_{A}:=1+3\left(A_{1,0}^{0}\right)^{2}- \frac{2}{3} Q^{0}-\frac{8}{9}\left(Q^{0}\right)^{2}-\left(2 Q^{0}+2 Q^{0} A_{1,0}^{0}+3\left(A_{1,0}^{0}\right)^{\prime \prime}\right)-\left(2 Q^{0} A_{1,0}^{0}+3\left(A_{1,0}^{0}\right)^{\prime \prime}\right) \\
&-3\left(B_{1,0}^{0}\right)^{\prime}+ \frac{8}{3}\left(Q^{0}\right)^{\prime \prime} ; \\
& \delta:=-\frac{1}{2} \int_{\mathbb{R}} Q^{0} A_{1,0}^{0}+\int_{\mathbb{R}} Q^{0}\left(Q^{0}\right)^{\prime \prime}-\frac{2}{3} \int_{\mathbb{R}}\left(Q^{0}\right)^{2}\left(1+2 A_{1,0}^{0}\right) \\
& \quad-\frac{3}{4} \int_{\mathbb{R}}\left(2 Q^{0}+2 Q^{0} A_{1,0}^{0}+3\left(A_{1,0}^{0}\right)^{\prime \prime}\right) A_{1,0}^{0}+4 \int_{\mathbb{R}}\left(Q^{0}\right)^{\prime \prime} A_{1,0}^{0} ;
\end{aligned}
$$

and

$$
F_{Q}:=-\frac{1}{3} A_{1,0}^{0}+\frac{4}{9}\left(Q^{0}\right)^{\prime \prime}-\frac{4}{9} Q^{0}\left(1+2 A_{1,0}^{0}\right)-\left(1+A_{1,0}^{0}\right) A_{1,0}^{0}-2 \varphi^{0} B_{1,0}^{0}+\frac{4}{3}\left(A_{1,0}^{0}\right)^{\prime \prime} .
$$

Note that we have used that

$$
\int_{\mathbb{R}} B_{1,0}^{0}\left(Q^{0}\right)^{\prime}=0, \quad \int_{0}^{x}\left(A_{1,0}^{0}+\frac{2}{3} Q^{0}\right)=-2 \varphi^{0} .
$$

Now we use the expressions (183) and (184) in Claim 17 to have

$$
\begin{aligned}
b_{2,0}^{1}+2 b_{1,0}^{1}= & -\frac{12}{5} a_{1,0}^{1}+\frac{1}{4} p(p-1) \int_{\mathbb{R}}\left(Q^{0}\right)^{p-2}\left(1-\frac{4}{3} Q^{0}\right)^{3}+\frac{4 p}{9} \int_{\mathbb{R}}\left(Q^{0}\right)^{p}\left(1-\frac{4}{3} Q^{0}\right) \\
& +p \int_{\mathbb{R}}\left(Q^{0}\right)^{p-1}\left[2-\frac{14}{3} Q^{0}+\frac{20}{9}\left(Q^{0}\right)^{2}\right]+\frac{1}{2} \int_{\mathbb{R}} A_{1,0}^{1}\left[-5+16 Q^{0}-\frac{68}{9}\left(Q^{0}\right)^{2}\right] \\
& +\int_{\mathbb{R}} Q^{1}\left[2-\frac{32}{3} Q^{0}+\frac{170}{27}\left(Q^{0}\right)^{2}\right] .
\end{aligned}
$$

Note that we have also made use of (171) with $\tilde{\mu}=0$ to obtain

$$
\begin{aligned}
-3 \int_{\mathbb{R}} Q^{0}-\frac{1}{2} \int_{\mathbb{R}} Q^{0} A_{1,0}^{0}+\int_{\mathbb{R}} Q^{0}\left(Q^{0}\right)^{\prime \prime}-\frac{2}{3} \int_{\mathbb{R}}\left(Q^{0}\right)^{2}\left(1+2 A_{1,0}^{0}\right) \\
\quad-\frac{3}{4} \int_{\mathbb{R}}\left(2 Q^{0}+2 Q^{0} A_{1,0}^{0}+3\left(A_{1,0}^{0}\right)^{\prime \prime}\right) A_{1,0}^{0}+4 \int_{\mathbb{R}}\left(Q^{0}\right)^{\prime \prime} A_{1,0}^{0}=-\frac{12}{5} .
\end{aligned}
$$

Using (179), (180) and (182), we have

$$
\begin{aligned}
& b_{2,0}^{1}+2 b_{1,0}^{1}=-\frac{12}{5} a_{1,0}^{1}+\frac{1}{4} p(p-1) \int_{\mathbb{R}}\left(Q^{0}\right)^{p-2}\left(1-\frac{4}{3} Q^{0}\right)^{3}+\frac{4 p}{9} \int_{\mathbb{R}}\left(Q^{0}\right)^{p}\left(1-\frac{4}{3} Q^{0}\right) \\
& +\frac{1}{2} \int_{\mathbb{R}}\left[-a_{1,0}^{1}\left(3 Q^{0}-2\left(Q^{0}\right)^{2}\right)+p\left(Q^{0}\right)^{p-1}-\frac{4}{3}(p-1)\left(Q^{0}\right)^{p}\right]\left[-5-6 \Lambda Q^{0}+\frac{68}{9} Q^{0}\right] \\
& \quad+\int_{\mathbb{R}}\left(Q^{0}\right)^{p}\left[2+\frac{20}{3} \Lambda Q^{0}-\frac{170}{27} Q^{0}\right]+p \int_{\mathbb{R}}\left(Q^{0}\right)^{p-1}\left[2-\frac{14}{3} Q^{0}+\frac{20}{9}\left(Q^{0}\right)^{2}\right] .
\end{aligned}
$$

A simple computation using (175) and (171) with $\tilde{\mu}=0$ shows that

$$
\int_{\mathbb{R}}\left(3 Q^{0}-2\left(Q^{0}\right)^{2}\right)\left(-5-6 \Lambda Q^{0}+\frac{68}{9} Q^{0}\right)=-\frac{59}{5} .
$$


Thus, replacing the value of $a_{1,0}^{1}$ given by (183),

$$
\begin{aligned}
& b_{2,0}^{1}+2 b_{1,0}^{1}=-\frac{7}{18}\left[\frac{(p-3)(2 p-1)}{p+1}\right] \int_{\mathbb{R}}\left(Q^{0}\right)^{p}+\frac{4 p}{9} \int_{\mathbb{R}}\left(Q^{0}\right)^{p}\left(1-\frac{4}{3} Q^{0}\right) \\
& +\frac{1}{4} p(p-1) \int_{\mathbb{R}}\left(Q^{0}\right)^{p-2}\left(1-\frac{4}{3} Q^{0}\right)^{3}+p \int_{\mathbb{R}}\left(Q^{0}\right)^{p-1}\left[2-\frac{14}{3} Q^{0}+\frac{20}{9}\left(Q^{0}\right)^{2}\right] \\
& +\frac{1}{2} \int_{\mathbb{R}}\left[p\left(Q^{0}\right)^{p-1}-\frac{4}{3}(p-1)\left(Q^{0}\right)^{p}\right]\left[-5-6 \Lambda Q^{0}+\frac{68}{9} Q^{0}\right] \\
& +2 \int_{\mathbb{R}}\left(Q^{0}\right)^{p}+\frac{10}{3} \frac{2 p+1}{p+1} \int_{\mathbb{R}}\left(Q^{0}\right)^{p+1}-\frac{170}{27} \int_{\mathbb{R}}\left(Q^{0}\right)^{p+1} .
\end{aligned}
$$

Simplifiying, we get

$$
\begin{aligned}
& b_{2,0}^{1}+2 b_{1,0}^{1}=-\frac{7}{18} \frac{(p-3)(2 p-1)}{p+1} \int_{\mathbb{R}}\left(Q^{0}\right)^{p}+\frac{1}{4} p(p-1) \int_{\mathbb{R}}\left(Q^{0}\right)^{p-2}-p\left(p-\frac{1}{2}\right) \int_{\mathbb{R}}\left(Q^{0}\right)^{p-1} \\
& +\left(\frac{1}{6}-\frac{13}{9} p+\frac{4}{3} p^{2}\right) \int_{\mathbb{R}}\left(Q^{0}\right)^{p}+\left(-\frac{16}{27}+\frac{32}{27} p+\frac{2}{3} \frac{1}{p+1}-\frac{16}{27} p^{2}\right) \int_{\mathbb{R}}\left(Q^{0}\right)^{p+1} .
\end{aligned}
$$

Using (171) with $\tilde{\mu}=0$ and the fact that $p \geq 3$, we finally obtain

$$
\begin{aligned}
b_{2,0}^{1}+2 b_{1,0}^{1}= & -\frac{7}{18} \frac{(p-3)(2 p-1)}{p+1}+\frac{1}{36} p \frac{(2 p-1)(2 p-3)}{p-2}-\frac{1}{3} p\left(p-\frac{1}{2}\right) \frac{(2 p-1)}{p-1}+ \\
& \left.+\left(\frac{1}{6}-\frac{13}{9} p+\frac{4}{3} p^{2}\right)+\frac{3 p}{1+2 p}\left(-\frac{16}{27}+\frac{32}{27} p+\frac{2}{3} \frac{1}{p+1}-\frac{16}{27} p^{2}\right)\right] \int_{\mathbb{R}}\left(Q^{0}\right)^{p} \\
= & -\frac{(p-3)(2 p-1)\left(24-23 p+3 p^{2}+2 p^{3}\right)}{36\left(p^{2}-1\right)(p-2)} \int_{\mathbb{R}}\left(Q^{0}\right)^{p} .
\end{aligned}
$$

Let us define, for $p$ real, $f(p):=24-23 p+3 p^{2}+2 p^{3}$. Then we have

$$
f(p) \geq 36 \text { for all } p \geq 3 .
$$

It is clear that this last affirmation allows us to conclude the proof. Let us prove (186). Note that $f(3)=36$ and $f^{\prime}(p)$ is given by $f^{\prime}(p)=6 p^{2}+6 p-23>0$ for all $p \geq 3$. This implies (186). The proof is complete.

Remark B.3. First of all, note that in the above expression we recover the integrability condition of the Gardner equation $(p=3)$. Furthermore, note that this term is divergent when we formally take the limit $p \downarrow 2$ and the equation approaches the integrable case. This can be explained by the higher regularity needed in our results $\left(f \in C^{4}\right.$ for $\left.m=2\right)$, to justify the asymptotics. Indeed, from (52) and (143) we need at least $f \in C^{3}(\mathbb{R})$, and $f(s):=s^{2}+\varepsilon s^{p}$ is not $C^{3}$ at zero as $p \downarrow 2$, $p>2$. In addition, the terms in (52), (53)

$$
\frac{1}{2}\left(f^{\prime \prime}(Q)-2\right)^{\prime}, \frac{1}{2}\left(f^{\prime \prime}(Q)-2\right),
$$

vanish in the integrable case $m=p=2$. For the computation in the quadratic case, see Proposition 2.1 and Lemma 3.1 in 24 .

Acknowledgments. The author would like to thank Yvan Martel and Frank Merle for their continuous encouragement in the elaboration of this work. Part of this work was written at DIM, Universidad de Chile. The author thanks the DIM members for their warm hospitality. Finally, the author wishes to thank J. Colliander for pointing him out the case of the Gardner nonlinearity.

\section{REFERENCES}

[1] Ablowitz, M. J. and Clarkson, P. A., Solitons, nonlinear evolution equations and inverse scattering. London Mathematical Society Lecture Note Series, 149. Cambridge University Press, Cambridge, 1991.

[2] M. J. Ablowitz, D. J. Kaup, A. C. Newell, and H. Segur, The inverse scattering transform - Fourier analysis for non linear problems, Stud. Appl. Math. 53, (1974), 249-315.

[3] W. K. Abou Salem, J. Fröhlich, and I . M. Sigal, Colliding solitons for the nonlinear Schrödinger equation, to appear in Comm. Math. Phys. 
[4] H. Berestycki and P.-L. Lions, Nonlinear scalar field equations. I. Existence of a ground state, Arch. Rational Mech. Anal. 82, (1983) 313-345.

[5] J.L. Bona, W.G. Pritchard and L.R. Scott, Solitary-wave interaction, Phys. Fluids 23, 438, (1980).

[6] J.L. Bona, P. Souganidis and W. Strauss, Stability and instability of solitary waves of Korteweg-de Vries type, Proc. Roy. Soc. London 411 (1987), 395-412.

[7] E. Fermi, J. Pasta and S. Ulam, Studies of nonlinear problems I, Los Alamos Report LA1940 (1955); reproduced in Nonlinear Wave Motion, A.C. Newell, ed., Am. Math. Soc., Providence, R. I., 1974, pp. 143-156.

[8] C.S. Gardner, M.D. Kruskal, and R. Miura, Korteweg-de Vries equation and generalizations. II. Existence of conservation laws and constants of motion, J. Math. Phys. 9, no. 8 (1968), 1204-1209.

[9] M. Hărăguş-Courcelle and D.H. Sattinger, Inversion of the linearized Korteweg-de Vries equation at the multisoliton solutions, Z. Angew. Math. Phys. 49 (1998), 436-469.

[10] R. Hirota, Exact solution of the Korteweg-de Vries equation for multiple collisions of solitons, Phys. Rev. Lett., 27 (1971), 1192-1194.

[11] J. Holmer, and M. Zworski, Soliton interaction with slowly varying potentials, Int. Math. Res. Not., (2008) 2008, art. ID rnn026, 36 pp.

[12] J. Holmer, J. Marzuola, and M. Zworski, Soliton Splitting by External Delta Potentials, J. Nonlinear Sci. 17 no. 4 (2007), 349-367.

[13] J. Holmer, J. Marzuola and M. Zworski, Fast soliton scattering by delta impurities, Comm. Math. Phys., 274, no.1 (2007) 187-216.

[14] H. Kalisch and J.L. Bona, Models for internal waves in deep water, Disc. Cont. Dyn. Syst., 6 (2000), 1-20.

[15] D.J. Korteweg and G. de Vries, On the change of form of long waves advancing in a rectangular canal, and on a new type of stationary waves, Philos. Mag. Ser. 5, 39 (1895), 422-443.

[16] C.E. Kenig, G. Ponce and L. Vega, Well-posedness and scattering results for the generalized Korteweg-de Vries equation via the contraction principle, Comm. Pure Appl. Math. 46, (1993) 527-620.

[17] P. D. Lax, Integrals of nonlinear equations of evolution and solitary waves, Comm. Pure Appl. Math. 21, (1968) 467-490.

[18] Yi Li and D.H. Sattinger, Soliton collisions in the ion acoustic plasma equations, J. Math. Fluid Mech. 1 (1999), 117-130.

[19] Y. Martel, Asymptotic N-soliton-like solutions of the subcritical and critical generalized Korteweg-de Vries equations, Amer. J. Math. 127 (2005), 1103-1140.

[20] Y. Martel, Linear problems related to asymptotic stability of solitons of the generalized KdV equations, SIAM J. Math. Anal. 38 (2006), 759-781.

[21] Y. Martel and F. Merle, Blow up in finite time and dynamics of blow up solutions for the $L^{2}$-critical generalized KdV equation, J. Amer. Math. Soc. 15 (2002), no. 3, 617-664 (electronic).

[22] Y. Martel and F. Merle, Asymptotic stability of solitons of the gKdV equations with general nonlinearity, Math. Ann. 34 (2008), no. 2, 391-427.

[23] Y. Martel and F. Merle, Refined asymptotics around solitons for gKdV equations, Discrete contin. Dyn. Syst. 20 (2008), no. 2, 177-218.

[24] Y. Martel and F. Merle, Description of two soliton collision for the quartic gKdV equations, preprint.

[25] Y. Martel and F. Merle, Stability of two soliton collision for nonintegrable gKdV equations, Comm. Math. Phys. 286 (2009), 39-79.

[26] Y. Martel, F. Merle and T. Mizumachi, Description of the inelastic collision of two solitary waves for the $B B M$ equation, to appear in Arch. Rat. Mech. Anal.

[27] R.M. Miura, The Korteweg-de Vries equation: a survey of results, SIAM Review 18, (1976) 412-459.

[28] T. Mizumachi, Weak interaction between solitary waves of the generalized KdV equations, SIAM J. Math. Anal. 35 (2003), 1042-1080.

[29] G.S. Perelman, Asymptotic stability of multi-soliton solutions for nonlinear Schrdinger equations. Comm. Partial Differential Equations 29, (2004) 1051-1095.

[30] L.Y. Shih, Soliton-like interaction governed by the generalized Korteweg-de Vries equation, Wave motion 2 (1980), 197-206.

[31] P. C. Schuur, Asymptotic analysis of solitons problems, Lect. Notes in Math. 1232 (1986), Springer-Verlag, Berlin.

[32] A.-M. Wazwaz, New soliton solutions for the gardner equation, Comm. Nonlinear Sc. Num. Sim. 12, (2007) 1395-1404.

[33] M.I. Weinstein, Lyapunov stability of ground states of nonlinear dispersive evolution equations, Comm. Pure Appl. Math. 39, (1986) 51-68.

[34] N.J. Zabusky and M.D. Kruskal, Interaction of "solitons" in a collisionless plasma and recurrence of initial states, Phys. Rev. Lett. 15 (1965), 240-243.

Université de Versailles Saint-Quentin-en-Yvelines, LMV-UMR 8100, 45 AV. Des Etats-Unis, 78035 Versailles cedex, France

E-mail address: Claudio.Munoz@math.uvsq.fr 